\title{
Precise Amphiphilic Giant Polymeric Chain Based on Nanosized Monomers with Exact Regio-Configuration
}

Zhongguo Liu, ${ }^{1}$ Xin Chen, ${ }^{1}$ Ze Yang, ${ }^{1}$ Shuai Wang, ${ }^{1}$ Zhanhui Gan, ${ }^{1}$ Gang Li,${ }^{4}$ and Xue-Hui Dong ${ }^{1,2,3 *}$

${ }^{1}$ South China Advanced Institute for Soft Matter Science and Technology, School of Molecular Science and Engineering, South China University of Technology, Guangzhou 510640, China

${ }^{2}$ Guangdong Provincial Key Laboratory of Functional and Intelligent Hybrid Materials and Devices, South China University of Technology, Guangzhou 510640, China

${ }^{3}$ State Key Laboratory of Luminescent Materials and Devices, South China University of Technology, Guangzhou 510640, China

${ }^{4}$ Department of Ecology and Environment, Yuzhang Normal University, Nanchang 330103, China

*Corresponding author: $\underline{x d o n g @ s c u t . e d u . c n}$ 


\section{Chemicals and Solvents}

The following chemicals were used as received: octavinylPOSS (Hybrid Plastics), 3-mercapto-1-propanol (Sigma, 95\%), 6-mercapto-1-hexanol (Adamas, 98\%), 1-butanethiol (Sigma, 93\%), maleimide (Sigma, 95\%), furan (Sigma, 98\%), 1-thioglycerol (Sigma, 98\%), 2,2-dimethoxy-2-phenylacetophenone (DMPA, 98\%, TCI America), diisopropyl azodicarboxylate (DIAD, 97\%, TCI America), triphenylphosphine $\left(\mathrm{PPh}_{3}\right.$, 97\%, TCI America), tetrabromomethane $\left(\mathrm{CBr}_{4}, 98 \%\right.$, TCI America), potassium thioacetate (KSAc, 98\%, TCI America), acetyl chloride (98\%, TCI America), anhydrous sodium sulfate $\left(\mathrm{Na}_{2} \mathrm{SO}_{4}\right.$, Sigma, $\left.93 \%\right)$, tetrahydrofuran (THF), triethylamine (TEA), toluene, petroleum ether (PE), ethyl acetate (EA), methanol $(\mathrm{MeOH})$, chloroform $\left(\mathrm{CHCl}_{3}\right)$, dichloromethane $\left(\mathrm{CH}_{2} \mathrm{Cl}_{2}\right)$.

\section{Characterizations}

Nuclear Magnetic Resonance (NMR). All ${ }^{1} \mathrm{H}$ NMR spectra were acquired in $\mathrm{CDCl}_{3}$ using a Bruker 500 MHz NMR spectrometer. The spectra were referenced to the residual proton impurities in the $\mathrm{CDCl}_{3}$ at $\delta$ 7.27 ppm. ${ }^{29} \mathrm{Si}$ NMR spectra were acquired in $\mathrm{CDCl}_{3}$ using a Bruker $99 \mathrm{MHz} \mathrm{NMR}$ spectrometer and referenced to tetramethylsilane (TMS) at $\delta 0.00 \mathrm{ppm}$.

Size exclusion chromatography (SEC). SEC analyses for polymeric chains were measured at $35{ }^{\circ} \mathrm{C}$ on a Tosoh HLC-8320 instrument equipped with three TSKgel columns (SuperH2000, SuperH3000, and SuperH4000) in series, a double flow type RI detector, and a UV-8320 UV detector. The eluting solvent is THF. The flow rate was $0.6 \mathrm{~mL} / \mathrm{min}$. Data acquisition was performed using EcoSEC software. And PS was used as the standard for molecular weight calculation.

Matrix-assisted laser desorption/ionization time-of-flight (MALDI-ToF). All MALDI-TOF mass spectra were obtained on an UltrafleXtreme MALDI ToF mass spectrometer (Bruker Daltonics) equipped with a 1 kHz smart beam-II laser. The matrix trans-2-[3-(4-tert-butyl-phenyl)-2-methyl-2-propenylidene]-malononitrile (DCTB, Aldrich, $>98 \%)$ was prepared in $\mathrm{CHCl}_{3}(20 \mathrm{mg} / \mathrm{mL})$. The cationizing agent sodium trifluoroacetate was prepared in ethanol $(10 \mathrm{mg} / \mathrm{mL})$. The matrix and cationizing salt solutions were mixed in a ratio of $10 / 1(\mathrm{v} / \mathrm{v})$. All samples were dissolved in $\mathrm{CHCl}_{3}$ at a concentration of $10 \mathrm{mg} / \mathrm{mL}$. The attenuation of the laser was adjusted to minimize undesired polymer fragmentation and to maximize the sensitivity.

Small angle X-ray scattering (SAXS). SAXS experiments were performed on beamline BL16B1 at Shanghai Synchrotron Radiation (SSRF). The incident X-ray photon energy was $10 \mathrm{keV}$; the wavelength of the X-ray was $0.124 \mathrm{~nm}$; the photo flux was $1 \times 10^{11} \mathrm{phs} / \mathrm{s}$. The beam size is smaller than $0.4 \times 0.5 \mathrm{~mm}^{2}$. 
Scattered X-rays were captured on a 2-dimensional Pilatus detector. The instrument was calibrated with diffraction patterns from silver behenate.

Recycling preparative SEC. Sample separation were performed on a LaboACE LC-5060 (Japan Analytical Industry Co., Ltd) instrument equipped with two JAIGEL-HR columns (2HR and 2.5HR) in series, a double flow type RI-700 LA detector, and a UV-4ch 800 LA detector. THF was used as eluent with a flow-rate of 10 $\mathrm{mL} / \mathrm{min}$. Crude polymers were dissolved in THF at a concentration of $50 \mathrm{mg} / \mathrm{mL}$ and filtered through a 0.45 $\mu \mathrm{m}$ PTFE syringe filter prior to inject. The data were monitored in real time by JAI scan software and the target fraction was collected manually.

Differential Scanning Calorimetry (DSC). Thermal transitions of all the samples were characterized utilizing a PerkinElmer PYRIS Diamond DSC with an Intracooler 2P apparatus. The samples were sealed in a DSC pan with an initial mass of about 5-8 $\mathrm{mg}$ and scanned from -60 to $80{ }^{\circ} \mathrm{C}$ with a heating/cooling rate of $5{ }^{\circ} \mathrm{C} / \mathrm{min}$ under nitrogen flow.

Thermogravimetric Analysis (TGA). Nonisothermal decomposition experiments were performed on a thermogravimetric analysis instrument (Model Q500, TA Instruments) under nitrogen atmosphere with a heating rate of $10^{\circ} \mathrm{C} / \mathrm{min}$. 


\section{Syntheses}

\subsection{CPOSS and *CPOSS monomer}

The syntheses of CPOSS monomer and ${ }^{*}$ CPOSS monomer follow the same protocol except different thiol ligands are used in the first step (Scheme S1).

a) para-, meta-, and ortho-VPOSS(OH)2 and *VPOSS(OH)2. OctavinylPOSS (VPOSS, 30.0 g, 47.4 mmol), thiol ligands (3-mercapto-1-propanol: $8.72 \mathrm{~g}, 94.8 \mathrm{mmol}$; or 6-mercapto-1-hexanol: $12.7 \mathrm{~g}, 94.8$ mmol), and DMPA (140 mg, $0.55 \mathrm{mmol})$ were mixed and fully dissolved in $350 \mathrm{~mL}$ of THF. After irradiation with UV $365 \mathrm{~nm}$ for $30 \mathrm{~min}$, the mixture was purified by flash column chromatography on silica gel using EA/DCM (v/v from 1/20 to $1 / 4)$ as the eluent to afford the products as white powders.

para-VPOSS(OH)2 (1.2 g, yield: 3.1\%). ${ }^{1} \mathrm{H}$ NMR (500 MHz, $\left.\mathrm{CDCl}_{3}, \mathrm{ppm}, \delta\right): 6.15-5.87(\mathrm{~m}, 18 \mathrm{H}$, $\left.-\mathrm{C} \underline{\mathrm{H}}=\mathrm{C}_{2}\right), 3.76\left(\mathrm{t}, J=5.9 \mathrm{~Hz}, 4 \mathrm{H},-\underline{\mathrm{C}}_{2} \mathrm{OH}\right), 2.69-2.65\left(\mathrm{~m}, 8 \mathrm{H},-\mathrm{CH}_{2} \mathrm{C}_{2} \mathrm{SC}_{2} \mathrm{CH}_{2}-\right), 1.85$ (tt, $J=7.1,6.1$ $\left.\mathrm{Hz}, 4 \mathrm{H},-\mathrm{SCH}_{2} \underline{\mathrm{C}}_{2} \mathrm{CH}_{2} \mathrm{OH}\right), 1.07$ (m, 4H, $\left.-\mathrm{SiC}_{2}{ }^{-}\right) .{ }^{29} \mathrm{Si} \mathrm{NMR}\left(99 \mathrm{MHz}, \mathrm{CDCl}_{3}, \mathrm{ppm}, \delta\right.$ ): -68.53, -80.35.

meta-VPOSS(OH) 2 (2.8 g, yield: 7.2\%). ${ }^{1} \mathrm{H}$ NMR (500 MHz, $\left.\mathrm{CDCl}_{3}, \mathrm{ppm}, \delta\right): 6.15-5.86(\mathrm{~m}, 18 \mathrm{H}$, $\left.-\mathrm{C} \underline{\mathrm{H}}=\mathrm{C}_{2}\right), 3.76\left(\mathrm{t}, J=5.5 \mathrm{~Hz}, 4 \mathrm{H},-\underline{\mathrm{CH}}_{2} \mathrm{OH}\right), 2.69-2.66\left(\mathrm{~m}, 8 \mathrm{H},-\mathrm{CH}_{2} \mathrm{CH}_{2} \mathrm{SC}_{2} \mathrm{CH}_{2}-\right), 1.84(\mathrm{tt}, J=7.1,6.1$ $\left.\mathrm{Hz}, 4 \mathrm{H},-\mathrm{SCH}_{2} \underline{\mathrm{C}}_{2} \mathrm{CH}_{2} \mathrm{OH}\right), 1.07$ (m, 4H, - $\left.\mathrm{SiC}_{2}-\right) .{ }^{29} \mathrm{Si} \mathrm{NMR}$ (99 MHz, $\mathrm{CDCl}_{3}, \mathrm{ppm}, \delta$ ): -68.52, -80.23, $-80.35,-80.48$.

ortho-VPOSS(OH) 2 (3.5 g, yield: 9.1\%). ${ }^{1} \mathrm{H}$ NMR (500 MHz, $\left.\mathrm{CDCl}_{3}, \mathrm{ppm}, \delta\right)$ : 6.14-5.87 (m, 18H, $\left.-\mathrm{C} \underline{\mathrm{H}}=\mathrm{C}_{2}\right), 3.76\left(\mathrm{t}, J=6.0 \mathrm{~Hz}, 4 \mathrm{H},-\underline{\mathrm{C}}_{2} \mathrm{OH}\right), 2.69-2.64\left(\mathrm{~m}, 8 \mathrm{H},-\mathrm{CH}_{2} \mathrm{C}_{2}{ }_{2} \mathrm{SC}_{2}{ }_{2} \mathrm{CH}_{2}-\right), 1.85(\mathrm{tt}, J=7.1,6.1$ $\left.\mathrm{Hz}, 4 \mathrm{H},-\mathrm{SCH}_{2} \mathrm{CH}_{2} \mathrm{CH}_{2} \mathrm{OH}\right), 1.08$ (m, 4H, $\left.-\mathrm{SiCH}_{2}-\right) .{ }^{29} \mathrm{Si} \mathrm{NMR}$ (99 MHz, $\mathrm{CDCl}_{3}, \mathrm{ppm}, \delta$ ): -68.63, -80.22, -80.36 .

para-*VPOSS(OH)2 $(1.0$ g, yield: $2.3 \%) .{ }^{1} \mathrm{H}$ NMR $\left(500 \mathrm{MHz}, \mathrm{CDCl}_{3}, \mathrm{ppm}, \delta\right): 6.15-5.88(\mathrm{~m}, 18 \mathrm{H}$, $\left.-\mathrm{C} \underline{\mathrm{H}}=\mathrm{C}_{2}\right), 3.65\left(\mathrm{~s}, J=6.0 \mathrm{~Hz}, 4 \mathrm{H},-\underline{\mathrm{C}}_{2} \mathrm{OH}\right), 2.64\left(\mathrm{~s}, 4 \mathrm{H},-\mathrm{SiCH}_{2} \mathrm{CH}_{2} \mathrm{SC}_{2} \underline{\mathrm{H}}_{2}\right), 2.53 \quad(\mathrm{~s}, 4 \mathrm{H}$, $\left.-\mathrm{SiCH}_{2} \mathrm{C}_{2} \mathrm{SCH}_{2}-\right), 1.61\left(\mathrm{~s}, 8 \mathrm{H},-\underline{\mathrm{CH}}_{2} \mathrm{C}_{2} \mathrm{CH}_{2} \mathrm{OH}\right), 1.41\left(\mathrm{~s}, 8 \mathrm{H},-\underline{\mathrm{C}}_{2} \underline{\mathrm{C}}_{2} \mathrm{CH}_{2} \mathrm{CH}_{2} \mathrm{CH}_{2} \mathrm{OH}\right), 1.09$ (s, 4H, $\left.-\mathrm{SiCH}_{2}-\right)$.

meta-*VPOSS(OH)2 $(2.1$ g, yield: $4.9 \%) .{ }^{1} \mathrm{H}$ NMR (500 MHz, $\left.\mathrm{CDCl}_{3}, \mathrm{ppm}, \delta\right): 6.18-5.84(\mathrm{~m}, 18 \mathrm{H}$, $\left.-\mathrm{C} \underline{\mathrm{H}}=\mathrm{C}_{2}\right), 3.66\left(\mathrm{~s}, J=6.1 \mathrm{~Hz}, 4 \mathrm{H},-\mathrm{CH}_{2} \mathrm{OH}\right), 2.64 \quad\left(\mathrm{~s}, 4 \mathrm{H},-\mathrm{SiCH}_{2} \mathrm{CH}_{2} \mathrm{SC}_{2}-\right), 2.52 \quad(\mathrm{~s}, 4 \mathrm{H}$, $\left.-\mathrm{SiCH}_{2} \mathrm{CH}_{2} \mathrm{SCH}_{2}-\right), 1.60$ (s, 8H, - $\left.\underline{\mathrm{CH}}_{2} \mathrm{C}_{2} \mathrm{CH}_{2} \mathrm{OH}\right), 1.40\left(\mathrm{~s}, 8 \mathrm{H},-\underline{\mathrm{C}}_{2} \mathrm{C}_{2} \mathrm{CH}_{2} \mathrm{CH}_{2} \mathrm{CH}_{2} \mathrm{OH}\right), 1.08$ (s, 4H, $\left.-\mathrm{SiC}_{2}-\right)$.

ortho-*VPOSS(OH) 2 (2.8 g, yield: $6.6 \%) .{ }^{1} \mathrm{H}$ NMR (500 MHz, $\left.\mathrm{CDCl}_{3}, \mathrm{ppm}, \delta\right): 6.15-5.88(\mathrm{~m}, 18 \mathrm{H}$, $\left.-\mathrm{CH}=\underline{\mathrm{CH}}_{2}\right), 3.62\left(\mathrm{~s}, J=6.2 \mathrm{~Hz}, 4 \mathrm{H},-\underline{\mathrm{CH}}_{2} \mathrm{OH}\right), 2.67 \quad\left(\mathrm{~s}, 4 \mathrm{H},-\mathrm{SiCH}_{2} \mathrm{CH}_{2} \mathrm{SC}_{2}-\underline{H}_{2}\right), 2.55 \quad(\mathrm{~s}, 4 \mathrm{H}$, 
$\left.-\mathrm{SiCH}_{2} \mathrm{CH}_{2} \mathrm{SCH}_{2}-\right), 1.58\left(\mathrm{~s}, 8 \mathrm{H},-\mathrm{C}_{2} \mathrm{CH}_{2} \mathrm{CH}_{2} \mathrm{OH}\right), 1.42\left(\mathrm{~s}, 8 \mathrm{H},-\mathrm{C}_{2} \underline{\mathrm{C}}_{2} \mathrm{CH}_{2} \mathrm{CH}_{2} \mathrm{CH}_{2} \mathrm{OH}\right), 1.07$ (s, 4H, $-\mathrm{SiC}_{2}-$ ).

b) $\operatorname{CPOSS}(\mathrm{OH})_{2}$ and ${ }^{*} \operatorname{CPOSS}(\mathrm{OH})_{2}$. $\operatorname{VPOSS}(\mathrm{OH})_{2}(1.36 \mathrm{~g}, 1.66 \mathrm{mmol})$ or ${ }^{*} \operatorname{VPOSS}(\mathrm{OH})_{2}(1.50 \mathrm{~g}, 1.66$ mmol), 1-butanethiol (1.37 g, $14.9 \mathrm{mmol})$, and DMPA (41.0 mg, $0.16 \mathrm{mmol}$ ) were dissolved in $15 \mathrm{~mL}$ of THF. After UV irradiation for $30 \mathrm{~min}$, solvent was evaporated under vacuum. The residue was purified by silica gel chromatography with PE/EA (4/1) as eluent to afford the products as colorless oil liquid.

p-CPOSS(OH)2 $\left(1.68 \mathrm{~g}\right.$, yield: 74.6\%). ${ }^{1} \mathrm{H}$ NMR (500 MHz, $\left.\mathrm{CDCl}_{3}, \mathrm{ppm}, \delta\right): 3.76$ (t, $J=5.8 \mathrm{~Hz}, 4 \mathrm{H}$, $\mathrm{HOC}_{2} \mathrm{CH}_{2}$ ) $, 2.50-2.67\left(\mathrm{~m}, 32 \mathrm{H},-\underline{\mathrm{CH}}_{2} \mathrm{SC}_{2}{ }^{-}\right), 1.86\left(\mathrm{~h}, J=7.0 \mathrm{~Hz}, 4 \mathrm{H},-\mathrm{SCH}_{2} \mathrm{C}_{2} \mathrm{CH}_{2} \mathrm{OH}\right), 1.56(\mathrm{~m}, 12 \mathrm{H}$, $\left.-\mathrm{SCH}_{2} \mathrm{CH}_{2} \mathrm{CH}_{2} \mathrm{CH}_{3}\right), 1.43\left(\mathrm{~h}, J=7.3 \mathrm{~Hz}, 12 \mathrm{H},-\mathrm{SCH}_{2} \mathrm{CH}_{2} \mathrm{CH}_{2} \mathrm{CH}_{3}\right), 1.05$ (m, 16H, -SiC $\left.\underline{H}_{2}-\right), 0.94(18 \mathrm{H}$, $\left.-\mathrm{CH}_{2} \mathrm{CH}_{2} \mathrm{C}_{3}\right)$.

$\boldsymbol{m}$-CPOSS(OH)2 $\left(1.79\right.$ g, yield: 79.5\%). $\left.{ }^{1} \mathrm{H} \mathrm{NMR} \mathrm{(500} \mathrm{MHz,} \mathrm{CDCl}_{3}, \mathrm{ppm}, \delta\right): 3.76(\mathrm{t}, J=5.8 \mathrm{~Hz}, 4 \mathrm{H}$, $\left.\mathrm{HOC}_{2} \mathrm{CH}_{2}-\right), 2.50-2.64\left(\mathrm{~m}, 32 \mathrm{H},-\underline{\mathrm{CH}}_{2} \mathrm{SC}_{2}{ }_{2}\right), 1.85$ (h, $\left.J=7.0 \mathrm{~Hz}, 4 \mathrm{H},-\mathrm{SCH}_{2} \underline{\mathrm{C}}_{2} \mathrm{CH}_{2} \mathrm{OH}\right), 1.57(\mathrm{~m}, 12 \mathrm{H}$, $-\mathrm{SCH}_{2} \mathrm{CH}_{2} \mathrm{CH}_{2} \mathrm{CH}_{3}$ ), $1.43\left(\mathrm{~h}, J=7.3 \mathrm{~Hz}, 12 \mathrm{H},-\mathrm{SCH}_{2} \mathrm{CH}_{2} \mathrm{CH}_{2} \mathrm{CH}_{3}\right), 1.02\left(\mathrm{~m}, 16 \mathrm{H},-\mathrm{SiC}_{2}-\right), 0.93(\mathrm{~m}, 18 \mathrm{H}$, $\left.-\mathrm{CH}_{2} \mathrm{CH}_{2} \mathrm{CH}_{3}\right)$.

o-CPOSS(OH) 2 (1.93 g, yield: 85.8\%). ${ }^{1} \mathrm{H}$ NMR (500 MHz, $\left.\mathrm{CDCl}_{3}, \mathrm{ppm}, \delta\right): 3.76$ (t, $J=5.8 \mathrm{~Hz}, 4 \mathrm{H}$, $\left.\mathrm{HOC}_{2} \mathrm{CH}_{2}-\right), 2.51-2.68\left(\mathrm{~m}, 32 \mathrm{H},-\underline{\mathrm{CH}}_{2} \mathrm{SC}_{2} 2^{-}\right), 1.86\left(\mathrm{~h}, J=7.0 \mathrm{~Hz}, 4 \mathrm{H},-\mathrm{SCH}_{2} \underline{\mathrm{C}}_{2} \mathrm{CH}_{2} \mathrm{OH}\right), 1.61(\mathrm{~m}, 12 \mathrm{H}$, $-\mathrm{SCH}_{2} \underline{\mathrm{C}}_{2} \mathrm{CH}_{2} \mathrm{CH}_{3}$ ), 1.43 (h, $J=7.3 \mathrm{~Hz}, 12 \mathrm{H},-\mathrm{SCH}_{2} \mathrm{CH}_{2} \mathrm{C}_{2} \mathrm{CH}_{3}$ ), 1.03 (m, 16H, -SiC些-), 0.95 (m, 18H, $\left.-\mathrm{CH}_{2} \mathrm{CH}_{2} \mathrm{CH}_{3}\right)$.

p-*CPOSS(OH) 2 (1.55 g, yield: 68.5\%). ${ }^{1} \mathrm{H}$ NMR (500 MHz, $\left.\mathrm{CDCl}_{3}, \mathrm{ppm}, \delta\right): 3.63(\mathrm{t}, J=6.5 \mathrm{~Hz}, 4 \mathrm{H}$, $\left.\mathrm{HOC}_{2} \mathrm{CH}_{2}-\right), 2.50-2.61\left(\mathrm{~m}, 32 \mathrm{H},-\underline{\mathrm{C}}_{2} \mathrm{SC}_{2}-\right), 1.56\left(\mathrm{~m}, 20 \mathrm{H},-\mathrm{SCH}_{2} \underline{\mathrm{C}}_{2} \mathrm{CH}_{2} \mathrm{CH}_{3},-\mathrm{C}_{2} \mathrm{CH}_{2} \mathrm{CH}_{2} \mathrm{OH}\right), 1.43$

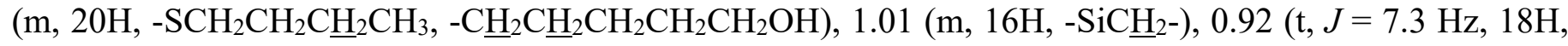
$\left.-\mathrm{CH}_{2} \mathrm{CH}_{2} \mathrm{C}_{3}\right)$.

$\boldsymbol{m}-{ }^{*} \mathbf{C P O S S}(\mathrm{OH})_{2}(1.54 \mathrm{~g}$, yield: $68.4 \%) .{ }^{1} \mathrm{H} \mathrm{NMR}\left(500 \mathrm{MHz}, \mathrm{CDCl}_{3}, \mathrm{ppm}, \delta\right): 3.65$ (t, $J=6.6 \mathrm{~Hz}, 4 \mathrm{H}$, $\left.\mathrm{HOC}_{2} \mathrm{CH}_{2}-\right), 2.53-2.61\left(\mathrm{~m}, 32 \mathrm{H},-\underline{\mathrm{CH}}_{2} \mathrm{SC}_{2}-\right), 1.54\left(\mathrm{~m}, 20 \mathrm{H},-\mathrm{SCH}_{2} \underline{\mathrm{C}}_{2} \mathrm{CH}_{2} \mathrm{CH}_{3},-\underline{\mathrm{C}}_{2} \mathrm{C}_{2} \mathrm{CH}_{2} \mathrm{OH}\right), 1.43$

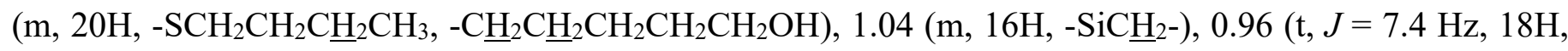
$\left.-\mathrm{CH}_{2} \mathrm{CH}_{2} \mathrm{CH}_{3}\right)$.

$\boldsymbol{o}$-*CPOSS(OH)2 $(1.60 \mathrm{~g}$, yield: $71.0 \%) .{ }^{1} \mathrm{H}$ NMR (500 MHz, $\left.\mathrm{CDCl}_{3}, \mathrm{ppm}, \delta\right): 3.61$ (t, $J=6.4 \mathrm{~Hz}, 4 \mathrm{H}$, $\left.\mathrm{HOC}_{2} \mathrm{CH}_{2}-\right), 2.51-2.64\left(\mathrm{~m}, 32 \mathrm{H},-\underline{\mathrm{C}}_{2} \mathrm{SC}_{2}-\right), 1.53\left(\mathrm{~m}, 20 \mathrm{H},-\mathrm{SCH}_{2} \underline{\mathrm{C}}_{2} \mathrm{CH}_{2} \mathrm{CH}_{3},-\mathrm{C}_{2} \mathrm{C}_{2} \mathrm{CH}_{2} \mathrm{OH}\right), 1.43$

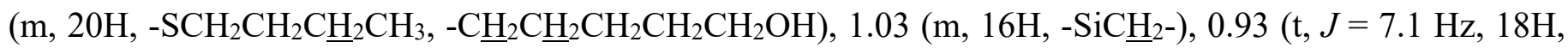
$\left.-\mathrm{CH}_{2} \mathrm{CH}_{2} \mathrm{CH}_{3}\right)$. 
c) Maleimide-protected-CPOSS-OH and Maleimide-protected-*CPOSS-OH. CPOSS $(\mathrm{OH})_{2}(2.72$ g, 2.0 mmol $)$ or ${ }^{*} \mathrm{CPOSS}(\mathrm{OH})_{2}(2.89 \mathrm{~g}, 2.0 \mathrm{mmol})$, furan protected maleimide $(0.33 \mathrm{~g}, 2.0 \mathrm{mmol})$, and triphenylphosphine $(0.79 \mathrm{~g}, 3.0 \mathrm{mmol})$ were dissolved in dry THF $(25 \mathrm{~mL})$ in a $50 \mathrm{~mL}$ round-bottom flask under argon nitrogen. Diisopropyl azodicarboxylate (DIAD, $0.61 \mathrm{~g}, 3.0 \mathrm{mmol}$ ) was then added dropwise and stirred $8 \mathrm{~h}$ under ice bath. The solvent was removed in vacuo and the crude product was purified by silica gel column chromatography with PE/EA (3/1, v/v) as eluent to afford products.

p-maleimide-protected-CPOSS-OH (1.76 g, yield: 58.6\%). ${ }^{1} \mathrm{H}$ NMR (500 MHz, $\left.\mathrm{CDCl}_{3}, \mathrm{ppm}, \delta\right): 6.52(\mathrm{~d}$, $J=1.0 \mathrm{~Hz}, 2 \mathrm{H},-\mathrm{C} \underline{\mathrm{H}}=\mathrm{C} \underline{\mathrm{H}}-), 5.26$ (t, $J=1.1 \mathrm{~Hz}, 2 \mathrm{H},-\mathrm{C} \underline{\mathrm{H}}(-\mathrm{O}-) \mathrm{C}-), 3.75$ (t, $\left.J=6.0 \mathrm{~Hz}, 2 \mathrm{H}, \mathrm{HOC}_{2} \mathrm{CH}_{2}-\right)$, $3.58\left(\mathrm{t}, J=7.1 \mathrm{~Hz}, 2 \mathrm{H},-\mathrm{NC}_{2} \mathrm{CH}_{2}-\right), 2.85(\mathrm{~s}, 2 \mathrm{H},-\mathrm{C} \underline{\mathrm{H}}(-\mathrm{C}=\mathrm{O}) \mathrm{C} \underline{\mathrm{H}}-), 2.52-2.65\left(\mathrm{~m}, 32 \mathrm{H},-\underline{\mathrm{C}}_{2} \mathrm{SC}_{2}{ }^{-}\right), 1.84$ (m, $\left.4 \mathrm{H},-\mathrm{CH}_{2} \mathrm{CH}_{2} \mathrm{CH}_{2}-\right), 1.57\left(\mathrm{~m}, 12 \mathrm{H},-\mathrm{C}_{2} \mathrm{CH}_{2} \mathrm{CH}_{3}\right), 1.42$ (dq, $\left.J=14.6,7.3 \mathrm{~Hz}, 12 \mathrm{H},-\mathrm{CH}_{2} \underline{\mathrm{C}}_{2} \mathrm{CH}_{3}\right), 1.02$ (m, $\left.16 \mathrm{H},-\mathrm{SiC}_{2} \mathrm{CH}_{2}-\right), 0.93\left(\mathrm{~m}, 18 \mathrm{H},-\mathrm{CH}_{2} \mathrm{CH}_{2} \mathrm{C}_{3}\right)$.

m-maleimide-protected-CPOSS-OH (1.55 g, yield: 51.6\%). ${ }^{1} \mathrm{H}$ NMR (500 MHz, $\mathrm{CDCl}_{3}, \mathrm{ppm}, \delta$ ): $6.52(\mathrm{~d}$, $J=0.9 \mathrm{~Hz}, 2 \mathrm{H},-\mathrm{C} \underline{\mathrm{H}}=\mathrm{C} \underline{\mathrm{H}}-), 5.24$ (t, $J=1.0 \mathrm{~Hz}, 2 \mathrm{H},-\mathrm{C} \underline{\mathrm{H}}(-\mathrm{O}-) \mathrm{C}-), 3.78$ (t, $\left.J=6.1 \mathrm{~Hz}, 2 \mathrm{H}, \mathrm{HOCH}_{2} \mathrm{CH}_{2}-\right)$, $3.52\left(\mathrm{t}, J=7.1 \mathrm{~Hz}, 2 \mathrm{H},-\mathrm{NC}_{2} \mathrm{CH}_{2}-\right), 2.84(\mathrm{~s}, 2 \mathrm{H},-\mathrm{C} \underline{\mathrm{H}}(-\mathrm{C}=\mathrm{O}) \mathrm{C} \underline{\mathrm{H}}-), 2.52-2.63\left(\mathrm{~m}, 32 \mathrm{H},-\underline{\mathrm{C}}_{2} \mathrm{SC}_{2}{ }^{-}\right), 1.83$ (m, $\left.4 \mathrm{H},-\mathrm{CH}_{2} \underline{\mathrm{C}}_{2} \mathrm{CH}_{2}-\right), 1.58\left(\mathrm{~m}, 12 \mathrm{H},-\underline{\mathrm{C}}_{2} \mathrm{CH}_{2} \mathrm{CH}_{3}\right), 1.41$ (dq, $\left.J=14.6,7.3 \mathrm{~Hz}, 12 \mathrm{H},-\mathrm{CH}_{2} \mathrm{CH}_{2} \mathrm{CH}_{3}\right), 1.03$ (m, 16H, - $\left.\mathrm{SiC}_{2} \mathrm{CH}_{2}-\right), 0.98\left(18 \mathrm{H},-\mathrm{CH}_{2} \mathrm{CH}_{2} \mathrm{C}_{3}\right)$.

o-maleimide-protected-CPOSS-OH (1.40 g, yield: 46.5\%). ${ }^{1} \mathrm{H}$ NMR (500 MHz, $\left.\mathrm{CDCl}_{3}, \mathrm{ppm}, \delta\right): 6.51(\mathrm{~d}$, $J=1.0 \mathrm{~Hz}, 2 \mathrm{H},-\mathrm{C} \underline{\mathrm{H}}=\mathrm{C} \underline{\mathrm{H}}-), 5.23$ (t, $J=1.1 \mathrm{~Hz}, 2 \mathrm{H},-\mathrm{C} \underline{\mathrm{H}}(-\mathrm{O}-) \mathrm{C}-), 3.75$ (t, $\left.J=6.8 \mathrm{~Hz}, 2 \mathrm{H}, \mathrm{HOC}_{2} \mathrm{CH}_{2}-\right)$, $3.56\left(\mathrm{t}, J=7.1 \mathrm{~Hz}, 2 \mathrm{H},-\mathrm{NC}_{2} \mathrm{CH}_{2}-\right), 2.85(\mathrm{~s}, 2 \mathrm{H},-\mathrm{C} \underline{\mathrm{H}}(-\mathrm{C}=\mathrm{O}) \mathrm{C} \underline{\mathrm{H}}-), 2.49-2.65\left(\mathrm{~m}, 32 \mathrm{H},-\underline{\mathrm{C}}_{2} \mathrm{SC}_{2}{ }^{-}\right), 1.84$ (m, $\left.4 \mathrm{H},-\mathrm{CH}_{2} \mathrm{C}_{2} \mathrm{CH}_{2}-\right), 1.56\left(\mathrm{~m}, 12 \mathrm{H},-\mathrm{CH}_{2} \mathrm{CH}_{2} \mathrm{CH}_{3}\right), 1.40$ (dq, $\left.J=14.6,7.3 \mathrm{~Hz}, 12 \mathrm{H},-\mathrm{CH}_{2} \underline{\mathrm{CH}}_{2} \mathrm{CH}_{3},\right), 1.03$ (m, $\left.16 \mathrm{H},-\mathrm{SiC}_{2} \mathrm{CH}_{2}-\right), 0.94\left(\mathrm{~m}, 18 \mathrm{H},-\mathrm{CH}_{2} \mathrm{CH}_{2} \mathrm{C}_{3}\right)$.

p-maleimide-protected-*CPOSS-OH (1.65 g, yield: 56.6\%). ${ }^{1} \mathrm{H}$ NMR (500 MHz, $\mathrm{CDCl}_{3}, \mathrm{ppm}, \delta$ ): 6.51 (d, $J=1.0 \mathrm{~Hz}, 2 \mathrm{H},-\mathrm{C} \underline{\mathrm{H}}=\mathrm{C} \underline{\mathrm{H}}-), 5.24(\mathrm{t}, J=1.0 \mathrm{~Hz}, 2 \mathrm{H},-\mathrm{C} \underline{\mathrm{H}}(-\mathrm{O}-) \mathrm{C}-), 3.62$ (t, $\left.J=6.5 \mathrm{~Hz}, 2 \mathrm{H}, \mathrm{HOC}_{2} \mathrm{CH}_{2}-\right)$, $3.46\left(\mathrm{t}, J=7.6 \mathrm{~Hz}, 2 \mathrm{H},-\mathrm{NC}_{2} \mathrm{CH}_{2}-\right), 2.87(\mathrm{~s}, 2 \mathrm{H},-\mathrm{C} \underline{\mathrm{H}}(-\mathrm{C}=\mathrm{O}) \mathrm{C} \underline{\mathrm{H}}-), 2.52-2.61\left(\mathrm{~m}, 32 \mathrm{H},-\underline{\mathrm{C}}_{2} \mathrm{SC}_{2}-\right), 1.54$ (pd, $\left.J=7.4 \mathrm{~Hz}, 5.4 \mathrm{~Hz}, 20 \mathrm{H},-\mathrm{C}_{2} \mathrm{CH}_{2} \mathrm{CH}_{3},-\mathrm{SCH}_{2} \mathrm{CH}_{2} \mathrm{CH}_{2} \underline{\mathrm{C}}_{2} \mathrm{C}_{2} \mathrm{CH}_{2}-\right), 1.40$ (h, $J=7.2 \mathrm{~Hz}, 20 \mathrm{H}$, $\left.-\mathrm{CH}_{2} \underline{\mathrm{C}}_{2} \mathrm{CH}_{3},-\mathrm{SCH}_{2} \underline{\mathrm{C}}_{2} \mathrm{C}_{2} \mathrm{CH}_{2} \mathrm{CH}_{2} \mathrm{CH}_{2}-\right), 1.01$ (m, 16H, $\left.-\mathrm{SiC}_{2} \mathrm{CH}_{2}-\right), 0.93$ (m, J $7.1 \mathrm{~Hz} 18 \mathrm{H}$, $\left.-\mathrm{CH}_{2} \mathrm{CH}_{2} \mathrm{C}_{3}\right)$.

m-maleimide-protected-*CPOSS-OH (1.61 g, yield: 56.1\%). ${ }^{1} \mathrm{H}$ NMR (500 MHz, CDCl 3 , ppm, $\delta$ ): 6.53 (d, $J=1.0 \mathrm{~Hz}, 2 \mathrm{H},-\mathrm{C} \underline{\mathrm{H}}=\mathrm{C} \underline{\mathrm{H}}-), 5.25$ (t, $J=1.0 \mathrm{~Hz}, 2 \mathrm{H},-\mathrm{C} \underline{\mathrm{H}}(-\mathrm{O}-) \mathrm{C}-), 3.63$ (t, $\left.J=6.6 \mathrm{~Hz}, 2 \mathrm{H}, \mathrm{HOCH}_{2} \mathrm{CH}_{2}-\right)$, $3.46\left(\mathrm{t}, J=7.4 \mathrm{~Hz}, 2 \mathrm{H},-\mathrm{NC}_{2} \mathrm{CH}_{2}-\right), 2.85$ (s, 2H, - $\left.\underline{\mathrm{H}}(-\mathrm{C}=\mathrm{O}) \mathrm{C} \underline{\mathrm{H}}-\right), 2.48-2.61$ (m, 32H, - $\left.\underline{\mathrm{CH}}_{2} \mathrm{SC}_{2}{ }^{-}\right), 1.53$ (pd, $J=7.4 \mathrm{~Hz}, 5.4 \mathrm{~Hz}, 20 \mathrm{H},-\mathrm{C}_{2} \mathrm{CH}_{2} \mathrm{CH}_{3},-\mathrm{SCH}_{2} \mathrm{CH}_{2} \mathrm{CH}_{2} \underline{\mathrm{C}}_{2} \underline{\mathrm{C}}_{2} \mathrm{CH}_{2}-$ ), 1.40 (h, $J=7.2 \mathrm{~Hz}, 20 \mathrm{H}$, 
$\left.-\mathrm{CH}_{2} \mathrm{CH}_{2} \mathrm{CH}_{3},-\mathrm{SCH}_{2} \underline{\mathrm{CH}}_{2} \mathrm{CH}_{2} \mathrm{CH}_{2} \mathrm{CH}_{2} \mathrm{CH}_{2}-\right), 1.01$ (m, 16H, $\left.-\mathrm{SiCH}_{2} \mathrm{CH}_{2}-\right), 0.92$ (m, J = $7.4 \mathrm{~Hz} 18 \mathrm{H}$, $\left.-\mathrm{CH}_{2} \mathrm{CH}_{2} \mathrm{CH}_{3}\right)$.

o-maleimide-protected-*CPOSS-OH (1.51 g, yield: 54.9\%). ${ }^{1} \mathrm{H}$ NMR (500 MHz, $\left.\mathrm{CDCl}_{3}, \mathrm{ppm}, \delta\right): 6.50(\mathrm{~d}$, $J=1.0 \mathrm{~Hz}, 2 \mathrm{H},-\mathrm{C} \underline{\mathrm{H}}=\mathrm{C} \underline{\mathrm{H}}-), 5.25$ (t, $J=1.0 \mathrm{~Hz}, 2 \mathrm{H},-\mathrm{C} \underline{\mathrm{H}}(-\mathrm{O}-) \mathrm{C}-), 3.63$ (t, $\left.J=6.6 \mathrm{~Hz}, 2 \mathrm{H}, \mathrm{HOC}_{2} \mathrm{CH}_{2}-\right)$, $3.46\left(\mathrm{t}, J=7.4 \mathrm{~Hz}, 2 \mathrm{H},-\mathrm{NC}_{2} \mathrm{CH}_{2}-\right), 2.85(\mathrm{~s}, 2 \mathrm{H},-\mathrm{C} \underline{\mathrm{H}}(-\mathrm{C}=\mathrm{O}) \mathrm{C} \underline{\mathrm{H}}-), 2.50-2.61\left(\mathrm{~m}, 32 \mathrm{H},-\underline{\mathrm{C}}_{2} \mathrm{SC}_{2}-\right), 1.57$ (pd, $\left.J=7.4 \mathrm{~Hz}, 5.4 \mathrm{~Hz}, 20 \mathrm{H},-\mathrm{CH}_{2} \mathrm{CH}_{2} \mathrm{CH}_{3},-\mathrm{SCH}_{2} \mathrm{CH}_{2} \mathrm{CH}_{2} \underline{\mathrm{C}}_{2} \mathrm{CH}_{2} \mathrm{CH}_{2}-\right), 1.41$ (h, $J=7.2 \mathrm{~Hz}, 20 \mathrm{H}$, $\left.-\mathrm{CH}_{2} \mathrm{CH}_{2} \mathrm{CH}_{3},-\mathrm{SCH}_{2} \mathrm{CH}_{2} \mathrm{CH}_{2} \mathrm{CH}_{2} \mathrm{CH}_{2} \mathrm{CH}_{2}-\right), 1.03$ (m, 16H, - $\left.\mathrm{SiCH}_{2} \mathrm{CH}_{2}-\right), 0.92$ (m, $J=7.3 \mathrm{~Hz} 18 \mathrm{H}$, $\left.-\mathrm{CH}_{2} \mathrm{CH}_{2} \underline{\mathrm{CH}}_{3}\right)$.

d) CPOSS monomer and *CPOSS monomer. Maleimide-protected-CPOSS-OH (1.51 g, $1.0 \mathrm{mmol})$ or maleimide-protected-*CPOSS-OH (1.60 g, $1.0 \mathrm{mmol})$ and $\mathrm{CBr}_{4}(0.66 \mathrm{~g}, 2.0 \mathrm{mmol})$ were dissolved in $15 \mathrm{~mL}$ of anhydrous $\mathrm{CH}_{2} \mathrm{Cl}_{2}$ in a $25 \mathrm{~mL}$ round-bottom flask. $\mathrm{PPh}_{3}(0.52 \mathrm{~g}, 2.0 \mathrm{mmol})$ was then slowly added and string for 4 hours. After that, $20 \mathrm{~mL}$ of $\mathrm{MeOH}, \mathrm{KI}(20 \mathrm{mg})$, and potassium thioacetate $(0.23 \mathrm{~g}, 2.0 \mathrm{mmol})$ were added, and the mixture was further stirred at $75{ }^{\circ} \mathrm{C}$ for $12 \mathrm{~h}$ under argon atmosphere. After removal of solvent, the crude material was purified by automated column chromatography using PE/EA (5/1, v/v) as eluent. The pure monomer was obtained as colorless oil.

p-CPOSS-monomer (1.32 g, yield: 84.3\%). ${ }^{1} \mathrm{H}$ NMR (500 MHz, $\left.\mathrm{CDCl}_{3}, \mathrm{ppm}, \delta\right): 6.51(\mathrm{~d}, J=1.0 \mathrm{~Hz}, 2 \mathrm{H}$, $-\mathrm{C} \underline{\mathrm{H}}=\mathrm{C} \underline{\mathrm{H}}-), 5.26$ (t, $J=0.8 \mathrm{~Hz}, 2 \mathrm{H},-\mathrm{C} \underline{\mathrm{H}}(-\mathrm{O}-) \mathrm{C}-), 3.58$ (t, $\left.J=7.1 \mathrm{~Hz}, 2 \mathrm{H},-\mathrm{NC}_{2} \mathrm{CH}_{2}-\right), 2.96$ (t, $J=7.2 \mathrm{~Hz}$, $\left.2 \mathrm{H},-(\mathrm{O}=\mathrm{C}) \mathrm{SC}_{2} \mathrm{CH}_{2}-\right), 2.85$ (s, 2H, - $\left.\mathrm{CH}(-\mathrm{C}=\mathrm{O}) \mathrm{CH}-\right), 2.48-2.61\left(\mathrm{~m}, 32 \mathrm{H},-\mathrm{C}_{2} \mathrm{SC}_{2}{ }_{2}\right), 2.33$ (t, $J=7.4 \mathrm{~Hz}$, $\left.3 \mathrm{H}, \underline{\mathrm{C}}_{3}(\mathrm{O}=\mathrm{C}-) \mathrm{SCH}_{2} \mathrm{CH}_{2}-\right), 1.84\left(\mathrm{td}, J=7.2,5.4 \mathrm{~Hz}, 4 \mathrm{H},-\mathrm{CH}_{2} \underline{\mathrm{C}}_{2} \mathrm{CH}_{2} \mathrm{~S}-\right), 1.55$ (pd, $J=7.9,7.5,2.5 \mathrm{~Hz}$, $12 \mathrm{H},-\underline{\mathrm{H}}_{2} \mathrm{CH}_{2} \mathrm{CH}_{3}$ ), 1.40 (hd, $\left.J=7.3,1.6 \mathrm{~Hz}, 12 \mathrm{H},-\mathrm{CH}_{2} \underline{\mathrm{C}}_{2} \mathrm{CH}_{3}\right), 1.01$ (m, 16H, $\left.-\mathrm{SiC}_{2} \mathrm{CH}_{2}-\right), 0.92$ (m, $\left.18 \mathrm{H},-\mathrm{CH}_{2} \mathrm{CH}_{2} \mathrm{CH}_{3}\right)$.

m-CPOSS-monomer (1.27 g, yield: 80.9\%). ${ }^{1} \mathrm{H}$ NMR (500 MHz, $\left.\mathrm{CDCl}_{3}, \mathrm{ppm}, \delta\right): 6.50$ (d, J=1.0 Hz, 2H, $-\mathrm{C} \underline{\mathrm{H}}=\mathrm{C} \underline{\mathrm{H}}-), 5.27$ (t, $J=0.8 \mathrm{~Hz}, 2 \mathrm{H},-\mathrm{C} \underline{\mathrm{H}}(-\mathrm{O}-) \mathrm{C}-), 3.56$ (t, $\left.J=7.1 \mathrm{~Hz}, 2 \mathrm{H},-\mathrm{NCH}_{2} \mathrm{CH}_{2}-\right), 2.96$ (t, $J=7.2 \mathrm{~Hz}$, $\left.2 \mathrm{H},-(\mathrm{O}=\mathrm{C}) \mathrm{SC}_{2} \mathrm{CH}_{2}-\right), 2.84(\mathrm{~s}, 2 \mathrm{H},-\mathrm{C} \underline{\mathrm{H}}(-\mathrm{C}=\mathrm{O}) \mathrm{C} \underline{\mathrm{H}}-), 2.48-2.61\left(\mathrm{~m}, 32 \mathrm{H},-\underline{\mathrm{C}}_{2} \mathrm{SC}_{\mathrm{H}_{2}}\right), 2.38$ (t, $J=7.3 \mathrm{~Hz}$, $\left.3 \mathrm{H}, \mathrm{C}_{3}(\mathrm{O}=\mathrm{C}-) \mathrm{SCH}_{2} \mathrm{CH}_{2}-\right), 1.85\left(\mathrm{td}, J=7.2,5.5 \mathrm{~Hz}, 4 \mathrm{H},-\mathrm{CH}_{2} \underline{\mathrm{C}}_{2} \mathrm{CH}_{2} \mathrm{~S}-\right), 1.55$ (pd, $J=7.9,7.5,2.6 \mathrm{~Hz}$, $12 \mathrm{H},-\underline{\mathrm{C}}_{2} \mathrm{CH}_{2} \mathrm{CH}_{3}$ ), 1.41 (hd, $\left.J=72,1.6 \mathrm{~Hz}, 12 \mathrm{H},-\mathrm{CH}_{2} \mathrm{C}_{2} \mathrm{CH}_{3}\right), 1.04$ (m, 16H, $\left.-\mathrm{SiC}_{2} \mathrm{CH}_{2}-\right), 0.94(\mathrm{~m}$, $\left.18 \mathrm{H},-\mathrm{CH}_{2} \mathrm{CH}_{2} \mathrm{CH}_{3}\right)$.

o-CPOSS-monomer (1.29 g, yield: 82.5\%). ${ }^{1} \mathrm{H}$ NMR (500 MHz, $\left.\mathrm{CDCl}_{3}, \mathrm{ppm}, \delta\right): 6.51(\mathrm{~d}, J=1.0 \mathrm{~Hz}, 2 \mathrm{H}$, $-\mathrm{C} \underline{\mathrm{H}}=\mathrm{C} \underline{\mathrm{H}}-), 5.26$ (t, $J=0.8 \mathrm{~Hz}, 2 \mathrm{H},-\mathrm{C} \underline{\mathrm{H}}(-\mathrm{O}-) \mathrm{C}-), 3.58$ (t, $J=7.3 \mathrm{~Hz}, 2 \mathrm{H},-\mathrm{NC}_{2} \mathrm{CH}_{2}$ ), 2.94 (t, $J=7.4 \mathrm{~Hz}$, $\left.2 \mathrm{H},-(\mathrm{O}=\mathrm{C}) \mathrm{SC} \underline{H}_{2} \mathrm{CH}_{2}-\right), 2.85(\mathrm{~s}, 2 \mathrm{H},-\mathrm{CH}(-\mathrm{C}=\mathrm{O}) \mathrm{CH}-), 2.48-2.63\left(\mathrm{~m}, 32 \mathrm{H},-\underline{\mathrm{C}}_{2} \mathrm{SC}_{2}{ }_{2}\right), 2.36(\mathrm{t}, J=7.2 \mathrm{~Hz}$, $\left.3 \mathrm{H}, \mathrm{CH}_{3}(\mathrm{O}=\mathrm{C}-) \mathrm{SCH}_{2} \mathrm{CH}_{2}-\right), 1.82\left(\mathrm{td}, J=7.1,5.4 \mathrm{~Hz}, 4 \mathrm{H},-\mathrm{CH}_{2} \underline{\mathrm{CH}}_{2} \mathrm{CH}_{2} \mathrm{~S}-\right), 1.57$ (pd, $J=7.9,7.6,2.5 \mathrm{~Hz}$, 
$12 \mathrm{H},-\mathrm{CH}_{2} \mathrm{CH}_{2} \mathrm{CH}_{3}$ ), 1.42 (hd, $\left.J=7.3,1.77 \mathrm{~Hz}, 12 \mathrm{H},-\mathrm{CH}_{2} \mathrm{CH}_{2} \mathrm{CH}_{3}\right), 1.04$ (m, 16H, $\left.-\mathrm{SiC}_{2} \mathrm{CH}_{2}-\right), 0.92$ (m, $\left.18 \mathrm{H},-\mathrm{CH}_{2} \mathrm{CH}_{2} \mathrm{CH}_{3}\right)$.

p-*CPOSS-monomer (1.15 g, yield: 74.3\%). ${ }^{1} \mathrm{H}$ NMR (500 MHz, $\left.\mathrm{CDCl}_{3}, \mathrm{ppm}, \delta\right): 6.48(\mathrm{~d}, J=1.0 \mathrm{~Hz}, 2 \mathrm{H}$, $-\mathrm{C} \underline{\mathrm{H}}=\mathrm{C} \underline{\mathrm{H}}-), 5.22$ (t, $J=0.8 \mathrm{~Hz}, 2 \mathrm{H},-\mathrm{C} \underline{\mathrm{H}}(-\mathrm{O}-) \mathrm{C}-), 3.43$ (t, $\left.J=7.3 \mathrm{~Hz}, 2 \mathrm{H},-\mathrm{NC}_{2} \mathrm{CH}_{2}-\right), 2.83$ (t, $J=7.3 \mathrm{~Hz}$, $\left.2 \mathrm{H},-(\mathrm{O}=\mathrm{C}) \mathrm{SC}_{2} \mathrm{CH}_{2}-\right), 2.80(\mathrm{~s}, 2 \mathrm{H},-\mathrm{C} \underline{\mathrm{H}}(-\mathrm{C}=\mathrm{O}) \mathrm{C} \underline{\mathrm{H}}-), 2.43-2.62\left(\mathrm{~m}, 32 \mathrm{H},-\underline{\mathrm{C}}_{2} \mathrm{SC}_{2_{2}}\right), 2.29(\mathrm{~s}, 3 \mathrm{H}$, $\left.\mathrm{C}_{3}(\mathrm{O}=\mathrm{C}-) \mathrm{SCH}_{2} \mathrm{CH}_{2}-\right), \quad 1.52\left(\mathrm{~m}, 20 \mathrm{H},-\mathrm{C}_{2} \mathrm{CH}_{2} \mathrm{CH}_{3},-\mathrm{SCH}_{2} \mathrm{CH}_{2} \mathrm{CH}_{2} \mathrm{C}_{2} \mathrm{C}_{2} \mathrm{CH}_{2}-\right), \quad 1.39 \quad(\mathrm{~m}, 20 \mathrm{H}$, $-\mathrm{CH}_{2} \underline{\mathrm{C}}_{2} \mathrm{CH}_{3},-\mathrm{SCH}_{2} \underline{\mathrm{CH}}_{2} \mathrm{C}_{2} \mathrm{CH}_{2} \mathrm{CH}_{2} \mathrm{CH}_{2}$ ), 0.98 (ddd, $\left.J=12.9,7.2 \mathrm{~Hz}, 16 \mathrm{H},-\mathrm{SiC}_{2} \mathrm{CH}_{2}-\right), 0.89$ (t, $18 \mathrm{H}, J$ $\left.=7.3 \mathrm{~Hz},-\mathrm{CH}_{2} \mathrm{CH}_{2} \underline{\mathrm{CH}}_{3}\right)$.

m- *CPOSS-monomer (1.22 g, yield: 77.3\%). ${ }^{1} \mathrm{H}$ NMR (500 MHz, $\left.\mathrm{CDCl}_{3}, \mathrm{ppm}, \delta\right): 6.46(\mathrm{~d}, J=1.0 \mathrm{~Hz}, 2 \mathrm{H}$, $-\mathrm{C} \underline{\mathrm{H}}=\mathrm{C} \underline{\mathrm{H}}-), 5.23$ (t, $J=0.9 \mathrm{~Hz}, 2 \mathrm{H},-\mathrm{C} \underline{\mathrm{H}}(-\mathrm{O}-) \mathrm{C}-), 3.42$ (t, $J=7.3 \mathrm{~Hz}, 2 \mathrm{H},-\mathrm{NC}_{2} \mathrm{CH}_{2}$ ), 2.81 (t, $J=7.3 \mathrm{~Hz}$, $\left.2 \mathrm{H},-(\mathrm{O}=\mathrm{C}) \mathrm{SCH}_{2} \mathrm{CH}_{2}-\right), 2.82(\mathrm{~s}, 2 \mathrm{H},-\mathrm{C} \underline{\mathrm{H}}(-\mathrm{C}=\mathrm{O}) \mathrm{C} \underline{\mathrm{H}}-), 2.43-2.60\left(\mathrm{~m}, 32 \mathrm{H},-\mathrm{C}_{2} \mathrm{SC}_{\mathrm{H}_{2}}\right), 2.3(\mathrm{~s}, 3 \mathrm{H}$, $\left.\mathrm{C}_{3}(\mathrm{O}=\mathrm{C}-) \mathrm{SCH}_{2} \mathrm{CH}_{2}-\right), \quad 1.51$ (m, 20H, $\left.-\mathrm{CH}_{2} \mathrm{CH}_{2} \mathrm{CH}_{3},-\mathrm{SCH}_{2} \mathrm{CH}_{2} \mathrm{CH}_{2} \mathrm{CH}_{2} \mathrm{C}_{2} \mathrm{CH}_{2}-\right), 1.38 \quad(\mathrm{~m}, 20 \mathrm{H}$, $-\mathrm{CH}_{2} \underline{\mathrm{C}}_{2} \mathrm{CH}_{3},-\mathrm{SCH}_{2} \underline{\mathrm{C}}_{2} \mathrm{C}_{2} \mathrm{CH}_{2} \mathrm{CH}_{2} \mathrm{CH}_{2}$ ), 1.01 (ddd, $J=12.9,7.2 \mathrm{~Hz}, 16 \mathrm{H},-\mathrm{SiC}_{2} \mathrm{CH}_{2}$ ), 0.90 (t, $18 \mathrm{H}, J$ $\left.=7.3 \mathrm{~Hz},-\mathrm{CH}_{2} \mathrm{CH}_{2} \underline{\mathrm{CH}}_{3}\right)$.

o- *CPOSS-monomer (1.04 g, yield: $67.2 \%) .{ }^{1} \mathrm{H}$ NMR (500 MHz, $\left.\mathrm{CDCl}_{3}, \mathrm{ppm}, \delta\right): 6.48$ (d, $J=1.0 \mathrm{~Hz}, 2 \mathrm{H}$, $-\mathrm{C} \underline{\mathrm{H}}=\mathrm{C} \underline{\mathrm{H}}-), 5.20$ (t, $J=0.8 \mathrm{~Hz}, 2 \mathrm{H},-\mathrm{C} \underline{\mathrm{H}}(-\mathrm{O}-) \mathrm{C}-), 3.43$ (t, $\left.J=7.3 \mathrm{~Hz}, 2 \mathrm{H},-\mathrm{NC}_{2} \mathrm{CH}_{2}-\right), 2.83$ (t, $J=7.3 \mathrm{~Hz}$, $\left.2 \mathrm{H},-(\mathrm{O}=\mathrm{C}) \mathrm{SCH}_{2} \mathrm{CH}_{2}-\right), 2.81(\mathrm{~s}, 2 \mathrm{H},-\mathrm{CH}(-\mathrm{C}=\mathrm{O}) \mathrm{C} \underline{\mathrm{H}}-), 2.40-2.62\left(\mathrm{~m}, 32 \mathrm{H},-\mathrm{C}_{2} \mathrm{SC}_{2}-\right), 2.28(\mathrm{~s}, 3 \mathrm{H}$, $\left.\mathrm{C}_{3}(\mathrm{O}=\mathrm{C}-) \mathrm{SCH}_{2} \mathrm{CH}_{2}-\right), 1.50 \quad\left(\mathrm{~m}, 20 \mathrm{H},-\mathrm{C}_{2} \mathrm{CH}_{2} \mathrm{CH}_{3},-\mathrm{SCH}_{2} \mathrm{CH}_{2} \mathrm{CH}_{2} \mathrm{C}_{2} \mathrm{C}_{2} \mathrm{CH}_{2}-\right), 1.40 \quad(\mathrm{~m}, 20 \mathrm{H}$, $-\mathrm{CH}_{2} \underline{\mathrm{C}}_{2} \mathrm{CH}_{3},-\mathrm{SCH}_{2} \underline{\mathrm{C}}_{2} \mathrm{C}_{2} \mathrm{CH}_{2} \mathrm{CH}_{2} \mathrm{CH}_{2}$ ), 0.99 (ddd, $J=12.9,7.3 \mathrm{~Hz}, 16 \mathrm{H},-\mathrm{SiC}_{2} \mathrm{CH}_{2}$ ), 0.91 (t, $18 \mathrm{H}, J$ $\left.=7.3 \mathrm{~Hz},-\mathrm{CH}_{2} \mathrm{CH}_{2} \mathrm{CH}_{3}\right)$.

\subsection{Discrete CPOSS chains $\mathrm{C}_{n}$ and ${ }^{*} \mathrm{C}_{n}$}

The syntheses of discrete CPOSS chains $\left(\mathrm{C}_{n}\right.$ and $\left.{ }^{*} \mathrm{C}_{n}\right)$ follow similar protocol. Here we take $p$ - $\mathrm{C}_{n}$ as a representative example to illustrate the synthetic details.

\section{a) Synthesis of $p-C_{2}$}

p-C $\mathbf{C}_{\mathbf{1}}$-mal: To a $100 \mathrm{~mL}$ round-bottomed flask equipped with a condenser, monomer (1.56 g, $\left.1.0 \mathrm{mmol}\right)$ was dissolved in $30 \mathrm{~mL}$ of toluene. The mixture was stirred in an oil bath at $120{ }^{\circ} \mathrm{C}$ under argon flow for about 6 h. TLC showed the reaction was complete. After cooling to room temperature, toluene was removed by rotary evaporation. And the residue was dried under vacuum at $25^{\circ} \mathrm{C}$ for $24 \mathrm{~h}$ to afford $\boldsymbol{p}$ - $\mathbf{C}_{\mathbf{1}}$-mal as colorless oil liquid (1.47 g, yield: 98\%). 
p-C1-SH: Monomer (1.56 g, $1.0 \mathrm{mmol})$ was added to a $50 \mathrm{~mL}$ round-bottom flask and dissolved in $40 \mathrm{~mL}$ of $\mathrm{MeOH} / \mathrm{CH}_{2} \mathrm{Cl}_{2}$ 1:2 mixed solvent. The solution was cooled to $-78^{\circ} \mathrm{C}$ and string for 10 minutes. With string, the acetyl chloride $(15.0 \mathrm{~mL}, 0.11 \mathrm{~mol})$ was added dropwise to the solution. The solution was further stirred at ambient temperature for $12 \mathrm{~h}$. After reaction, the mixture was poured into $100 \mathrm{~mL}$ deionized water and extracted with DCM. The organic layer was washed with deionized water three times and dried with anhydrous $\mathrm{Na}_{2} \mathrm{SO}_{4}$. The solvent was removed by rotary evaporation and final product $\boldsymbol{p}$ - $\mathbf{C}_{\mathbf{1}}-\mathbf{S H}$ was obtained as colorless oil liquid (1.43 g, yield: 94\%).

p-C $\mathbf{2}$ : p-C $\mathbf{1}-\mathbf{m a l}(1.47 \mathrm{~g})$ and $\boldsymbol{p}$ - $\mathbf{C}_{\mathbf{1}}-\mathbf{S H}(1.43 \mathrm{~g})$ were dissolved in $25 \mathrm{~mL}$ of dry $\mathrm{CHCl}_{3}$ in a $50 \mathrm{~mL}$ two-neck round-bottom flask equipped with a $25 \mathrm{~mL}$ slow-addition apparatus under argon atmosphere at $25^{\circ} \mathrm{C}$. TEA $(1.0 \mathrm{~mL}, 7.3 \mathrm{mmol})$ was added dropwise to the solution and the mixture was stirred for about $24 \mathrm{~h}$. The reaction mixture was quenched with $20 \mathrm{~mL}$ water and washed with $30 \mathrm{~mL}$ saturated $\mathrm{NaHCO}_{3}$ (aq.). The combined organic layer was dried with anhydrous $\mathrm{Na}_{2} \mathrm{SO}_{4}$ and the solvent was evaporated to afford the crude product which was purified by column chromatography over silica gel eluting with PE/EA (4/1) to give the $\boldsymbol{p}-\mathbf{C}_{2}$ as colorless oil liquid.

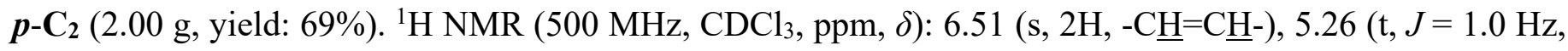

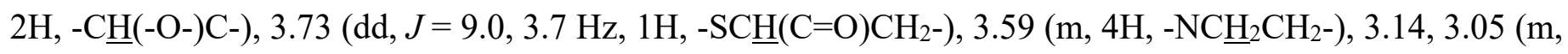
$\left.2 \mathrm{H},-\mathrm{CHCH}_{2}(\mathrm{C}=\mathrm{O})-\right), 2.97\left(\mathrm{~s}, 2 \mathrm{H}, \mathrm{CH}_{3}(\mathrm{O}=\mathrm{C}) \mathrm{SC}_{2} \mathrm{CH}_{2}-\right), 2.85$ (t, $\left.J=5.5 \mathrm{~Hz}, 2 \mathrm{H},-\mathrm{C} \underline{\mathrm{H}}(-\mathrm{C}=\mathrm{O}) \mathrm{C} \underline{\mathrm{H}}-\right)$, 2.47-2.63 (m, 66H, - $\left.\underline{\mathrm{C}}_{2} \mathrm{SC}_{\mathrm{H}_{2}}, \quad-\underline{\mathrm{C}}_{2} \mathrm{SCH}-\right), 2.34 \quad\left(\mathrm{~s}, 3 \mathrm{H}, \quad \mathrm{C}_{3}(\mathrm{O}=\mathrm{C}-) \mathrm{SCH}_{2} \mathrm{CH}_{2}-\right), 1.86 \quad(\mathrm{~m}, 8 \mathrm{H}$, $\left.-\mathrm{CH}_{2} \mathrm{CH}_{2} \mathrm{CH}_{2} \mathrm{~S}-\right), 1.56\left(\mathrm{~m}, 24 \mathrm{H},-\mathrm{CH}_{2} \mathrm{CH}_{2} \mathrm{CH}_{3}\right), 1.42\left(\mathrm{~m}, 24 \mathrm{H},-\mathrm{CH}_{2} \mathrm{CH}_{2} \mathrm{CH}_{3}\right), 1.02\left(\mathrm{~m}, 32 \mathrm{H},-\mathrm{SiCH}_{2} \mathrm{CH}_{2}-\right)$, $0.93\left(\mathrm{~m}, 36 \mathrm{H},-\mathrm{CH}_{2} \mathrm{CH}_{2} \mathrm{CH}_{3}\right)$.

\section{b) Synthesis of $p-C_{4}$}

p-C $\mathbf{C}_{\mathbf{2}}$-mal: To a $50 \mathrm{~mL}$ round-bottomed flask equipped with a condenser, $\boldsymbol{p}$ - $\mathbf{C}_{\mathbf{2}}(0.3 \mathrm{mmol}, 0.91 \mathrm{~g})$ was dissolved in $20 \mathrm{~mL}$ of toluene. The mixture was stirred in an oil bath at $120{ }^{\circ} \mathrm{C}$ under argon flow for about 6 h. TLC showed the reaction was complete. After cooling to room temperature, toluene was removed by rotary evaporation. And the residue was dried under vacuum at $25{ }^{\circ} \mathrm{C}$ for $24 \mathrm{~h}$ to afford $\boldsymbol{p}$ - $\mathbf{C}_{2}$-mal as colorless oil liquid (0.81 g, yield: 92\%).

p-C2-SH: $\boldsymbol{p}-\mathbf{C}_{2}(0.3 \mathrm{mmol}, 0.91 \mathrm{~g})$ was added to a $50 \mathrm{~mL}$ round-bottom flask and dissolved in $30 \mathrm{~mL}$ of $\mathrm{MeOH} / \mathrm{CH}_{2} \mathrm{Cl}_{2}$ 1:2 mixed solvent. The solution was cooled to $-78^{\circ} \mathrm{C}$ and string for 10 minutes. With string, the acetyl chloride $(8.0 \mathrm{~mL}, 59 \mathrm{mmol})$ was added dropwise to the solution. The solution was further stirred at ambient temperature for $12 \mathrm{~h}$. After reaction, the mixture was poured into $100 \mathrm{~mL}$ deionized water and extracted with DCM. The organic layer was washed with deionized water three times and dried with 
anhydrous $\mathrm{Na}_{2} \mathrm{SO}_{4}$. The solvent was removed by rotary evaporation and final product $\boldsymbol{p}-\mathbf{C}_{2}-\mathbf{S H}$ was obtained as colorless oil liquid ( $0.79 \mathrm{~g}$, yield: $89 \%)$.

p-C4: $\boldsymbol{p}$ - $\mathbf{C}_{2}$-mal $(0.81 \mathrm{~g})$ and $\boldsymbol{p}$ - $\mathbf{C}_{2}$-SH $(0.79 \mathrm{~g})$ were dissolved in $20 \mathrm{~mL}$ of dry $\mathrm{CHCl}_{3}$ in a $50 \mathrm{~mL}$ two-neck round-bottom flask equipped with a $25 \mathrm{~mL}$ slow-addition apparatus under argon atmosphere at $25^{\circ} \mathrm{C}$. TEA $(0.8 \mathrm{~mL}, 5.8 \mathrm{mmol})$ was added dropwise to the solution and the mixture was stirred for about $24 \mathrm{~h}$. The reaction mixture was washed with $30 \mathrm{~mL}$ saturated $\mathrm{NaHCO}_{3}$ (aq.). The combined organic layer was dried with anhydrous $\mathrm{Na}_{2} \mathrm{SO}_{4}$ and the solvent was evaporated to afford the crude product which was purified by recycling preparative $\mathrm{SEC}$ to give the $\boldsymbol{p}-\mathbf{C}_{\mathbf{4}}$ as colorless oil liquid.

p-C 4 (0.88 g, yield: 55\%). ${ }^{1} \mathrm{H}$ NMR $\left(500 \mathrm{MHz}, \mathrm{CDCl}_{3}, \mathrm{ppm}, \delta\right): 6.52$ ( t, $\left.J=1.0 \mathrm{~Hz}, 2 \mathrm{H},-\mathrm{C} \underline{\mathrm{H}}=\mathrm{C} \underline{\mathrm{H}}-\right), 5.26(\mathrm{t}$, $J=1.0 \mathrm{~Hz}, 2 \mathrm{H},-\mathrm{CH}(-\mathrm{O}-) \mathrm{C}-), 3.74\left(\mathrm{dd}, J=9.0,3.6 \mathrm{~Hz}, 3 \mathrm{H},-\mathrm{SC} \underline{\mathrm{H}}(\mathrm{C}=\mathrm{O}) \mathrm{CH}_{2-}\right), 3.58(\mathrm{dt}, J=13.6,7.2 \mathrm{~Hz}, 8 \mathrm{H}$, $\left.-\mathrm{NC}_{2} \mathrm{CH}_{2}-\right), 3.14,3.05$ (m, 6H, $\left.-\mathrm{CHCH}_{2}(\mathrm{C}=\mathrm{O})-\right), 2.97$ (t, $\left.J=7.2 \mathrm{~Hz}, 2 \mathrm{H}, \mathrm{CH}_{3}(\mathrm{O}=\mathrm{C}) \mathrm{SC}_{2} \mathrm{CH}_{2}-\right), 2.84$ (s, $2 \mathrm{H},-\mathrm{C} \underline{\mathrm{H}}(-\mathrm{C}=\mathrm{O}) \mathrm{C} \underline{\mathrm{H}}-), 2.44-2.63\left(\mathrm{~m}, 134 \mathrm{H},-\mathrm{C}_{2} \mathrm{SCH}_{2}-,-\underline{\mathrm{C}}_{2} \mathrm{SCH}-\right), 2.36\left(\mathrm{~s}, 3 \mathrm{H}, \mathrm{CH}_{3}(\mathrm{O}=\mathrm{C}-) \mathrm{SCH}_{2} \mathrm{CH}_{2}-\right)$, 1.85 (m, 16H, - $\left.\mathrm{CH}_{2} \underline{\mathrm{C}}_{2} \mathrm{CH}_{2} \mathrm{~S}-\right), 1.57$ (m, 48H, $\left.-\underline{\mathrm{C}}_{2} \mathrm{CH}_{2} \mathrm{CH}_{3}\right), 1.42\left(\mathrm{~m}, 48 \mathrm{H},-\mathrm{CH}_{2} \mathrm{C}_{2} \mathrm{CH}_{3}\right), 1.03$ (m, 64H, $\left.-\mathrm{SiCH}_{2} \mathrm{CH}_{2}-\right), 0.94\left(\mathrm{t}, J=7.9 \mathrm{~Hz}, 72 \mathrm{H},-\mathrm{CH}_{2} \mathrm{CH}_{2} \mathrm{CH}_{3}\right)$.

\section{c) Synthesis of $p-C_{8}$}

p-C 4 -mal: To a $50 \mathrm{~mL}$ round-bottomed flask equipped with a condenser, $\boldsymbol{p}$ - $\mathbf{C}_{\mathbf{4}}(0.07 \mathrm{mmol}, 0.41 \mathrm{~g})$ was dissolved in $15 \mathrm{~mL}$ of toluene. The mixture was stirred in an oil bath at $120{ }^{\circ} \mathrm{C}$ under argon flow for about 6 h. TLC showed the reaction was complete. After cooling to room temperature, toluene was removed by rotary evaporation. And the residue was dried under vacuum at $25{ }^{\circ} \mathrm{C}$ for $24 \mathrm{~h}$ to afford $\boldsymbol{p}$ - $\mathbf{C}_{4}$-mal as colorless oil liquid (0.37 g, yield: $90 \%)$.

p-C $\mathbf{C}_{4}-\mathbf{S H}: \boldsymbol{p}-\mathbf{C}_{4}(0.07 \mathrm{mmol}, 0.41 \mathrm{~g})$ was added to a $50 \mathrm{~mL}$ round-bottom flask and dissolved in $20 \mathrm{~mL}$ of $\mathrm{MeOH} / \mathrm{CH}_{2} \mathrm{Cl}_{2}$ 1:2 mixed solvent. The solution was cooled to $-78^{\circ} \mathrm{C}$ and string for 10 minutes. With string, the acetyl chloride $(4.0 \mathrm{~mL}, 30 \mathrm{mmol})$ was added dropwise to the solution. The solution was further stirred at ambient temperature for $12 \mathrm{~h}$. After reaction, the mixture was poured into $100 \mathrm{~mL}$ deionized water and extracted with DCM. The organic layer was washed with deionized water three times and dried with anhydrous $\mathrm{Na}_{2} \mathrm{SO}_{4}$. The solvent was removed by rotary evaporation and final product $\boldsymbol{p}-\mathbf{C}_{\mathbf{4}}-\mathbf{S H}$ was obtained as colorless oil liquid (0.35 g, yield: $86 \%)$.

p-C $\mathbf{8}$ : $\boldsymbol{p}$ - $\mathbf{C}_{\mathbf{4}}$-mal $(0.37 \mathrm{~g})$ and $\boldsymbol{p}$ - $\mathbf{C}_{\mathbf{4}}-\mathbf{S H}(0.35 \mathrm{~g})$ were dissolved in $15 \mathrm{~mL}$ of dry $\mathrm{CHCl}_{3}$ in a $25 \mathrm{~mL}$ two-neck round-bottom flask equipped with a $5 \mathrm{~mL}$ slow-addition apparatus under argon atmosphere at $25^{\circ} \mathrm{C}$. TEA $(0.2 \mathrm{~mL}, 1.5 \mathrm{mmol})$ was added dropwise to the solution and the mixture was stirred for about $24 \mathrm{~h}$. The reaction mixture was quenched with $20 \mathrm{~mL}$ water and washed with $30 \mathrm{~mL}$ saturated $\mathrm{NaHCO}_{3}$ (aq.). The 
combined organic layer was dried with anhydrous $\mathrm{Na}_{2} \mathrm{SO}_{4}$ and the solvent was evaporated to afford the crude product which was purified by recycling preparative SEC to give the $\boldsymbol{p}$ - $\mathbf{C}_{\mathbf{8}}$ as colorless oil liquid. p-C8 $\left(0.32\right.$ g, yield: 45\%). ${ }^{1} \mathrm{H}$ NMR (500 MHz, $\left.\mathrm{CDCl}_{3}, \mathrm{ppm}, \delta\right): 6.51$ (s, 2H, $\left.-\mathrm{C} \underline{\mathrm{H}}=\mathrm{C} \underline{\mathrm{H}}-\right), 5.26$ (t, $J=1.0 \mathrm{~Hz}$, $2 \mathrm{H},-\mathrm{C} \underline{\mathrm{H}}(-\mathrm{O}-) \mathrm{C}-), 3.74\left(\mathrm{dd}, J=9.5,3.1 \mathrm{~Hz}, 7 \mathrm{H},-\mathrm{SC} \underline{\mathrm{H}}(\mathrm{C}=\mathrm{O}) \mathrm{CH}_{2}-\right), 3.55$ (dt, $J=13.1,7.6 \mathrm{~Hz}, 16 \mathrm{H}$, $\left.-\mathrm{NC}_{2} \mathrm{CH}_{2}-\right), 3.14,3.05\left(\mathrm{~m}, 14 \mathrm{H},-\mathrm{CHC}_{2}(\mathrm{C}=\mathrm{O})-\right), 2.94\left(\mathrm{t}, J=7.0 \mathrm{~Hz}, 2 \mathrm{H}, \mathrm{CH}_{3}(\mathrm{O}=\mathrm{C}) \mathrm{SC}_{2} \mathrm{CH}_{2}-\right), 2.84(\mathrm{~s}$, $2 \mathrm{H},-\mathrm{C} \underline{\mathrm{H}}(-\mathrm{C}=\mathrm{O}) \mathrm{C} \underline{\mathrm{H}}-), 2.47-2.63\left(\mathrm{~m}, 270 \mathrm{H},-\mathrm{C}_{2} \mathrm{SCH}_{2}-,-\underline{\mathrm{C}}_{2} \mathrm{SCH}-\right), 2.34\left(\mathrm{~s}, 3 \mathrm{H}, \mathrm{CH}_{3}(\mathrm{O}=\mathrm{C}-) \mathrm{SCH}_{2} \mathrm{CH}_{2}-\right)$, $1.85\left(\mathrm{~m}, 32 \mathrm{H},-\mathrm{CH}_{2} \mathrm{CH}_{2} \mathrm{CH}_{2} \mathrm{~S}-\right), 1.53\left(\mathrm{~m}, 96 \mathrm{H},-\mathrm{CH}_{2} \mathrm{CH}_{2} \mathrm{CH}_{3}\right), 1.44\left(\mathrm{~m}, 96 \mathrm{H},-\mathrm{CH}_{2} \mathrm{CH}_{2} \mathrm{CH}_{3}\right), 1.03(\mathrm{~m}, 128 \mathrm{H}$, $\left.-\mathrm{SiC}_{2} \mathrm{CH}_{2}-\right), 0.94\left(\mathrm{~m}, 144 \mathrm{H},-\mathrm{CH}_{2} \mathrm{CH}_{2} \underline{\mathrm{C}}_{3}\right)$.

\section{d) Synthesis of $p-, m-, o-C_{n}$}

$\boldsymbol{p}$ - $\mathbf{C}_{\boldsymbol{n}}$ with other $n$ was synthesized with appropriate precursors following the same protocol. $\mathbf{C}_{\boldsymbol{n}}$ with different regio-configurations $\left(\boldsymbol{p}-, \boldsymbol{m}-, \boldsymbol{o}-\mathbf{C}_{\boldsymbol{n}}\right)$ was synthesized with corresponding monomers following the same protocol.

\subsection{Synthesis of discrete amphiphilic giant polymeric chains}

\section{a) Synthesis of Protected V ${ }_{7}$ POSS-SH}

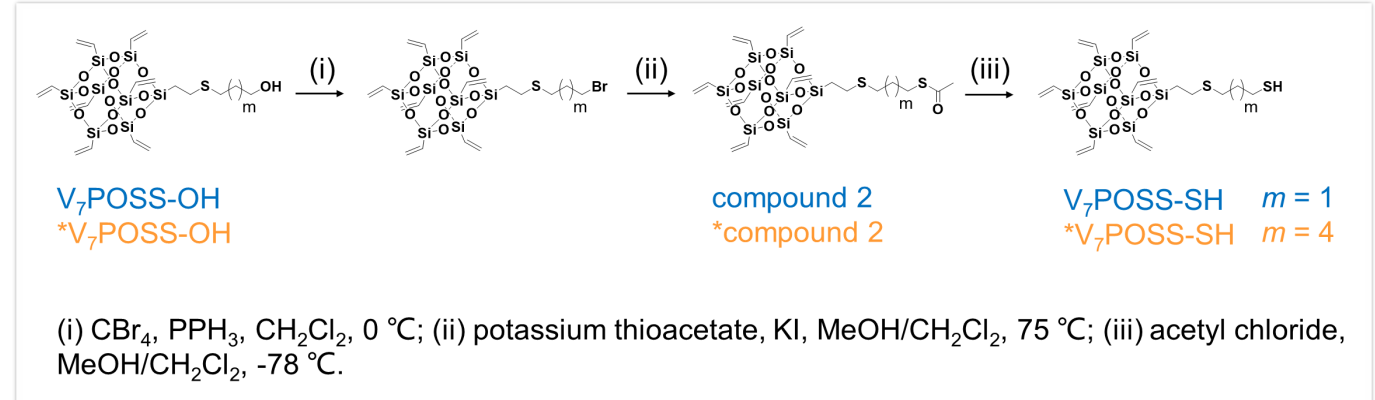

General methods for synthesizing protected V7POSS-SH: V7POSS-OH (3.63 g, 5.0 mmol) and $\mathrm{CBr}_{4}(3.36$ g, $10.0 \mathrm{mmol})$ were dissolved in anhydrous $\mathrm{CH}_{2} \mathrm{Cl}_{2}(50 \mathrm{~mL})$ in a $100 \mathrm{~mL}$ round-bottom flask. The mixture was cooled to $0{ }^{\circ} \mathrm{C}$, and $\mathrm{PPh}_{3}(2.62 \mathrm{~g}, 10.0 \mathrm{mmol})$ was added slowly into the mixture in $30 \mathrm{~min}$. The mixture was then stirred at room temperature. After string for 2 hours, $20 \mathrm{~mL}$ of $\mathrm{MeOH}, \mathrm{KI}(20 \mathrm{mg})$, and potassium thioacetate $(0.23 \mathrm{~g}, 2.0 \mathrm{mmol})$ were added, and the mixture was further stirred at $75{ }^{\circ} \mathrm{C}$ for $12 \mathrm{~h}$ under argon atmosphere. The solvent was then removed, and the crude product was purified by silica gel column chromatography with PE/DCM (10/1, v/v) as eluent to afford protected $\mathbf{V}_{7} \mathbf{P O S S}-\mathbf{S H}$ as a white powder (3.56 g, yield: $91 \%)$. 
General methods for synthesizing V7POSS-SH: Protected V ${ }_{7}$ POSS-SH (3.00 g, $3.8 \mathrm{mmol}$ ) was added to a $250 \mathrm{~mL}$ round-bottom flask and dissolved in $80 \mathrm{~mL}$ of $\mathrm{MeOH} / \mathrm{CH}_{2} \mathrm{Cl}_{2}$ (1: 2) mixed solvent. The solution was cooled to $-78^{\circ} \mathrm{C}$ and string for 10 minutes. With string, the acetyl chloride $(15.0 \mathrm{~mL}, 111 \mathrm{mmol})$ was added dropwise to the solution. The solution was further stirred at ambient temperature for $12 \mathrm{~h}$. After reaction, the mixture was poured into $100 \mathrm{~mL}$ of deionized water and extracted with DCM. The organic layer was washed with deionized water three times and dried with anhydrous $\mathrm{Na}_{2} \mathrm{SO}_{4}$. DCM was concentrated under vacuum to afford V VOSS-SH as a white powder (2.5 g, yield: $85 \%)$.

\section{c) Synthesis of $C_{n} V$ and ${ }^{*} C_{n} V$}

General methods for synthesizing $\mathbf{C}_{n} \mathbf{V}$ and ${ }^{*} \mathbf{C}_{n} \mathbf{V}$ (take $p-\mathbf{C}_{n} \mathbf{V}$ as an example): The de-protection of the

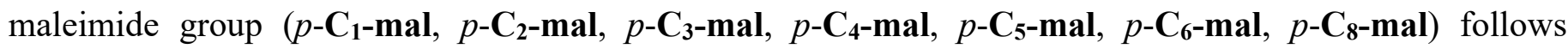
similar approach as mentioned above. $p$ - $\mathbf{C}_{n}$-mal $(200 \mathrm{mg}, n=1,2,3,4,5,6,8)$ and $\mathbf{V}_{7}$ POSS-SH (3 eq.) were dissolved in $10 \mathrm{~mL}$ of dry $\mathrm{CHCl}_{3}$ in a $25 \mathrm{~mL}$ two-neck round-bottom flask. TEA $(0.1 \mathrm{~mL}, 0.73 \mathrm{mmol})$ was added dropwise to the solution and the mixture was stirred for about $24 \mathrm{~h}$. The reaction mixture was quenched with $15 \mathrm{~mL}$ of water and washed with $10 \mathrm{~mL}$ of saturated $\mathrm{NaHCO}_{3}$ (aq.). The combined organic layer was dried with anhydrous $\mathrm{Na}_{2} \mathrm{SO}_{4}$ and the solvent was evaporated to afford the crude product which was purified by recycling preparative SEC to give the $p-\mathbf{C}_{\mathbf{n}} \mathbf{V}$ as colorless oil liquid $(170 \mathrm{mg} \sim 180 \mathrm{mg}$, yield: $77 \% \sim 82 \%)$.

p- $\mathbf{C}_{1} \mathbf{V}\left(180 \mathrm{mg}\right.$, yield: 82\%). ${ }^{1} \mathrm{H}$ NMR (500 MHz, $\left.\mathrm{CDCl}_{3}, \mathrm{ppm}, \delta\right): 5.89-6.14$ (m, 21H, $\left.\underline{\mathrm{C}}_{2}=\mathrm{C} \underline{\mathrm{H}}-\right)$ ), 3.73 (dd, $\left.J=9.0,3.6 \mathrm{~Hz}, 1 \mathrm{H},-\mathrm{SC} \underline{\mathrm{H}}(\mathrm{C}=\mathrm{O}) \mathrm{CH}_{2}-\right), 3.63\left(\mathrm{t}, J=7.2 \mathrm{~Hz}, 2 \mathrm{H},-\mathrm{NC}_{2} \mathrm{CH}_{2}-\right), 3.14,3.05(\mathrm{~m}, 2 \mathrm{H}$, $\left.-{ }^{-}{ }^{-} \underline{H}_{2}(\mathrm{C}=\mathrm{O})-\right), 2.98\left(\mathrm{t}, J=7.2 \mathrm{~Hz}, 2 \mathrm{H}, \mathrm{CH}_{3}(\mathrm{O}=\mathrm{C}) \mathrm{SC}_{2} \mathrm{CH}_{2}-\right), 2.51-2.65\left(\mathrm{~m}, 38 \mathrm{H},-\underline{\mathrm{C}}_{2} \mathrm{SC}_{2}-\right.$, $\left.-\mathrm{CH}_{2} \mathrm{SCH}-\right), 2.35$ (s, 3H, $\left.\underline{\mathrm{CH}}_{3}(\mathrm{O}=\mathrm{C}-) \mathrm{SCH}_{2} \mathrm{CH}_{2}-\right), 1.86\left(\mathrm{~m}, 6 \mathrm{H},-\mathrm{CH}_{2} \mathrm{CH}_{2} \mathrm{CH}_{2} \mathrm{~S}-\right), 1.57 \quad(\mathrm{~m}, 12 \mathrm{H}$, $\left.-\mathrm{C}_{2} \mathrm{CH}_{2} \mathrm{CH}_{3}\right), 1.43\left(\mathrm{~m}, 12 \mathrm{H},-\mathrm{CH}_{2} \mathrm{CH}_{2} \mathrm{CH}_{3}\right), 1.03\left(\mathrm{~m}, 18 \mathrm{H},-\mathrm{SiC}_{2} \mathrm{CH}_{2}-\right), 0.93$ (t, $J=7.2 \mathrm{~Hz}, 18 \mathrm{H}$, $\left.-\mathrm{CH}_{2} \mathrm{CH}_{2} \mathrm{CH}_{3}\right)$.

p- $\mathbf{C}_{2} \mathbf{V}\left(178 \mathrm{mg}\right.$, yield: 81\%). ${ }^{1} \mathrm{H}$ NMR (500 MHz, $\left.\mathrm{CDCl}_{3}, \mathrm{ppm}, \delta\right): 5.85-6.15$ (m, 21H, $\left.\underline{\mathrm{C}}_{2}=\mathrm{C} \underline{\mathrm{H}}-\right)$ ), 3.74 (dd, $\left.J=9.0,3.6 \mathrm{~Hz}, 2 \mathrm{H},-\mathrm{SC} \underline{\mathrm{H}}(\mathrm{C}=\mathrm{O}) \mathrm{CH}_{2}-\right), 3.64\left(\mathrm{t}, J=7.2 \mathrm{~Hz}, 4 \mathrm{H},-\mathrm{NC}_{2} \mathrm{CH}_{2}-\right), 3.14,3.05(\mathrm{~m}, 4 \mathrm{H}$, $\left.-\mathrm{CHC}_{2}(\mathrm{C}=\mathrm{O})-\right), 2.96\left(\mathrm{t}, J=7.2 \mathrm{~Hz}, 2 \mathrm{H}, \mathrm{CH}_{3}(\mathrm{O}=\mathrm{C}) \mathrm{SC}_{2} \mathrm{CH}_{2}-\right), 2.51-2.65\left(\mathrm{~m}, 72 \mathrm{H},-\mathrm{C}_{2} \mathrm{SC}_{2}-{ }_{2}^{-}\right.$, $\left.-\underline{\mathrm{CH}}_{2} \mathrm{SCH}-\right), 2.34\left(\mathrm{~s}, 3 \mathrm{H}, \mathrm{C}_{3}(\mathrm{O}=\mathrm{C}-) \mathrm{SCH}_{2} \mathrm{CH}_{2}-\right), 1.85\left(\mathrm{~m}, 10 \mathrm{H},-\mathrm{CH}_{2} \mathrm{CH}_{2} \mathrm{CH}_{2} \mathrm{~S}-\right), 1.53 \quad(\mathrm{~m}, 24 \mathrm{H}$, $\left.-\underline{\mathrm{C}}_{2} \mathrm{CH}_{2} \mathrm{CH}_{3}\right), 1.45\left(\mathrm{~m}, 24 \mathrm{H},-\mathrm{CH}_{2} \mathrm{C}_{2} \mathrm{CH}_{3}\right), 1.04\left(\mathrm{~m}, 34 \mathrm{H},-\mathrm{SiC}_{2} \mathrm{CH}_{2}-\right), 0.96$ (t, $J=7.2 \mathrm{~Hz}, 36 \mathrm{H}$, $\left.-\mathrm{CH}_{2} \mathrm{CH}_{2} \mathrm{CH}_{3}\right)$.

p-C $\mathbf{3}$ V (176 mg, yield: 81\%). ${ }^{1} \mathrm{H}$ NMR (500 MHz, $\left.\mathrm{CDCl}_{3}, \mathrm{ppm}, \delta\right)$ : 5.83-6.13 (m, 21H, $\left.\underline{\mathrm{C}}_{2}=\mathrm{C} \underline{\mathrm{H}}-\right)$, 3.72 (dd, $\left.J=9.0,3.3 \mathrm{~Hz}, 3 \mathrm{H},-\mathrm{SC} \underline{\mathrm{H}}(\mathrm{C}=\mathrm{O}) \mathrm{CH}_{2}-\right), 3.67\left(\mathrm{t}, J=7.2 \mathrm{~Hz}, 6 \mathrm{H},-\mathrm{NC}_{2} \mathrm{CH}_{2}-\right), 3.14,3.05(\mathrm{~m}, 6 \mathrm{H}$, 
$\left.-\mathrm{CHC}_{2}(\mathrm{C}=\mathrm{O})-\right), 2.92$ (t, $\left.J=7.2 \mathrm{~Hz}, 2 \mathrm{H}, \mathrm{CH}_{3}(\mathrm{O}=\mathrm{C}) \mathrm{SC}_{2} \mathrm{CH}_{2}-\right), 2.51-2.65\left(\mathrm{~m}, 106 \mathrm{H},-\mathrm{C}_{2} \mathrm{SC}_{2}{ }^{-}\right.$, $\left.-\mathrm{CH}_{2} \mathrm{SCH}-\right), 2.36$ (s, 3H, $\left.\mathrm{CH}_{3}(\mathrm{O}=\mathrm{C}-) \mathrm{SCH}_{2} \mathrm{CH}_{2}-\right), 1.84\left(\mathrm{~m}, 14 \mathrm{H},-\mathrm{CH}_{2} \mathrm{CH}_{2} \mathrm{CH}_{2} \mathrm{~S}-\right), 1.54 \quad(\mathrm{~m}, 36 \mathrm{H}$, $\left.-\mathrm{C}_{2} \mathrm{CH}_{2} \mathrm{CH}_{3}\right), 1.47\left(\mathrm{~m}, 36 \mathrm{H},-\mathrm{CH}_{2} \underline{\mathrm{C}}_{2} \mathrm{CH}_{3}\right), 1.03\left(\mathrm{~m}, 50 \mathrm{H},-\mathrm{SiC}_{2} \mathrm{CH}_{2}-\right), 0.94$ (t, $J=7.4 \mathrm{~Hz}, 54 \mathrm{H}$, $-\mathrm{CH}_{2} \mathrm{CH}_{2} \mathrm{CH}_{3}$ ).

p-C $\mathbf{C}_{4} \mathbf{V}\left(175 \mathrm{mg}\right.$, yield: 80\%). ${ }^{1} \mathrm{H}$ NMR (500 MHz, $\left.\mathrm{CDCl}_{3}, \mathrm{ppm}, \delta\right)$ : 5.83-6.15 (m, 21H, $\left.\underline{\mathrm{C}}_{2}=\mathrm{C} \underline{\mathrm{H}}-\right)$, 3.71 (dd, $\left.J=9.0,3.5 \mathrm{~Hz}, 4 \mathrm{H},-\mathrm{SC} \underline{\mathrm{H}}(\mathrm{C}=\mathrm{O}) \mathrm{CH}_{2}-\right), 3.62\left(\mathrm{t}, J=7.8 \mathrm{~Hz}, 8 \mathrm{H},-\mathrm{NC}_{2} \mathrm{CH}_{2}-\right), 3.14,3.05(\mathrm{~m}, 8 \mathrm{H}$, $\left.-\mathrm{CHC}_{2}(\mathrm{C}=\mathrm{O})-\right), 2.99\left(\mathrm{t}, J=7.5 \mathrm{~Hz}, 2 \mathrm{H}, \mathrm{CH}_{3}(\mathrm{O}=\mathrm{C}) \mathrm{SC}_{\mathrm{H}_{2}} \mathrm{CH}_{2}-\right), 2.51-2.65\left(\mathrm{~m}, 140 \mathrm{H},-\mathrm{C}_{2} \mathrm{SC}_{2}-\underline{H}_{2}\right.$, $\left.-\underline{\mathrm{H}}_{2} \mathrm{SCH}-\right), 2.32$ (s, 3H, $\left.\underline{\mathrm{C}}_{3}(\mathrm{O}=\mathrm{C}-) \mathrm{SCH}_{2} \mathrm{CH}_{2}-\right), 1.83\left(\mathrm{~m}, 18 \mathrm{H},-\mathrm{CH}_{2} \mathrm{C}_{2} \mathrm{CH}_{2} \mathrm{~S}-\right), 1.52 \quad(\mathrm{~m}, 48 \mathrm{H}$, $\left.-\mathrm{C}_{2} \mathrm{CH}_{2} \mathrm{CH}_{3}\right), 1.44\left(\mathrm{~m}, 48 \mathrm{H},-\mathrm{CH}_{2} \underline{\mathrm{C}}_{2} \mathrm{CH}_{3}\right), 1.03\left(\mathrm{~m}, 66 \mathrm{H},-\mathrm{SiC}_{2} \mathrm{CH}_{2}-\right), 0.92$ (t, $J=7.6 \mathrm{~Hz}, 72 \mathrm{H}$, $\left.-\mathrm{CH}_{2} \mathrm{CH}_{2} \mathrm{CH}_{3}\right)$.

p-C5V (172 mg, yield: 78\%). ${ }^{1} \mathrm{H}$ NMR (500 MHz, $\left.\mathrm{CDCl}_{3}, \mathrm{ppm}, \delta\right): 5.81-6.14$ (m, 21H, $\left.\mathrm{CH}_{2}=\mathrm{C} \underline{\mathrm{H}}-\right)$, 3.70 (dd, $\left.J=9.3,3.6 \mathrm{~Hz}, 5 \mathrm{H},-\mathrm{SC} \underline{\mathrm{H}}(\mathrm{C}=\mathrm{O}) \mathrm{CH}_{2}-\right), 3.62\left(\mathrm{t}, J=7.1 \mathrm{~Hz}, 10 \mathrm{H},-\mathrm{NC}_{2} \mathrm{CH}_{2}\right), 3.14,3.05(\mathrm{~m}, 10 \mathrm{H}$, $\left.-\mathrm{CHC}_{2}(\mathrm{C}=\mathrm{O})-\right), 2.96\left(\mathrm{t}, J=7.4 \mathrm{~Hz}, 2 \mathrm{H}, \mathrm{CH}_{3}(\mathrm{O}=\mathrm{C}) \mathrm{SC}_{\mathrm{H}_{2}} \mathrm{CH}_{2}-\right), 2.51-2.65\left(\mathrm{~m}, 174 \mathrm{H},-\underline{\mathrm{C}}_{2} \mathrm{SC}_{2}-\underline{\mathrm{H}}_{2}\right.$, $\left.-\underline{\mathrm{C}}_{2} \mathrm{SCH}-\right), 2.31$ (s, 3H, $\left.\underline{\mathrm{C}}_{3}(\mathrm{O}=\mathrm{C}-) \mathrm{SCH}_{2} \mathrm{CH}_{2}-\right), 1.81\left(\mathrm{~m}, 22 \mathrm{H},-\mathrm{CH}_{2} \underline{\mathrm{C}}_{2} \mathrm{CH}_{2} \mathrm{~S}-\right), 1.52 \quad(\mathrm{~m}, 60 \mathrm{H}$, $\left.-\mathrm{CH}_{2} \mathrm{CH}_{2} \mathrm{CH}_{3}\right), 1.43\left(\mathrm{~m}, 60 \mathrm{H},-\mathrm{CH}_{2} \mathrm{CH}_{2} \mathrm{CH}_{3}\right), 1.06\left(\mathrm{~m}, 82 \mathrm{H},-\mathrm{SiC}_{2} \mathrm{CH}_{2}-\right), 0.91$ (t, $J=7.2 \mathrm{~Hz}, 90 \mathrm{H}$, $\left.-\mathrm{CH}_{2} \mathrm{CH}_{2} \mathrm{CH}_{3}\right)$.

p-C $\mathbf{C} \mathbf{V}\left(170 \mathrm{mg}\right.$, yield: 77\%). ${ }^{1} \mathrm{H}$ NMR (500 MHz, $\left.\mathrm{CDCl}_{3}, \mathrm{ppm}, \delta\right)$ : 5.87-6.18 (m, 21H, $\left.\underline{\mathrm{C}}_{2}=\mathrm{C} \underline{\mathrm{H}}-\right)$, 3.73 (dd, $\left.J=9.2,3.6 \mathrm{~Hz}, 6 \mathrm{H},-\mathrm{SC} \underline{\mathrm{H}}(\mathrm{C}=\mathrm{O}) \mathrm{CH}_{2}-\right), 3.62\left(\mathrm{t}, J=7.3 \mathrm{~Hz}, 12 \mathrm{H},-\mathrm{NC}_{2} \mathrm{CH}_{2}\right), 3.14,3.05(\mathrm{~m}, 12 \mathrm{H}$, $\left.-{ }^{-}{ } \underline{H}_{2}(\mathrm{C}=\mathrm{O})-\right), 2.92\left(\mathrm{t}, J=7.7 \mathrm{~Hz}, 2 \mathrm{H}, \mathrm{CH}_{3}(\mathrm{O}=\mathrm{C}) \mathrm{SC}_{2} \mathrm{CH}_{2}-\right), 2.51-2.65\left(\mathrm{~m}, 208 \mathrm{H},-\underline{\mathrm{C}}_{2} \mathrm{SC}_{2_{2}}^{-}\right.$, $\left.-\underline{\mathrm{C}}_{2} \mathrm{SCH}-\right), 2.39$ (s, 3H, $\left.\underline{\mathrm{H}}_{3}(\mathrm{O}=\mathrm{C}-) \mathrm{SCH}_{2} \mathrm{CH}_{2}\right), 1.82$ (m, 26H, $\left.-\mathrm{CH}_{2} \mathrm{C}_{2} \mathrm{CH}_{2} \mathrm{~S}-\right), 1.537$ (m, 72H, $\left.-\underline{\mathrm{C}}_{2} \mathrm{CH}_{2} \mathrm{CH}_{3}\right), 1.41\left(\mathrm{~m}, 72 \mathrm{H},-\mathrm{CH}_{2} \underline{\mathrm{H}}_{2} \mathrm{CH}_{3}\right), 1.06\left(\mathrm{~m}, 98 \mathrm{H},-\mathrm{SiC}_{2} \mathrm{CH}_{2}\right), 0.91$ (t, $J=7.9 \mathrm{~Hz}, 108 \mathrm{H}$, $\left.-\mathrm{CH}_{2} \mathrm{CH}_{2} \mathrm{C}_{3}\right)$.

p-C $\mathbf{C}_{8} \mathbf{V}\left(170 \mathrm{mg}\right.$, yield: 77\%). ${ }^{1} \mathrm{H}$ NMR (500 MHz, $\left.\mathrm{CDCl}_{3}, \mathrm{ppm}, \delta\right): 5.81-6.11$ (m, 21H, $\left.\underline{\mathrm{C}}_{2}=\mathrm{C} \underline{\mathrm{H}}-\right)$, 3.72 (dd, $\left.J=9.3,3.6 \mathrm{~Hz}, 8 \mathrm{H},-\mathrm{SC} \underline{\mathrm{H}}(\mathrm{C}=\mathrm{O}) \mathrm{CH}_{2}-\right), 3.62\left(\mathrm{t}, J=7.5 \mathrm{~Hz}, 16 \mathrm{H},-\mathrm{NC}_{\mathrm{H}_{2}} \mathrm{CH}_{2}-\right), 3.14,3.05(\mathrm{~m}, 16 \mathrm{H}$, $\left.-\mathrm{CHC}_{2}(\mathrm{C}=\mathrm{O})-\right), 2.92\left(\mathrm{t}, J=7.1 \mathrm{~Hz}, 2 \mathrm{H}, \mathrm{CH}_{3}(\mathrm{O}=\mathrm{C}) \mathrm{SC}_{2} \mathrm{CH}_{2}-\right), 2.51-2.65\left(\mathrm{~m}, 276 \mathrm{H},-\underline{C}_{2} \mathrm{SC}_{2}-\right.$, $\left.-\underline{\mathrm{H}}_{2} \mathrm{SCH}-\right), 2.33$ (s, 3H, $\left.\mathrm{CH}_{3}(\mathrm{O}=\mathrm{C}-) \mathrm{SCH}_{2} \mathrm{CH}_{2}-\right), 1.86\left(\mathrm{~m}, 34 \mathrm{H},-\mathrm{CH}_{2} \mathrm{CH}_{2} \mathrm{CH}_{2} \mathrm{~S}-\right), 1.54 \quad(\mathrm{~m}, 96 \mathrm{H}$, $\left.-\mathrm{C}_{2} \mathrm{CH}_{2} \mathrm{CH}_{3}\right), 1.43\left(\mathrm{~m}, 96 \mathrm{H},-\mathrm{CH}_{2} \underline{\mathrm{C}}_{2} \mathrm{CH}_{3}\right), 1.04\left(\mathrm{~m}, 130 \mathrm{H},-\mathrm{SiC}_{2} \mathrm{CH}_{2}-\right), 0.93$ (t, $J=7.3 \mathrm{~Hz}, 144 \mathrm{H}$, $\left.-\mathrm{CH}_{2} \mathrm{CH}_{2} \mathrm{C}_{3}\right)$.

\section{d) Synthesis of $C_{n} D$ and $* C_{n} D$}

General methods for synthesizing $\mathbf{C}_{n} \mathbf{D}$ and ${ }^{*} \mathbf{C}_{\boldsymbol{n}} \mathbf{D}$ (take $\boldsymbol{p}-\mathbf{C}_{\boldsymbol{n}} \mathbf{D}$ as an example): $\boldsymbol{p}-\mathbf{C}_{\mathbf{n}} \mathbf{V}(200 \mathrm{mg}), 2$ eq. of 1-thioglycerol to vinyl group, and 0.03 eq of photo-initiator DMPA were dissolved in $4 \mathrm{~mL}$ of THF. The 
solution was illuminated under $365 \mathrm{~nm}$ UV light in a UV reactor for $35 \mathrm{~min}$. The solution is concentrated and purified by recycling preparative SEC to give the $\boldsymbol{p}-\mathbf{C}_{\mathbf{n}} \mathbf{D}$ as colorless oil liquid.

p-C $\mathbf{1}$ D (153 mg, yield: 82\%). ${ }^{1} \mathrm{H}$ NMR (500 MHz, $\left.\mathrm{CDCl}_{3}, \mathrm{ppm}, \delta\right): 3.71-3.82(\mathrm{~m}, 22 \mathrm{H},-\mathrm{C} \underline{\mathrm{H}}(\mathrm{OH}) \mathrm{C} \underline{\mathrm{H}} 2 \mathrm{OH}$, $\left.-\mathrm{SC} \underline{\mathrm{H}}(\mathrm{C}=\mathrm{O}) \mathrm{CH}_{2}-\right), 3.57\left(\mathrm{t}, J=7.0 \mathrm{~Hz}, 2 \mathrm{H},-\mathrm{NC}_{2} \mathrm{CH}_{2}-\right), 3.14,3.05\left(\mathrm{~m}, 2 \mathrm{H},-\mathrm{CHC}_{2}(\mathrm{C}=\mathrm{O})-\right), 2.96(\mathrm{t}, J=$ $\left.7.3 \mathrm{~Hz}, \quad 2 \mathrm{H}, \quad \mathrm{CH}_{3}(\mathrm{O}=\mathrm{C}) \mathrm{SC}_{2} \mathrm{CH}_{2}-\right), 2.51-2.72\left(\mathrm{~m}, 66 \mathrm{H},-\mathrm{C}_{2} \mathrm{SC}_{2_{2}}, \quad-\mathrm{C}_{2} \mathrm{SCH}-\right), 2.35 \quad(\mathrm{~s}, \quad 3 \mathrm{H}$, $\left.\mathrm{CH}_{3}(\mathrm{O}=\mathrm{C}-) \mathrm{SCH}_{2} \mathrm{CH}_{2}-\right), 1.86\left(\mathrm{~m}, 6 \mathrm{H},-\mathrm{CH}_{2} \mathrm{CH}_{2} \mathrm{CH}_{2} \mathrm{~S}-\right), 1.57\left(\mathrm{~m}, 12 \mathrm{H},-\mathrm{CH}_{2} \mathrm{CH}_{2} \mathrm{CH}_{3}\right), 1.43(\mathrm{~m}, 12 \mathrm{H}$, $\left.-\mathrm{CH}_{2} \mathrm{CH}_{2} \mathrm{CH}_{3}\right), 1.04\left(\mathrm{~m}, 32 \mathrm{H},-\mathrm{SiCH}_{2} \mathrm{CH}_{2}-\right), 0.93\left(\mathrm{t}, J=7.2 \mathrm{~Hz}, 18 \mathrm{H},-\mathrm{CH}_{2} \mathrm{CH}_{2} \mathrm{CH}_{3}\right)$.

p- $\mathbf{C}_{2} \mathbf{D}\left(142 \mathrm{mg}\right.$, yield: 80\%). ${ }^{1} \mathrm{H}-\mathrm{NMR}\left(500 \mathrm{MHz}, \mathrm{CDCl}_{3}, \mathrm{ppm}, \delta\right): 3.70-3.82\left(\mathrm{~m}, 23 \mathrm{H},-\mathrm{CH}(\mathrm{OH}) \underline{\mathrm{H}}_{2} \mathrm{OH}\right.$, $\left.-\mathrm{SC} \underline{\mathrm{H}}(\mathrm{C}=\mathrm{O}) \mathrm{CH}_{2}-\right), 3.58\left(\mathrm{t}, J=7.1 \mathrm{~Hz}, 4 \mathrm{H},-\mathrm{NC}_{2} \mathrm{CH}_{2}-\right), 3.13,3.05\left(\mathrm{~m}, 4 \mathrm{H},-\mathrm{CHCH}_{2}(\mathrm{C}=\mathrm{O})-\right), 2.98(\mathrm{t}, J=$ $\left.7.4 \mathrm{~Hz}, 2 \mathrm{H}, \quad \mathrm{CH}_{3}(\mathrm{O}=\mathrm{C}) \mathrm{SC}_{2} \mathrm{CH}_{2}\right), 2.51-2.65$ (m, 100H, - $\left.\underline{\mathrm{H}}_{2} \mathrm{SC}_{\underline{H}_{2}}, \quad-\mathrm{C}_{2} \mathrm{SCH}-\right), 2.35 \quad(\mathrm{~s}, \quad 3 \mathrm{H}$, $\left.\mathrm{C}_{3}(\mathrm{O}=\mathrm{C}-) \mathrm{SCH}_{2} \mathrm{CH}_{2}-\right), 1.87\left(\mathrm{~m}, 10 \mathrm{H},-\mathrm{CH}_{2} \mathrm{CH}_{2} \mathrm{CH}_{2} \mathrm{~S}-\right), 1.57\left(\mathrm{~m}, 24 \mathrm{H},-\mathrm{CH}_{2} \mathrm{CH}_{2} \mathrm{CH}_{3}\right), 1.44(\mathrm{~m}, 24 \mathrm{H}$, $\left.-\mathrm{CH}_{2} \underline{\mathrm{C}}_{2} \mathrm{CH}_{3}\right), 1.03\left(\mathrm{~m}, 48 \mathrm{H},-\mathrm{SiCH}_{2} \mathrm{CH}_{2}-\right), 0.93\left(\mathrm{t}, J=7.3 \mathrm{~Hz}, 36 \mathrm{H},-\mathrm{CH}_{2} \mathrm{CH}_{2} \mathrm{CH}_{3}\right)$.

p-C 3 D (139 mg, yield: 78\%). ${ }^{1} \mathrm{H}$ NMR $\left.500 \mathrm{MHz}, \mathrm{CDCl}_{3}, \mathrm{ppm}, \delta\right): 3.61-3.86\left(\mathrm{~m}, 24 \mathrm{H},-\mathrm{C} \underline{\mathrm{H}}(\mathrm{OH}) \underline{\mathrm{H}}_{2} \mathrm{OH}\right.$, $\left.-\mathrm{SC} \underline{\mathrm{H}}(\mathrm{C}=\mathrm{O}) \mathrm{CH}_{2}-\right), 3.61$ (t, $\left.J=7.8 \mathrm{~Hz}, 6 \mathrm{H},-\mathrm{NC}_{2} \mathrm{CH}_{2}-\right), 3.14,3.05\left(\mathrm{~m}, 6 \mathrm{~m}, \mathrm{H},-\mathrm{CHC}_{2}(\mathrm{C}=\mathrm{O})-\right), 2.99$ (t, $J=$ $\left.7.7 \mathrm{~Hz}, 2 \mathrm{H}, \quad \mathrm{CH}_{3}(\mathrm{O}=\mathrm{C}) \mathrm{SC}_{2} \mathrm{CH}_{2}-\right), 2.51-2.63$ (m, 134H, $\left.-\underline{\mathrm{C}}_{2} \mathrm{SC}_{2} 2_{2},-\underline{\mathrm{C}}_{2} \mathrm{SCH}-\right), 2.32$ (s, 3H, $\left.\mathrm{CH}_{3}(\mathrm{O}=\mathrm{C}-) \mathrm{SCH}_{2} \mathrm{CH}_{2}-\right), 1.87\left(\mathrm{~m}, 14 \mathrm{H},-\mathrm{CH}_{2} \mathrm{CH}_{2} \mathrm{CH}_{2} \mathrm{~S}-\right), 1.53\left(\mathrm{~m}, 36 \mathrm{H},-\mathrm{CH}_{2} \mathrm{CH}_{2} \mathrm{CH}_{3}\right), 1.47(\mathrm{~m}, 36 \mathrm{H}$, $\left.-\mathrm{CH}_{2} \mathrm{CH}_{2} \mathrm{CH}_{3}\right), 1.04\left(\mathrm{~m}, 64 \mathrm{H},-\mathrm{SiCH}_{2} \mathrm{CH}_{2}-\right), 0.91$ (t, $\left.J=7.7 \mathrm{~Hz}, 54 \mathrm{H},-\mathrm{CH}_{2} \mathrm{CH}_{2} \mathrm{CH}_{3}\right)$.

p-C $\mathbf{C}_{4} \mathbf{D}\left(137 \mathrm{mg}\right.$, yield: 77\%). ${ }^{1} \mathrm{H}$ NMR (500 MHz, $\left.\mathrm{CDCl}_{3}, \mathrm{ppm}, \delta\right): 3.67-3.81\left(\mathrm{~m}, 25 \mathrm{H},-\mathrm{C} \underline{\mathrm{H}}(\mathrm{OH}) \underline{\mathrm{H}}_{2} \mathrm{OH}\right.$, $\left.-\mathrm{SC} \underline{\mathrm{H}}(\mathrm{C}=\mathrm{O}) \mathrm{CH}_{2}-\right), 3.61\left(\mathrm{t}, J=7.4 \mathrm{~Hz}, 8 \mathrm{H},-\mathrm{NC}_{2} \mathrm{CH}_{2}-\right), 3.14,3.05\left(\mathrm{~m}, 8 \mathrm{H},-\mathrm{CHC}_{2}(\mathrm{C}=\mathrm{O})-\right), 2.92(\mathrm{t}, J=$ $\left.7.5 \mathrm{~Hz}, 2 \mathrm{H}, \quad \mathrm{CH}_{3}(\mathrm{O}=\mathrm{C}) \mathrm{SC}_{2} \mathrm{CH}_{2}\right), \quad 2.51-2.65 \quad\left(\mathrm{~m}, \quad 168 \mathrm{H}, \quad-\underline{\mathrm{C}}_{2} \mathrm{SC}_{\underline{H}_{2}}, \quad-\mathrm{C}_{2} \mathrm{SCH}-\right), \quad 2.39 \quad(\mathrm{~s}, \quad 3 \mathrm{H}$, $\left.\mathrm{C}_{3}(\mathrm{O}=\mathrm{C}-) \mathrm{SCH}_{2} \mathrm{CH}_{2}-\right), 1.81\left(\mathrm{~m}, 18 \mathrm{H},-\mathrm{CH}_{2} \mathrm{CH}_{2} \mathrm{CH}_{2} \mathrm{~S}-\right), 1.54\left(\mathrm{~m}, 48 \mathrm{H},-\mathrm{CH}_{2} \mathrm{CH}_{2} \mathrm{CH}_{3}\right), 1.47(\mathrm{~m}, 48 \mathrm{H}$, $\left.-\mathrm{CH}_{2} \mathrm{CH}_{2} \mathrm{CH}_{3}\right), 1.03\left(\mathrm{~m}, 80 \mathrm{H},-\mathrm{SiCH}_{2} \mathrm{CH}_{2}-\right), 0.97$ (t, $\left.J=7.1 \mathrm{~Hz}, 72 \mathrm{H},-\mathrm{CH}_{2} \mathrm{CH}_{2} \mathrm{CH}_{3}\right)$.

p-C5 $\mathbf{D}$ (129 mg, yield: 75\%). ${ }^{1} \mathrm{H}$ NMR (500 MHz, $\left.\mathrm{CDCl}_{3}, \mathrm{ppm}, \delta\right): 3.71-3.82\left(\mathrm{~m}, 26 \mathrm{H},-\mathrm{C} \underline{\mathrm{H}}(\mathrm{OH}) \underline{\mathrm{H}}_{2} \mathrm{OH}\right.$, $\left.-\mathrm{SC} \underline{\mathrm{H}}(\mathrm{C}=\mathrm{O}) \mathrm{CH}_{2}-\right), 3.63\left(\mathrm{t}, J=7.3 \mathrm{~Hz}, 10 \mathrm{H},-\mathrm{NC}_{2} \mathrm{CH}_{2}-\right), 3.14,3.05\left(\mathrm{~m}, 10 \mathrm{H},-\mathrm{CHC}_{2}(\mathrm{C}=\mathrm{O})-\right), 2.98(\mathrm{t}, J=$ $\left.7.8 \mathrm{~Hz}, 2 \mathrm{H}, \quad \mathrm{CH}_{3}(\mathrm{O}=\mathrm{C}) \mathrm{SCH}_{2} \mathrm{CH}_{2}-\right), 2.51-2.65\left(\mathrm{~m}, 102 \mathrm{H},-\mathrm{C}_{2} \mathrm{SC}_{2}-\underline{\mathrm{H}}_{2},-\underline{\mathrm{H}}_{2} \mathrm{SCH}-\right), 2.35$ (s, 3H, $\left.\mathrm{C}_{3}(\mathrm{O}=\mathrm{C}-) \mathrm{SCH}_{2} \mathrm{CH}_{2}-\right), 1.86\left(\mathrm{~m}, 22 \mathrm{H},-\mathrm{CH}_{2} \mathrm{CH}_{2} \mathrm{CH}_{2} \mathrm{~S}-\right), 1.57\left(\mathrm{~m}, 60 \mathrm{H},-\mathrm{CH}_{2} \mathrm{CH}_{2} \mathrm{CH}_{3}\right), 1.43(\mathrm{~m}, 60 \mathrm{H}$, $\left.-\mathrm{CH}_{2} \mathrm{CH}_{2} \mathrm{CH}_{3}\right), 1.03\left(\mathrm{~m}, 96 \mathrm{H},-\mathrm{SiCH}_{2} \mathrm{CH}_{2}-\right), 0.93\left(\mathrm{t}, J=7.5 \mathrm{~Hz}, 90 \mathrm{H},-\mathrm{CH}_{2} \mathrm{CH}_{2} \mathrm{CH}_{3}\right)$.

p-C $\mathbf{6} \mathbf{D}$ (122 mg, yield: 72\%). ${ }^{1} \mathrm{H}$ NMR (500 MHz, $\left.\mathrm{CDCl}_{3}, \mathrm{ppm}, \delta\right)$ : 3.71-3.82 (m, 27H, $-\mathrm{C} \underline{\mathrm{H}}(\mathrm{OH}) \mathrm{C}_{2} \mathrm{OH}$, $\left.-\mathrm{SC} \underline{\mathrm{H}}(\mathrm{C}=\mathrm{O}) \mathrm{CH}_{2}-\right), 3.64\left(\mathrm{t}, J=7.6 \mathrm{~Hz}, 12 \mathrm{H},-\mathrm{NC}_{2} \mathrm{CH}_{2}-\right), 3.14,3.05\left(\mathrm{~m}, 12 \mathrm{H},-\mathrm{CHC}_{2}(\mathrm{C}=\mathrm{O})-\right), 2.98(\mathrm{t}, J=$ $\left.7.9 \mathrm{~Hz}, 2 \mathrm{H}, \quad \mathrm{CH}_{3}(\mathrm{O}=\mathrm{C}) \mathrm{SC}_{2} \mathrm{CH}_{2}-\right), 2.51-2.65$ (m, 136H, $\left.-\underline{\mathrm{C}}_{2} \mathrm{SC}_{2} 2_{2},-\mathrm{C}_{2} \mathrm{SCH}-\right), 2.36$ (s, 3H, $\left.\mathrm{CH}_{3}(\mathrm{O}=\mathrm{C}-) \mathrm{SCH}_{2} \mathrm{CH}_{2}-\right), 1.83\left(\mathrm{~m}, 26 \mathrm{H},-\mathrm{CH}_{2} \mathrm{CH}_{2} \mathrm{CH}_{2} \mathrm{~S}-\right), 1.55\left(\mathrm{~m}, 72 \mathrm{H},-\mathrm{CH}_{2} \mathrm{CH}_{2} \mathrm{CH}_{3}\right), 1.42(\mathrm{~m}, 72 \mathrm{H}$, 
$\left.-\mathrm{CH}_{2} \underline{\mathrm{CH}}_{2} \mathrm{CH}_{3}\right), 1.07\left(\mathrm{~m}, 112 \mathrm{H},-\mathrm{SiCH}_{2} \mathrm{CH}_{2}-\right), 0.93\left(\mathrm{t}, J=7.4 \mathrm{~Hz}, 108 \mathrm{H},-\mathrm{CH}_{2} \mathrm{CH}_{2} \mathrm{CH}_{3}\right)$.

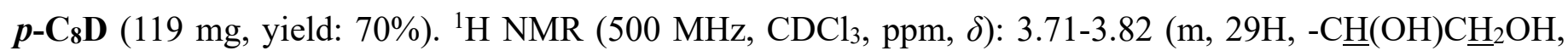
$\left.-\mathrm{SC} \underline{\mathrm{H}}(\mathrm{C}=\mathrm{O}) \mathrm{CH}_{2}-\right), 3.63\left(\mathrm{t}, J=7.3 \mathrm{~Hz}, 16 \mathrm{H},-\mathrm{NC}_{2} \mathrm{CH}_{2}-\right), 3.14,3.05\left(\mathrm{~m}, 16 \mathrm{H},-\mathrm{CHCH}_{2}(\mathrm{C}=\mathrm{O})-\right), 2.98(\mathrm{t}, J=$ $\left.7.1 \mathrm{~Hz}, 2 \mathrm{H}, \quad \mathrm{CH}_{3}(\mathrm{O}=\mathrm{C}) \mathrm{SC}_{2} \mathrm{CH}_{2}-\right), 2.51-2.65$ (m, 304H, - $\left.\underline{\mathrm{C}}_{2} \mathrm{SC}_{\mathrm{H}_{2}}-,-\underline{\mathrm{C}}_{2} \mathrm{SCH}-\right), 2.35(\mathrm{~s}, 3 \mathrm{H}$, $\left.\mathrm{C}_{3}(\mathrm{O}=\mathrm{C}-) \mathrm{SCH}_{2} \mathrm{CH}_{2}-\right), 1.86\left(\mathrm{~m}, 34 \mathrm{H},-\mathrm{CH}_{2} \mathrm{CH}_{2} \mathrm{CH}_{2} \mathrm{~S}-\right), 1.57\left(\mathrm{~m}, 96 \mathrm{H},-\mathrm{C}_{2} \mathrm{CH}_{2} \mathrm{CH}_{3}\right), 1.43(\mathrm{~m}, 96 \mathrm{H}$, $\left.-\mathrm{CH}_{2} \mathrm{CH}_{2} \mathrm{CH}_{3}\right), 1.03\left(\mathrm{~m}, 144 \mathrm{H},-\mathrm{SiCH}_{2} \mathrm{CH}_{2}-\right), 0.93\left(\mathrm{t}, J=7.0 \mathrm{~Hz}, 144 \mathrm{H},-\mathrm{CH}_{2} \mathrm{CH}_{2} \mathrm{CH}_{3}\right)$. 


\section{Equation and calculations}

\subsection{Volume fraction of CPOSS $\left(f_{\mathrm{C}}\right)$.}

The volume fraction of CPOSS $\left(f_{\mathrm{C}}\right)$ can be calculated by Eq. S1:

$$
f_{C}=\frac{n M_{C} / \rho_{C}}{n M_{C} / \rho_{C}+M_{D} / \rho_{D}}
$$

where $M_{\mathrm{D}}$ is the molecular weight of DPOSS $(1430.2 \mathrm{~g} / \mathrm{mol}), M_{\mathrm{C}}$ is the molecular weight of CPOSS (1453.6 $\mathrm{g} / \mathrm{mol}), n$ is the number of CPOSS, and $\rho_{\mathrm{D}}$ and $\rho_{\mathrm{C}}$ are the density of DPOSS and CPOSS, respectively. The density of DPOSS is measured to be around $1.43 \mathrm{~g} / \mathrm{cm}^{3}$, and the density of CPOSS is estimated to be around $1.1 \mathrm{~g} / \mathrm{cm}^{3}$ (assuming the density of CPOSS is similar to that of BPOSS, i.e., POSS with seven isobutyl groups). ${ }^{[1]}$

\subsection{Characteristic dimension of the phase (a).}

The dimension of the phase (a) refers to lamellar periodicities (for LAM), inter-column distances (for HEX), and lattice parameters (for DG and BCC). It can be calculated from domain spacing $(d)$ accordingly.

$$
\begin{array}{lc}
a=d & \text { for LAM } \\
a=\sqrt{6} d & \text { for DG } \\
a=2 d / \sqrt{3} & \text { for HEX } \\
a=\sqrt{2} d & \text { for BCC }
\end{array}
$$

\subsection{Average number of molecules $(\mu)$, fan-angle and cone-angle $(\alpha)^{[2]}$}

For HEX phase, the average number of molecules $(\mu)$ in one DPOSS thickness $(h=1.5 \mathrm{~nm})$ is:

$$
\begin{gathered}
\mu=\frac{V_{\text {cell }}}{\left(V_{C}+V_{D}\right)}=\frac{2 d^{2} h N_{A}}{\sqrt{3}\left[M_{D} / \rho_{D}+n * M_{C} / \rho_{C}\right]} \\
\alpha=2 \pi / \mu
\end{gathered}
$$


where $N_{\mathrm{A}}$ is Avogadro's constant.

For BCC phase, the average number of molecules $(\mu)$ in each unit cell can be calculated by ${ }^{[2]}$ :

$$
\mu=\frac{V_{\text {cell }}}{\left(V_{C}+V_{D}\right)}=\frac{(\sqrt{2} d)^{3} N_{A}}{2\left[M_{D} / \rho_{D}+n * M_{C} / \rho_{C}\right]}
$$

The solid angle of the cone-shape molecules $\Omega=4 \pi / \mu$.

The corresponding cone angle $\theta$ can be calculated from the equation:

$$
\begin{gathered}
\Omega=2 \pi[1-\cos (\theta / 2)] \\
\theta=2 \arccos \left(1-\frac{2}{\mu}\right)
\end{gathered}
$$




\section{Supplementary Schemes and Figures}

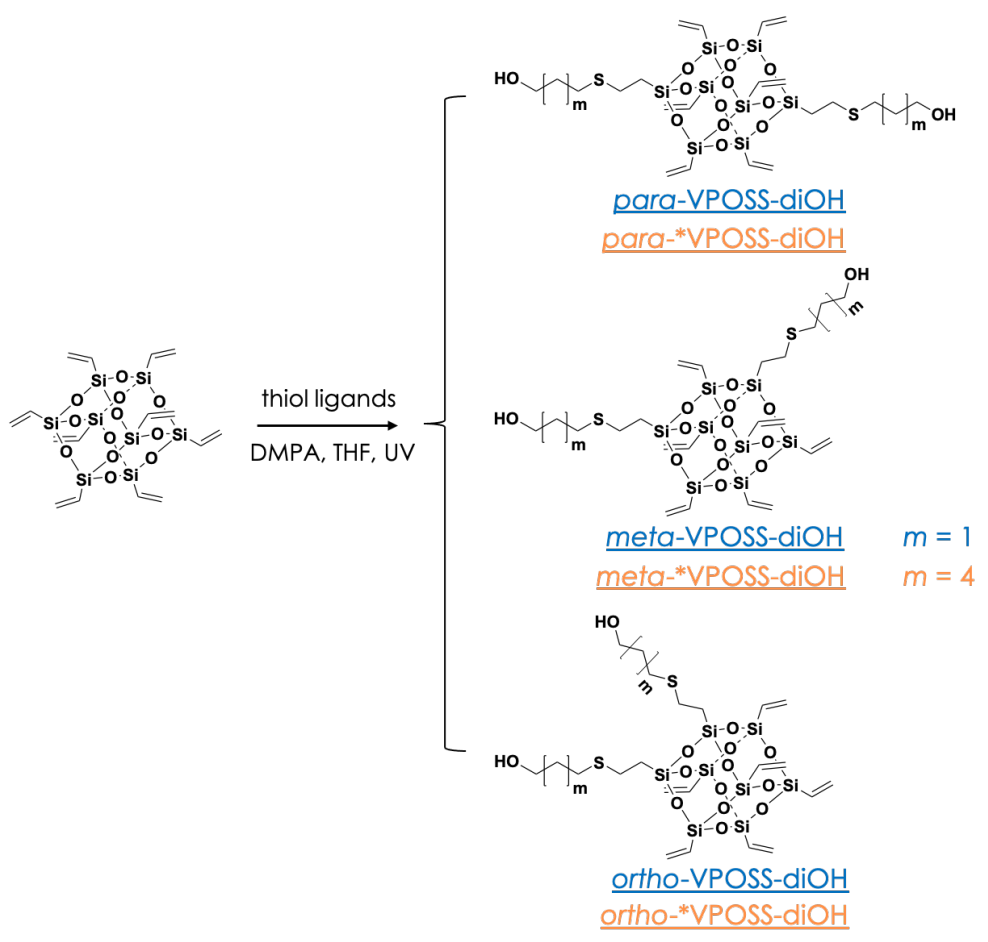

Scheme S1. Synthesis of octavinyl POSS with two hydroxyl substituting groups (VPOSS-diOH) at para-, meta-, and ortho-positions. Thiol ligands are 3-mercapto-1-propanol $(m=1)$ and 6-mercapto-1-hexanol $(m=$ 4).
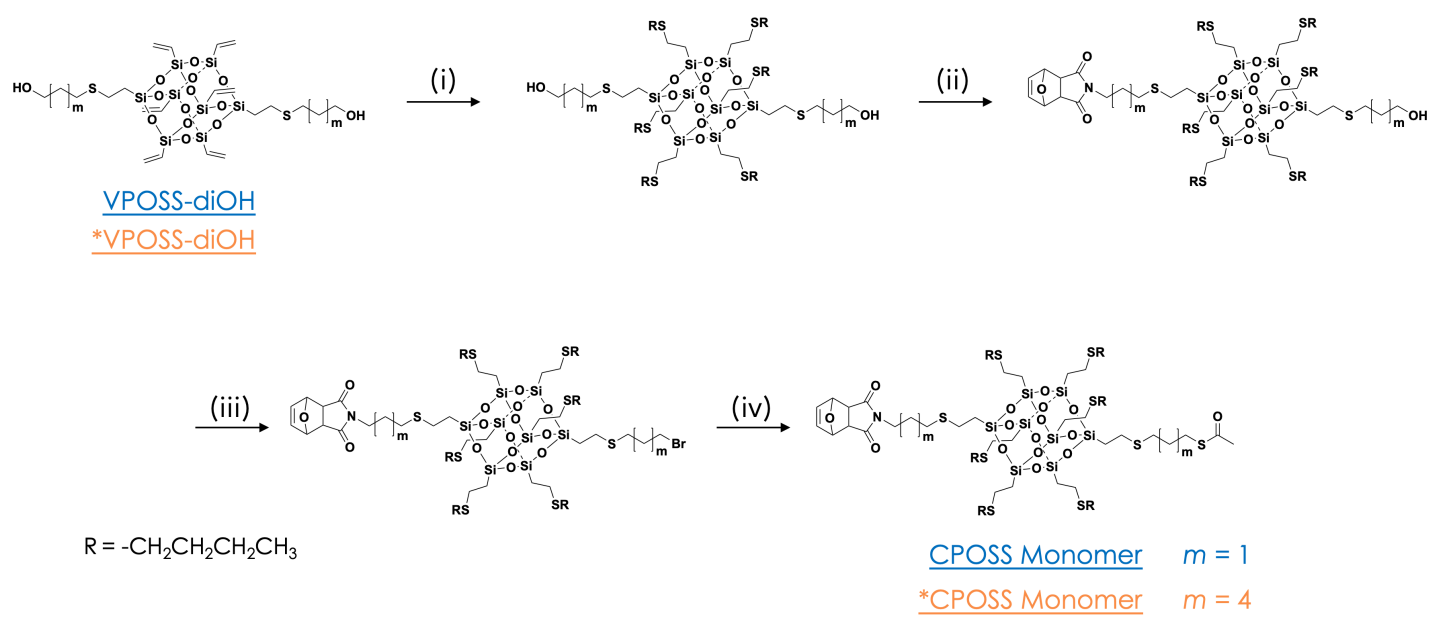

Scheme S2. Synthetic route of the CPOSS and *CPOSS monomer: (i) RSH, DMPA, THF, $25{ }^{\circ} \mathrm{C}, 365 \mathrm{~nm} \times$ 30 min; (ii) Furan protected maleimide, DIAD, $\mathrm{PPh}_{3}$, THF, $0{ }^{\circ} \mathrm{C}, 12 \mathrm{~h}$; (iii) $\mathrm{CBr}_{4}, \mathrm{PPh}_{3}, \mathrm{CH}_{2} \mathrm{Cl}_{2}, 0^{\circ} \mathrm{C}, 2 \mathrm{~h}$; (iv) potassium thioacetate, $\mathrm{KI}, \mathrm{THF} / \mathrm{MeOH}, 75^{\circ} \mathrm{C}, 12 \mathrm{~h}$. Take para-configuration as an example. 


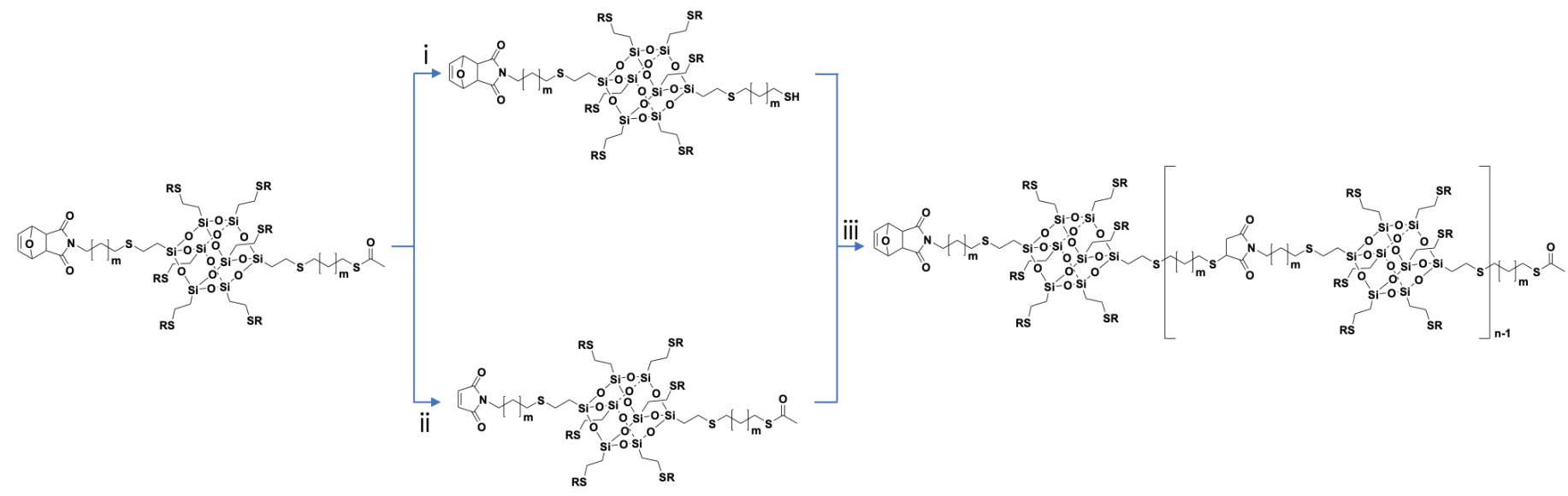

Scheme S3. Iterative exponential growth of discrete POSS chains: (i) acetyl chloride, $-78{ }^{\circ} \mathrm{C}, \mathrm{CH}_{2} \mathrm{Cl}_{2} / \mathrm{MeOH}$, $7 \mathrm{~h}$; (ii) toluene, $110^{\circ} \mathrm{C}, 5 \mathrm{~h}$; (iii) TEA, $\mathrm{CHCl}_{3}, 25^{\circ} \mathrm{C}, 8 \mathrm{~h}$. Take para-configuration as an example.

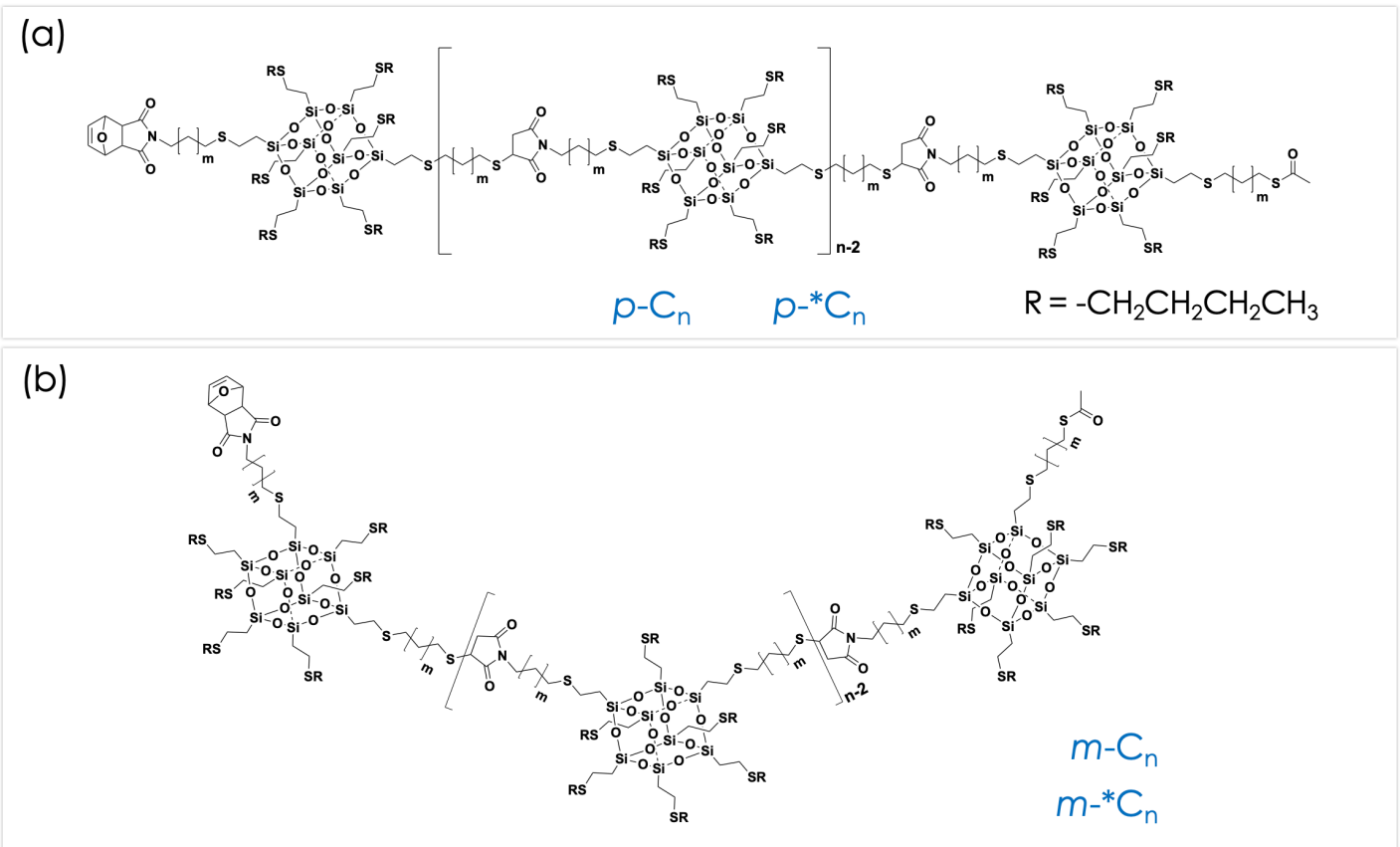

(c)

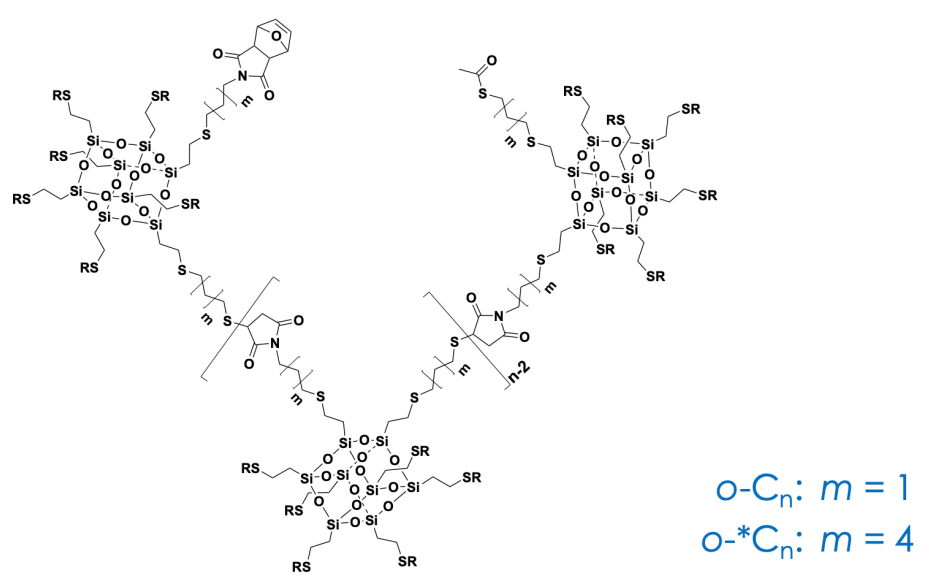

Scheme S4. Giant polymeric chains $\mathrm{C}_{n}$ with para- (a), meta- (b), and ortho-configuration (c). 


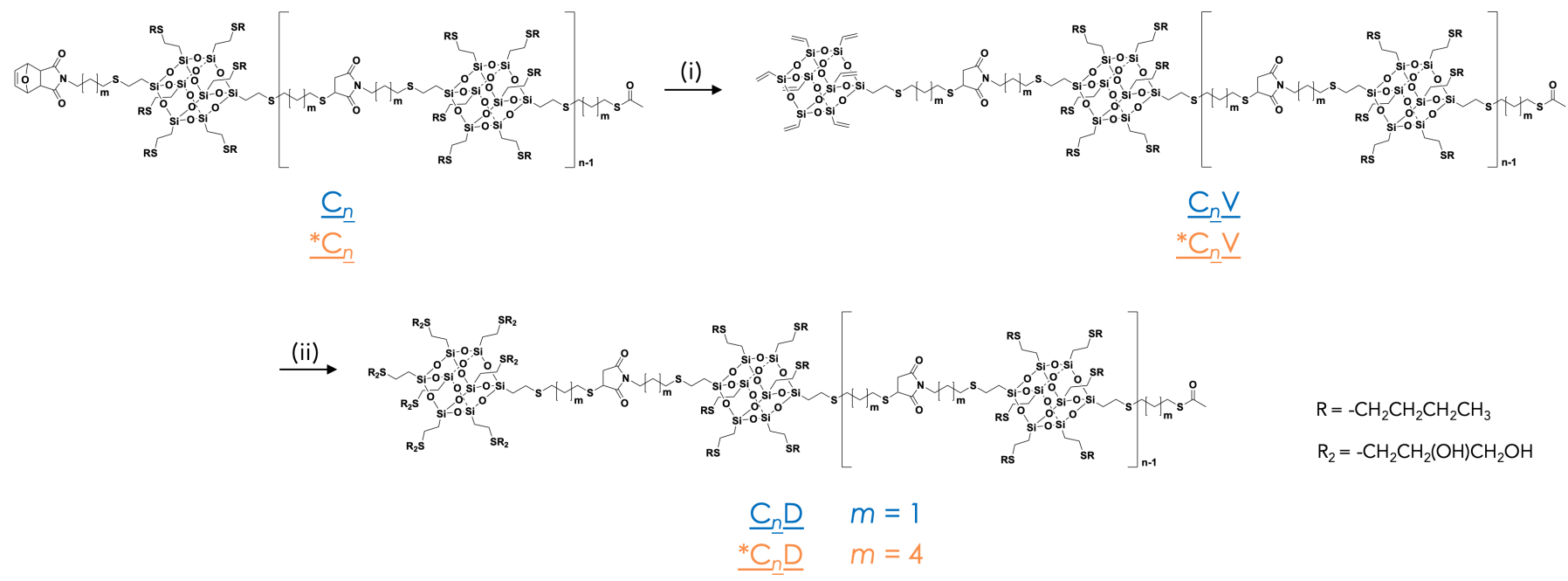

Scheme S5. The general synthetic strategy for amphiphilic giant polymeric chain $p-\mathrm{C}_{n} \mathrm{D}$ : (i) Toluene, $110{ }^{\circ} \mathrm{C}$, $5 \mathrm{~h}$; (ii) V7POSS-SH, TEA, $\mathrm{CHCl}_{3}, 25^{\circ} \mathrm{C}, 8 \mathrm{~h}$; (iii) 1-thioglycerol, DMPA, THF, $25^{\circ} \mathrm{C}, 365 \mathrm{~nm} \times 35 \mathrm{~min}$. Take para-configuration as an example. 
O-VPOSS-diOH
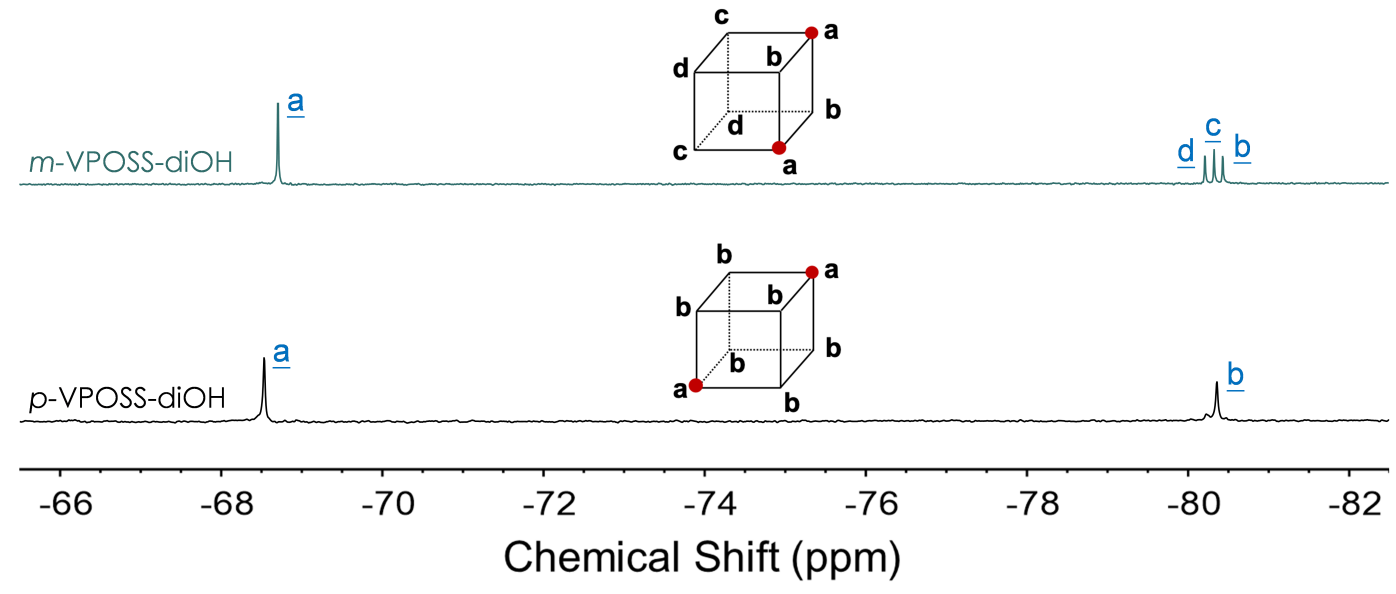

Figure S1. ${ }^{29} \mathrm{Si}$ NMR spectra of VPOSS-diOH with para-, meta-, and ortho-configuration.
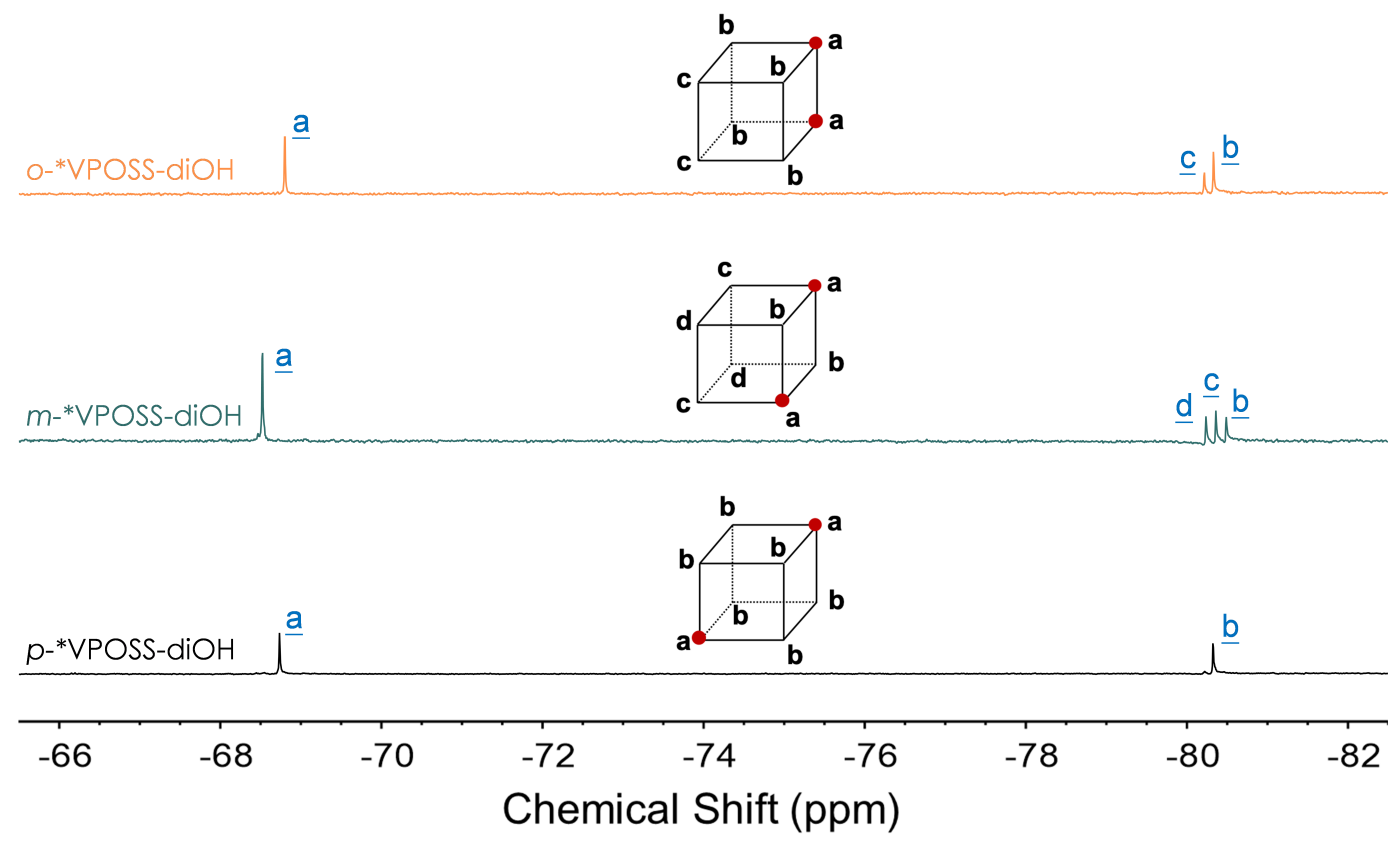

Figure S2. ${ }^{29} \mathrm{Si}$ NMR spectra of *VPOSS-diOH with para-, meta-, and ortho-configuration. 


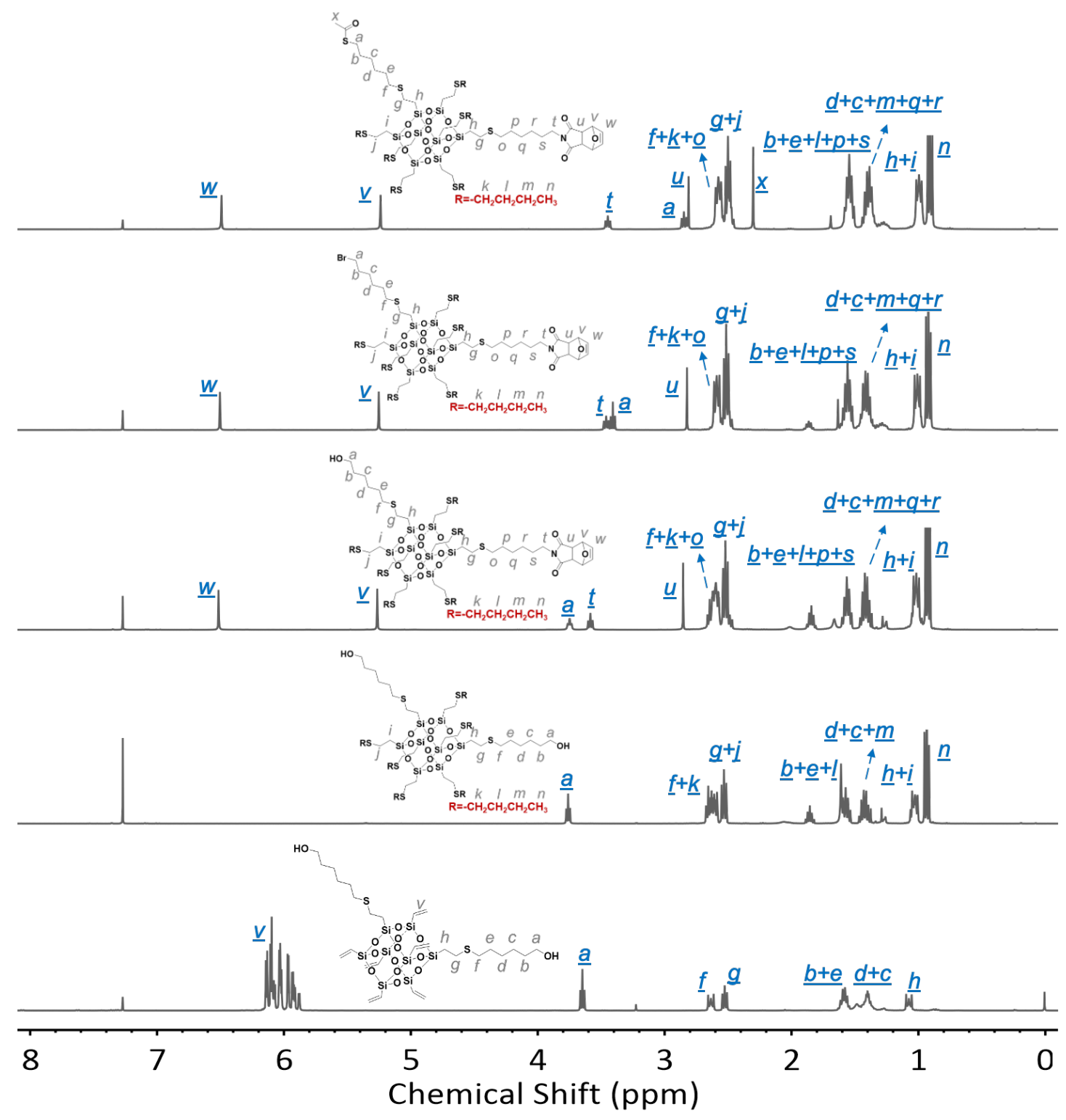

Figure S3. ${ }^{1} \mathrm{H}$ NMR spectra of * CPOSS monomer and related intermediate compounds. Take meta-configuration as an example. 


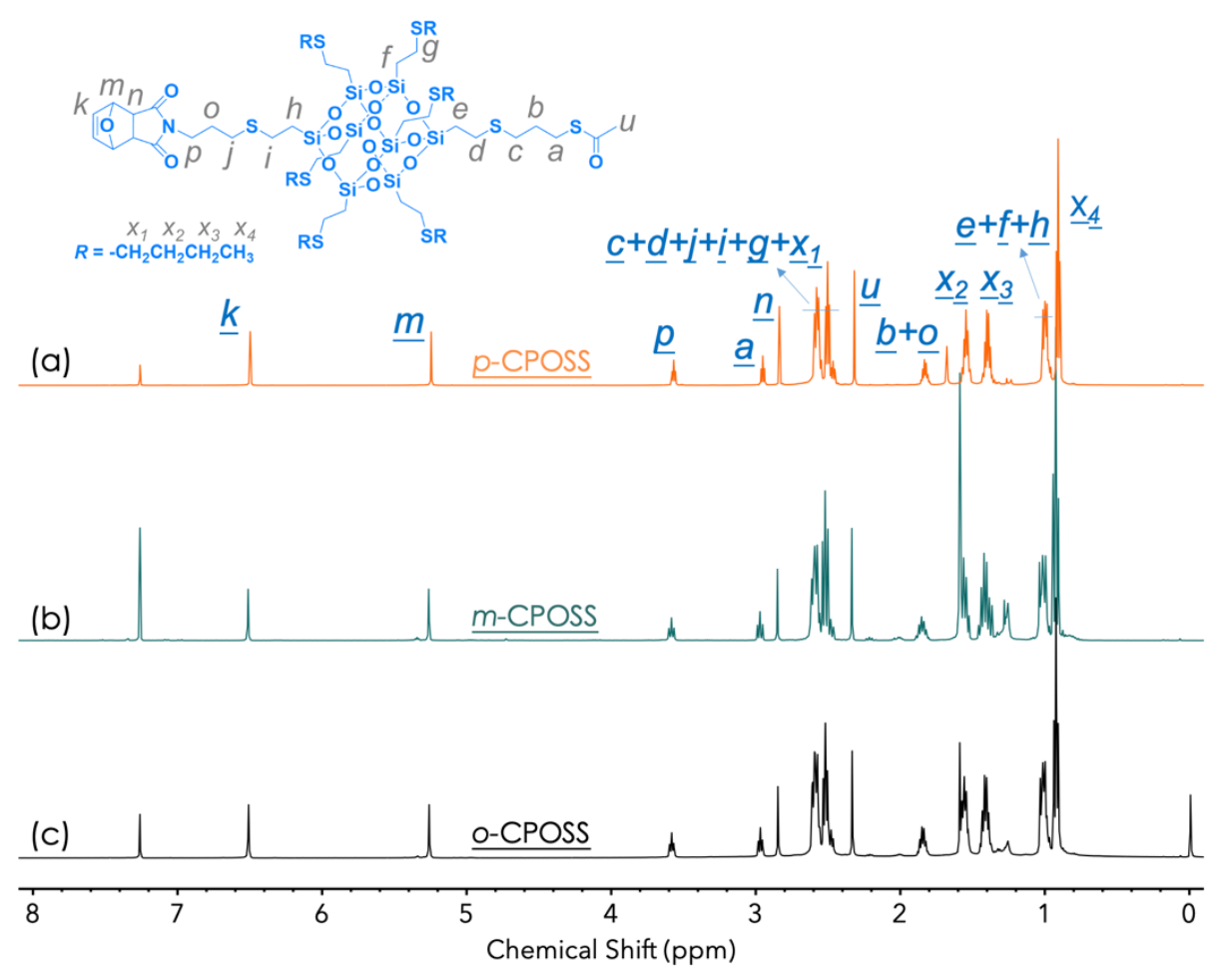

Figure S4. ${ }^{1} \mathrm{H}$ NMR spectra of CPOSS monomer with para- (a), meta- (b), and ortho-configuration (c).

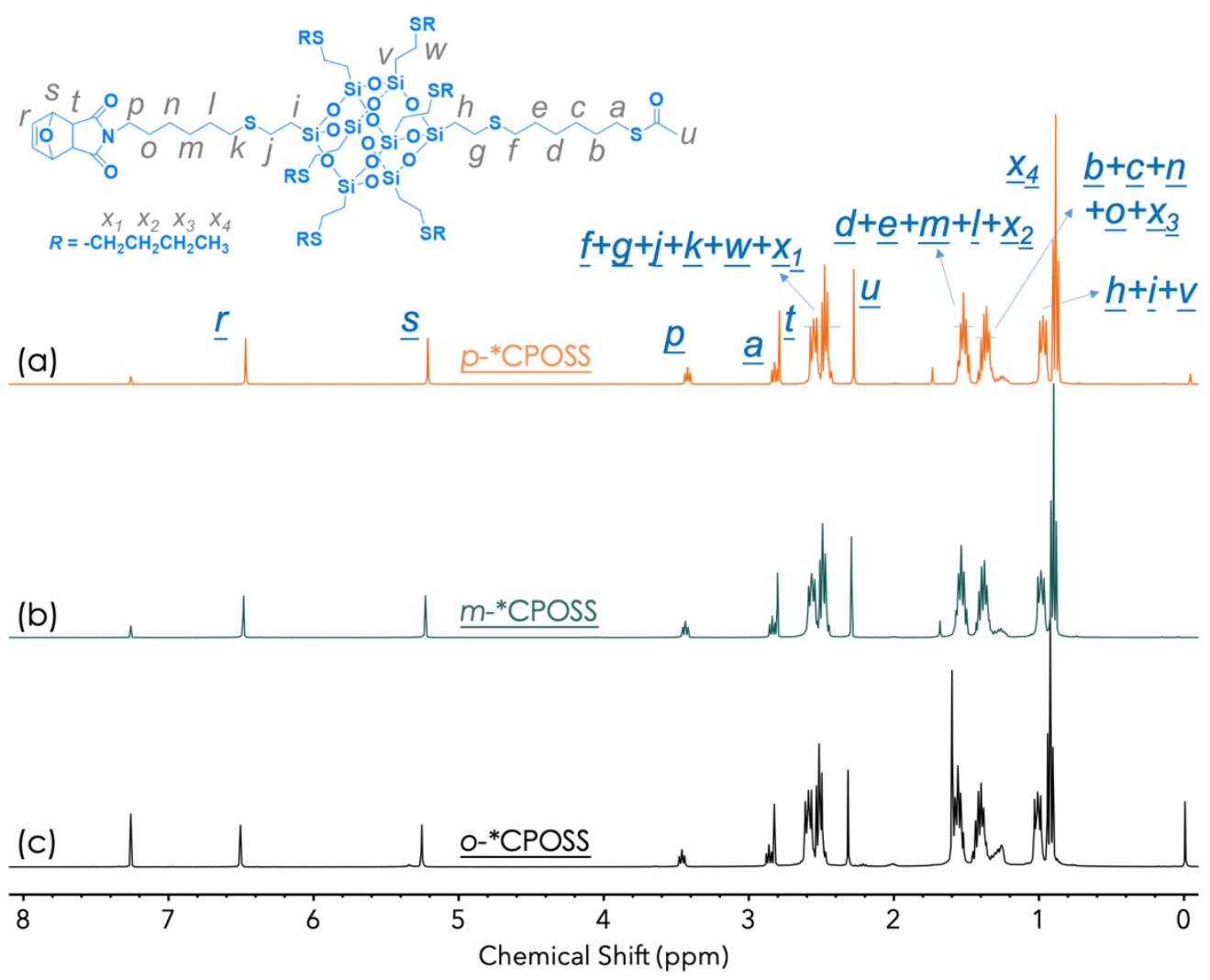

Figure S5. ${ }^{1} \mathrm{H}$ NMR spectra of *CPOSS monomer with para- (a), meta- (b), and ortho-configuration (c). 
(a)
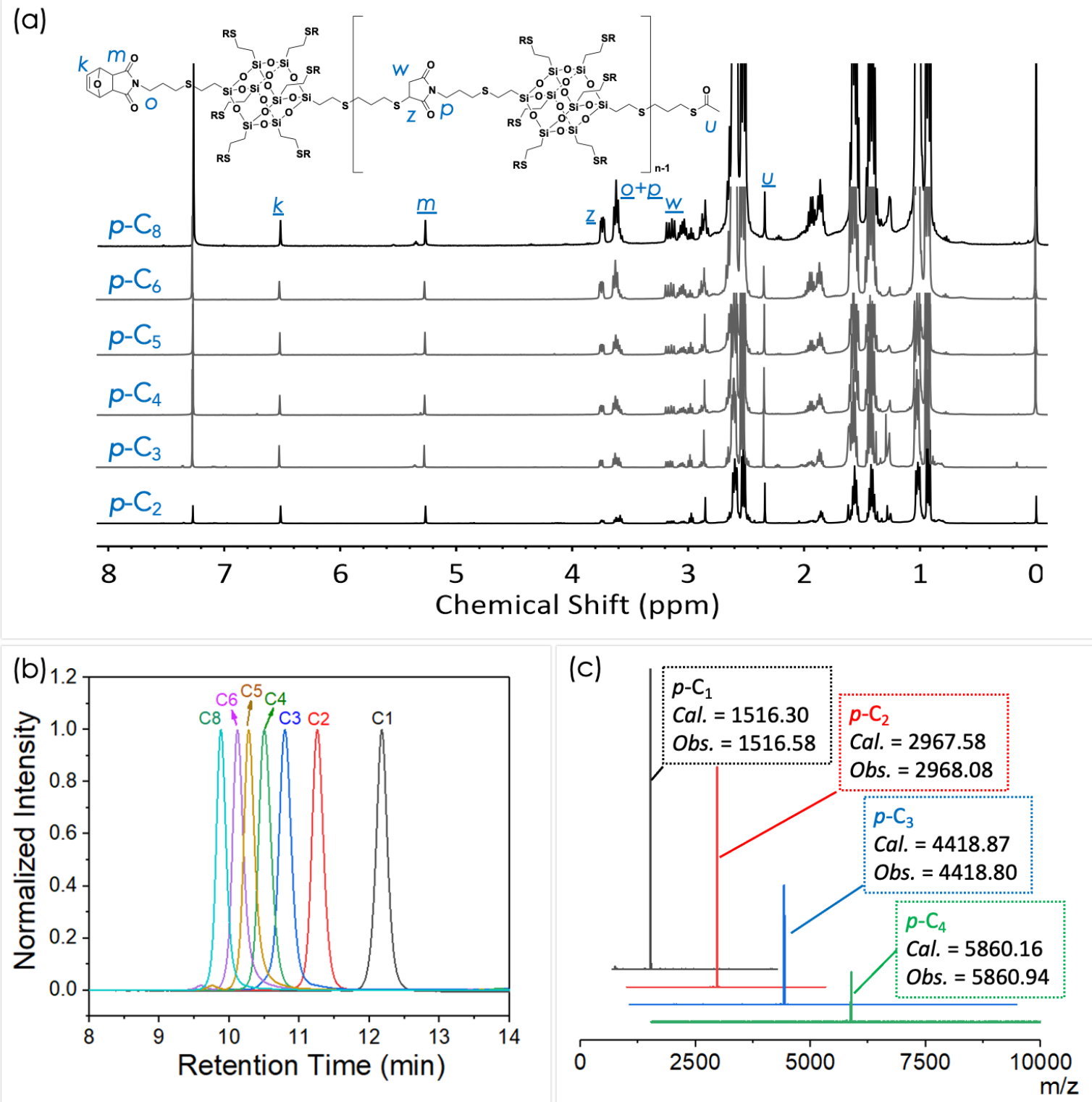

Figure S6. ${ }^{1} \mathrm{H}$ NMR spectra (a), SEC profiles (b), and MALDI-ToF MS (c) of $p$ - $\mathrm{C}_{n}$ chains. $m$ - and $o-\mathrm{C}_{n}$ have similar spectra (data not shown). 


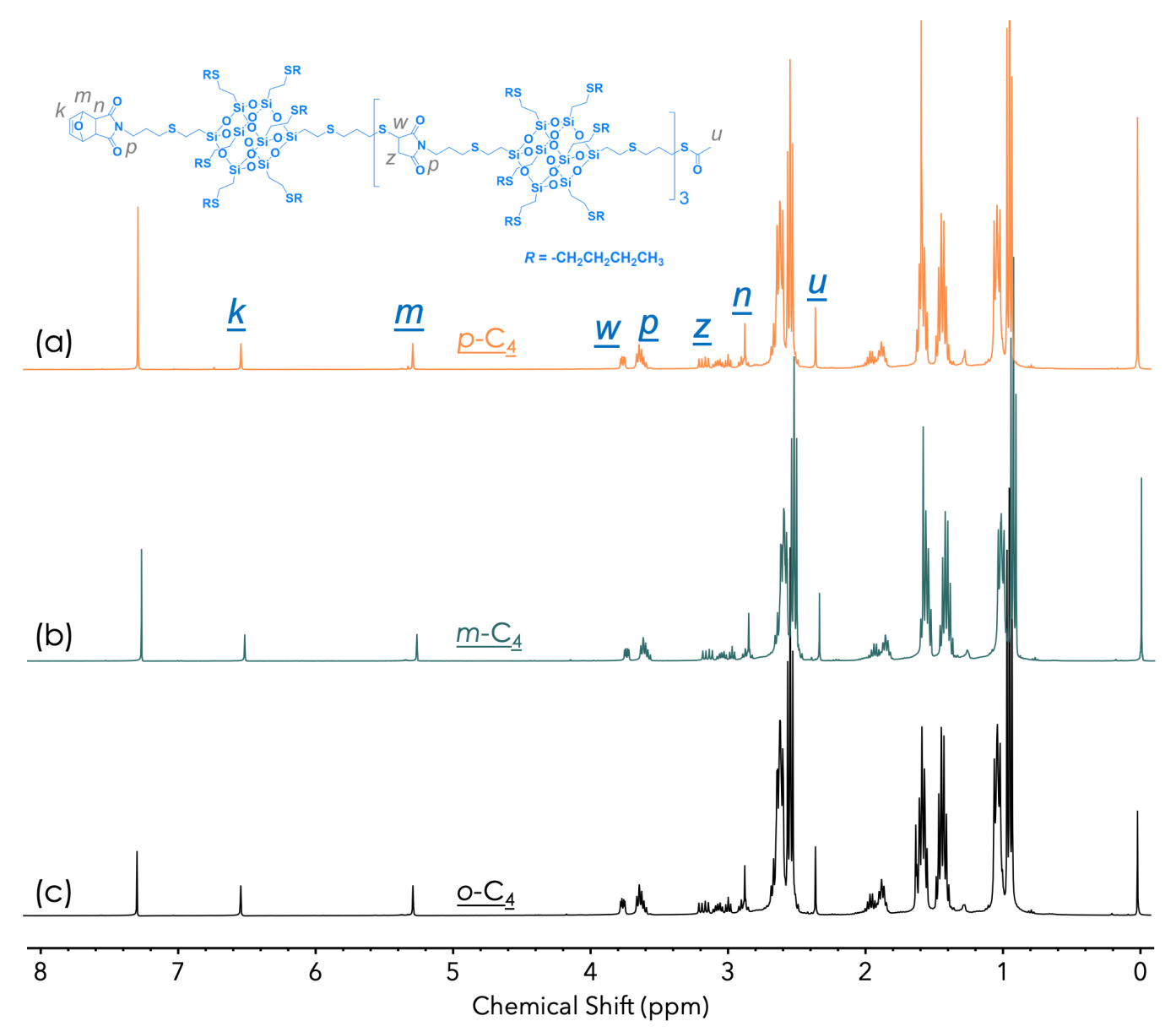

Figure S7. ${ }^{1} \mathrm{H}$ NMR spectra of $\mathrm{C}_{4}$ chains with para- (a), meta- (b), and ortho-configuration (c).

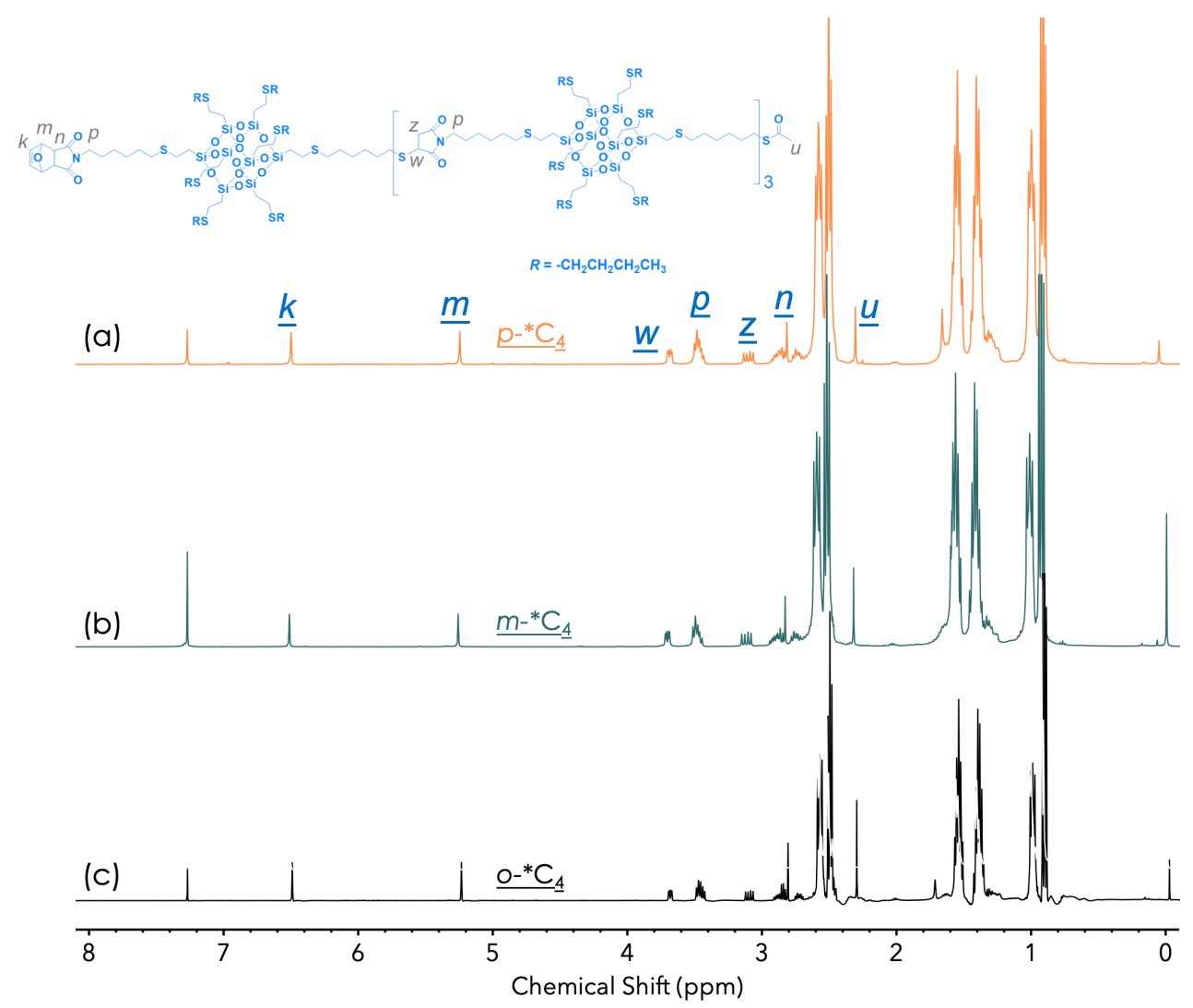

Figure S8. ${ }^{1} \mathrm{H}$ NMR spectra of $* \mathrm{C}_{4}$ chains with para- (a), meta- (b), and ortho-configuration (c). 

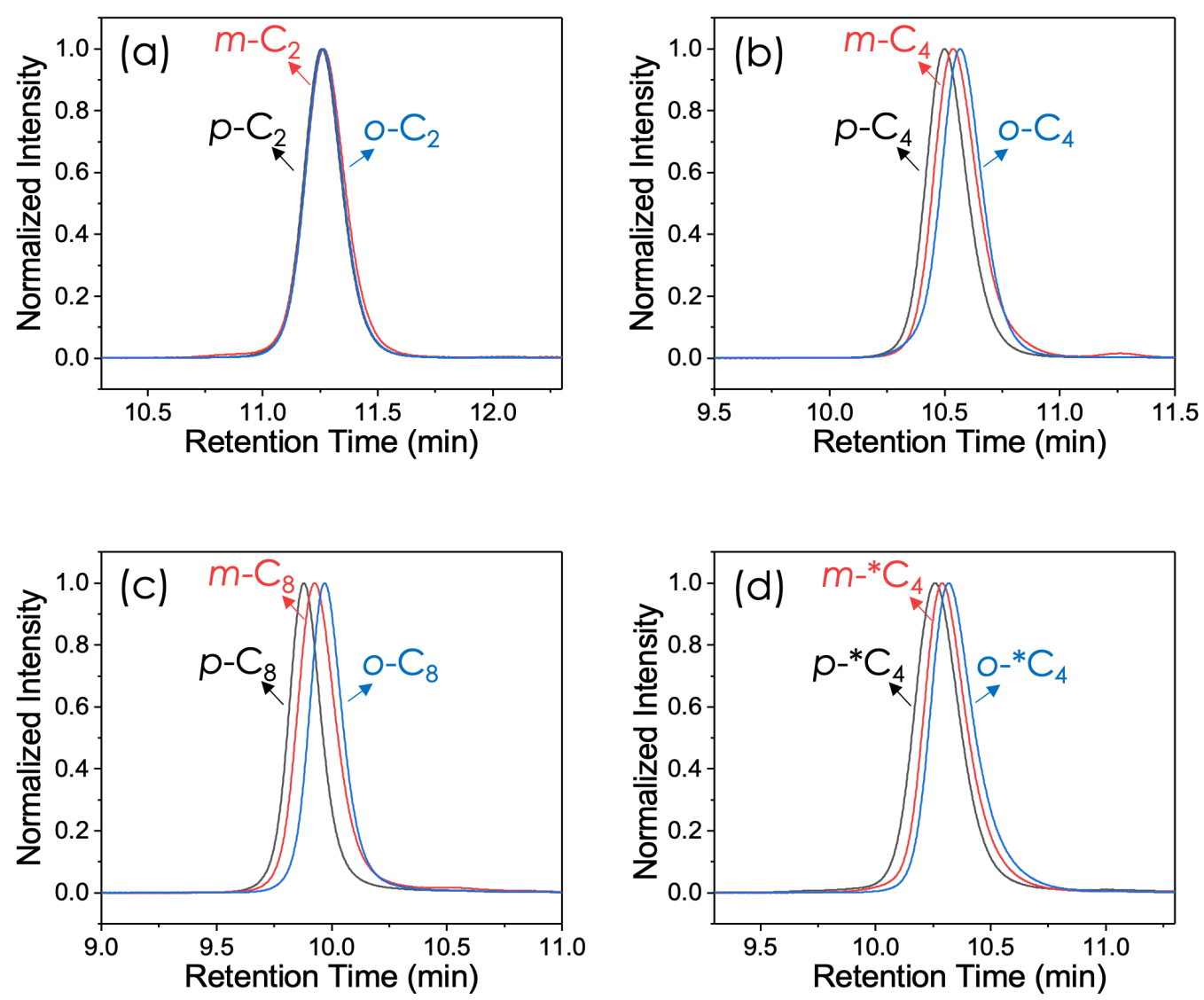

Figure S9. SEC profiles of the regioisomers with different $n$. 

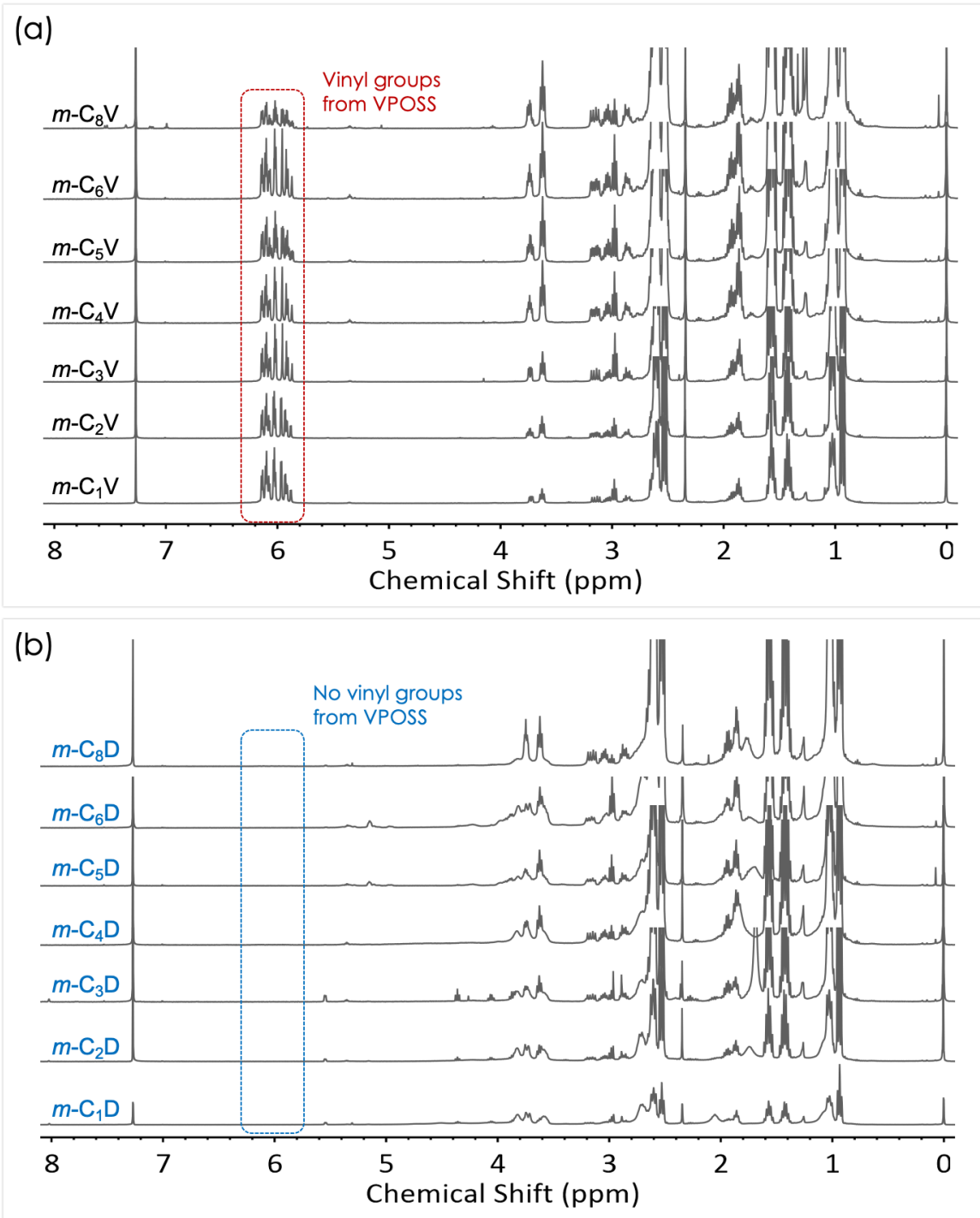

Figure S10. ${ }^{1} \mathrm{H}$ NMR spectra and SEC profiles of meta $-\mathrm{C}_{n} \mathrm{~V}(\mathrm{a})$ and meta $-\mathrm{C}_{n} \mathrm{D}(\mathrm{b})$, respectively.
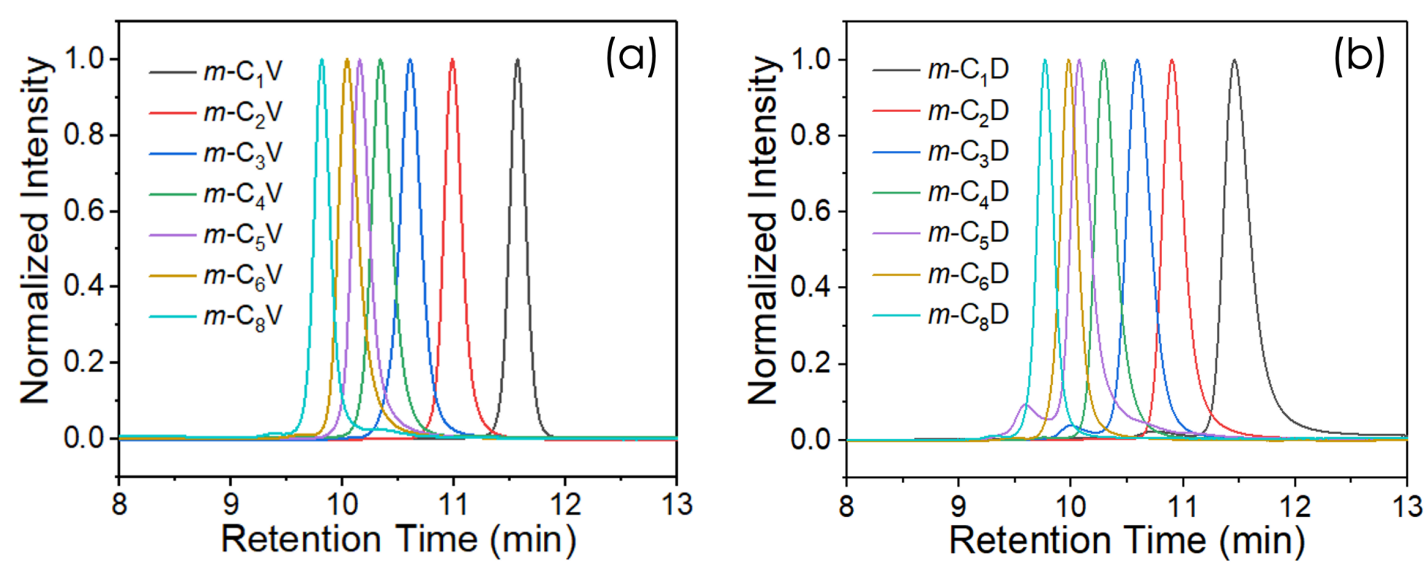

Figure S11. ${ }^{1} \mathrm{H}$ NMR spectra and SEC profiles of meta- $\mathrm{C}_{n} \mathrm{~V}(\mathrm{a})$ and meta- $\mathrm{C}_{n} \mathrm{D}(\mathrm{b})$, respectively. 
(a)
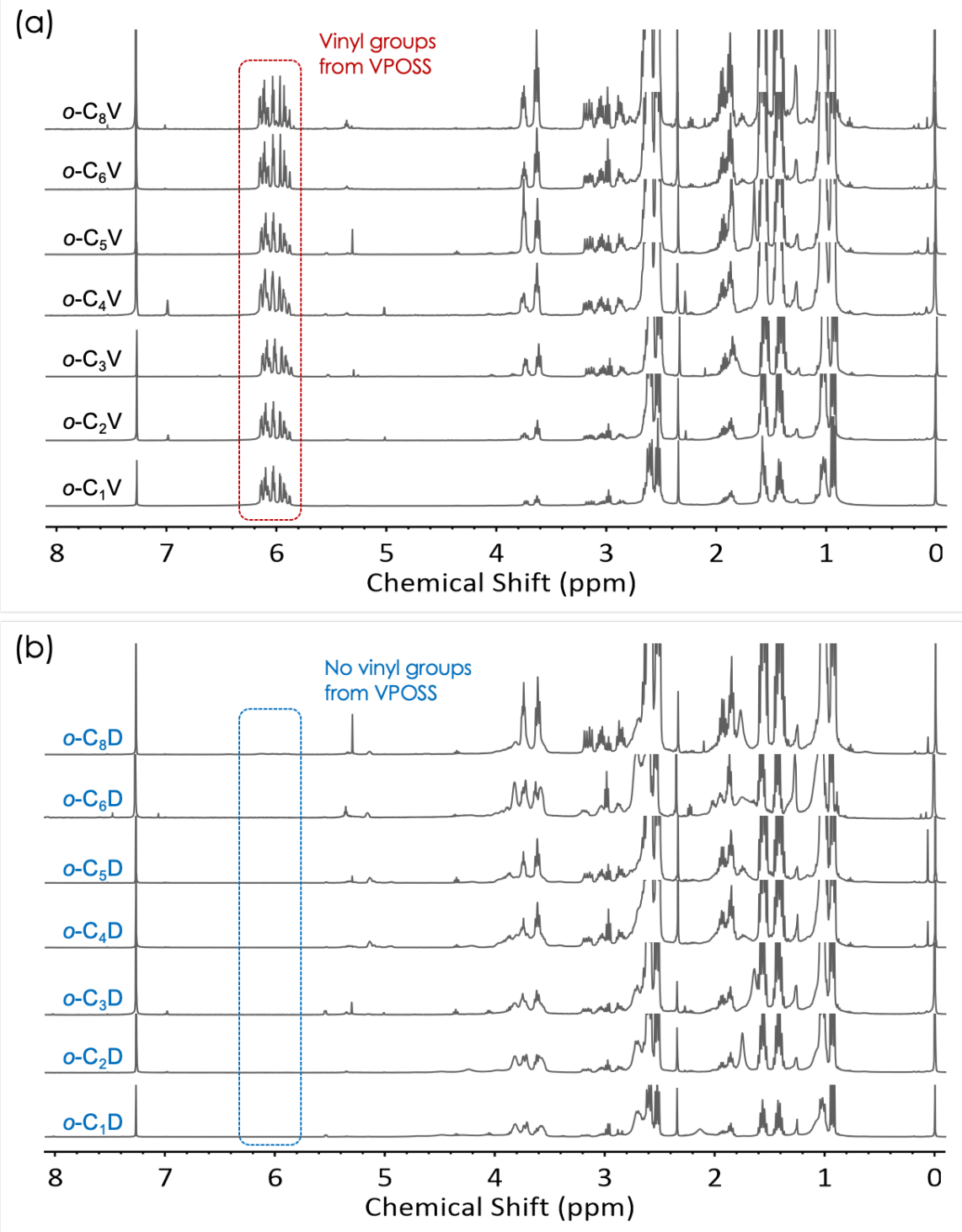

Figure S12. ${ }^{1} \mathrm{H}$ NMR spectra and SEC profiles of ortho- $\mathrm{C}_{n} \mathrm{~V}(\mathrm{a})$ and ortho- $\mathrm{C}_{n} \mathrm{D}(\mathrm{b})$, respectively.
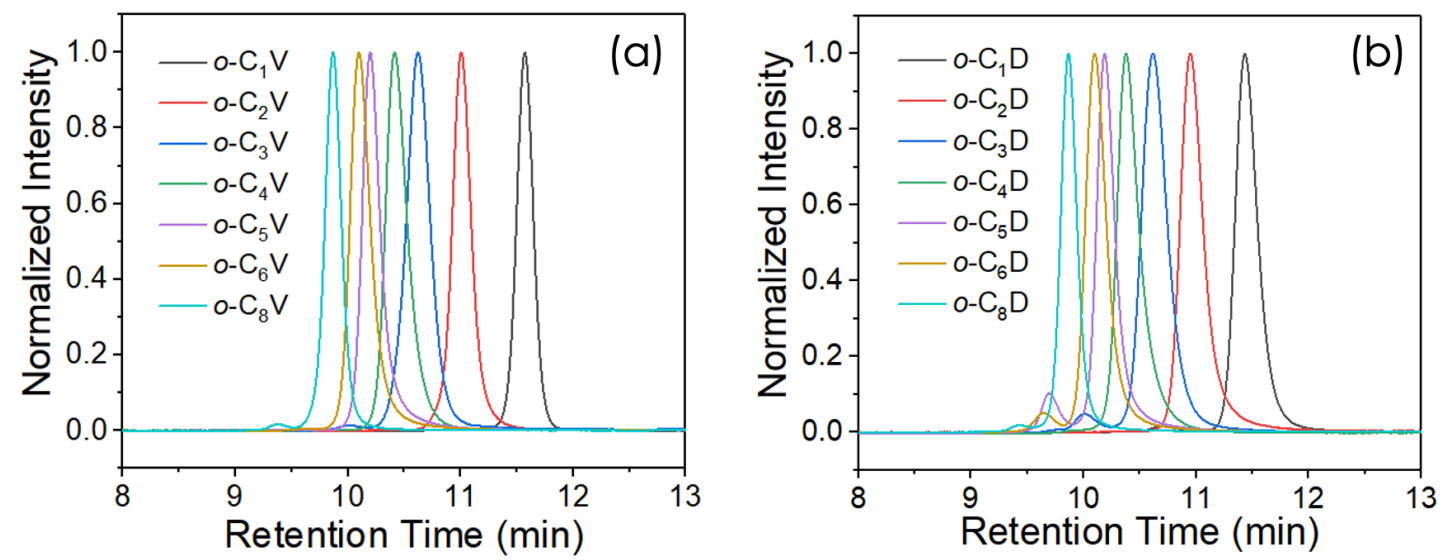

Figure S13. ${ }^{1} \mathrm{H}$ NMR spectra and SEC profiles of ortho- $\mathrm{C}_{n} \mathrm{~V}(\mathrm{a})$ and ortho- $\mathrm{C}_{n} \mathrm{D}(\mathrm{b})$, respectively. 


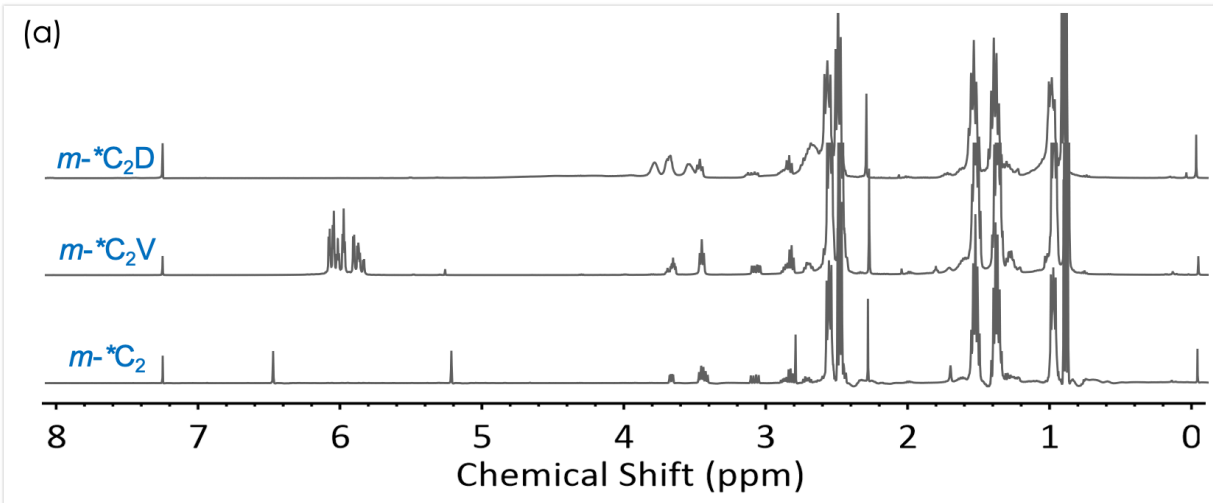

(c)

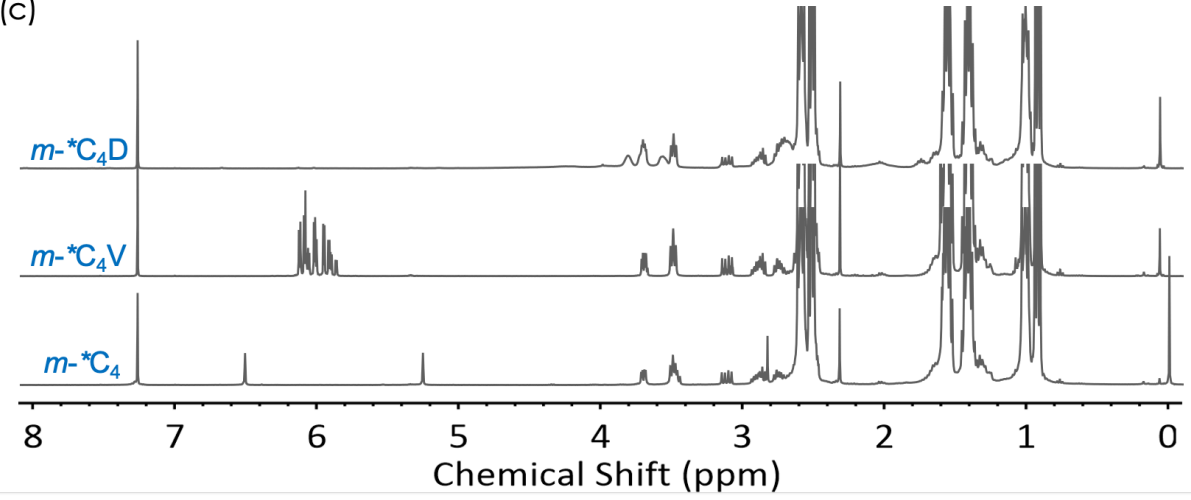

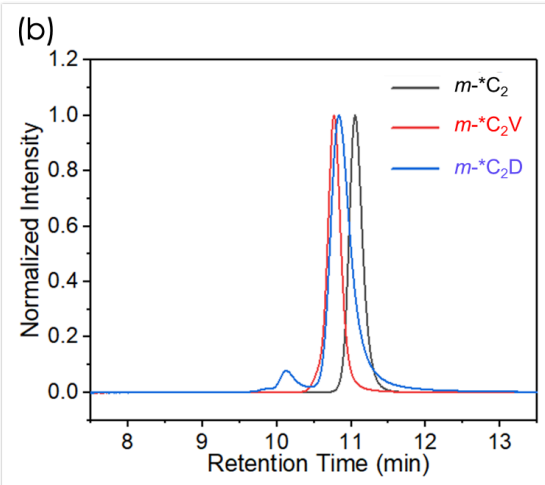

(d)

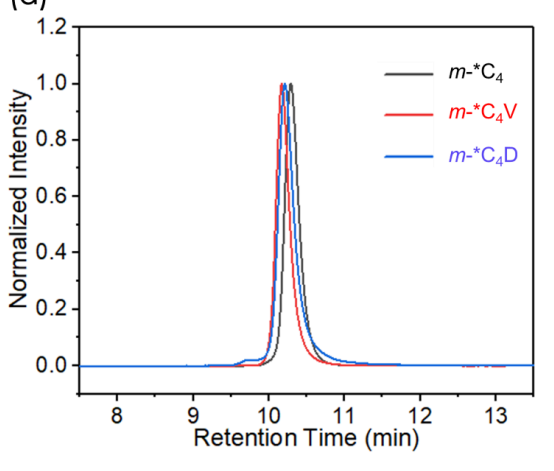

Figure S14. ${ }^{1} \mathrm{H}$ NMR spectra (a, c), and SEC profiles (b, d) of $m-{ }^{*} \mathrm{C}_{n} \mathrm{D}(n=2,4)$.

(a)

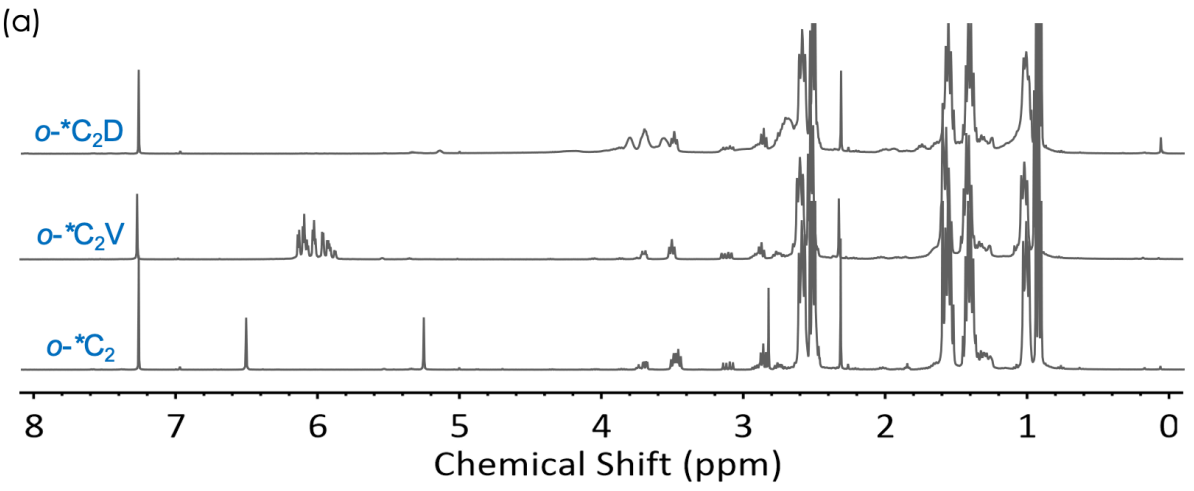

(c)

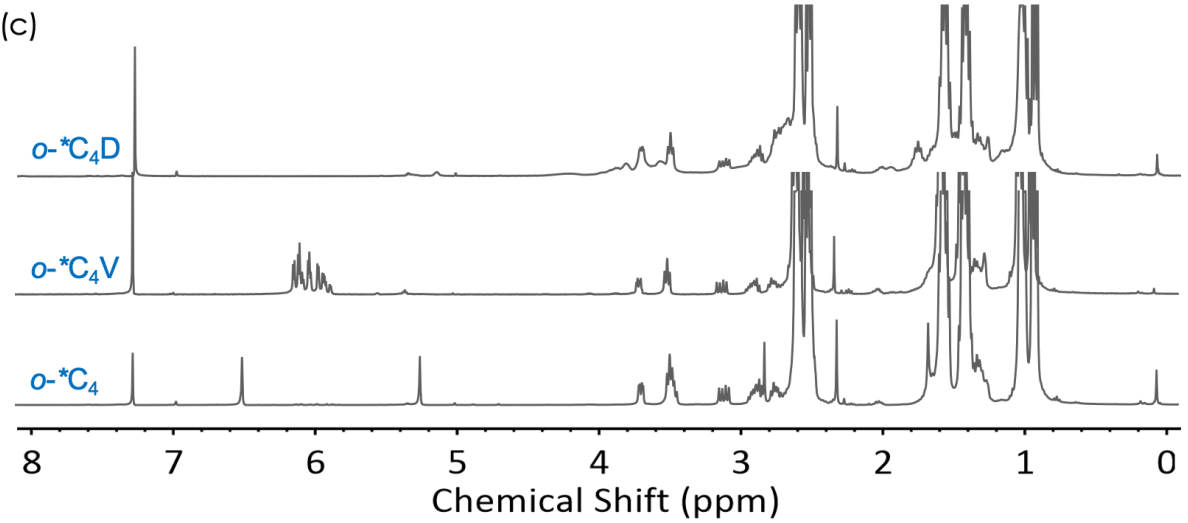

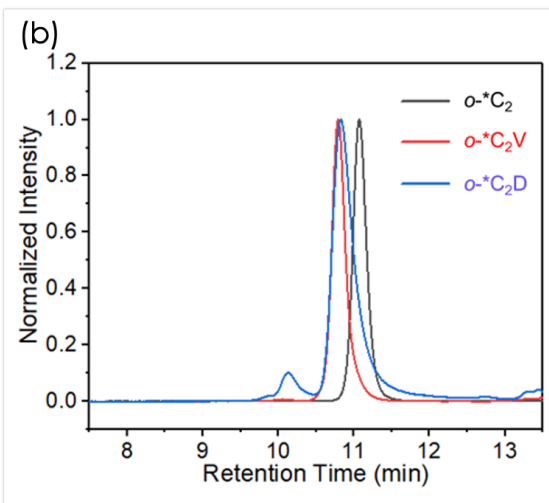

(d)

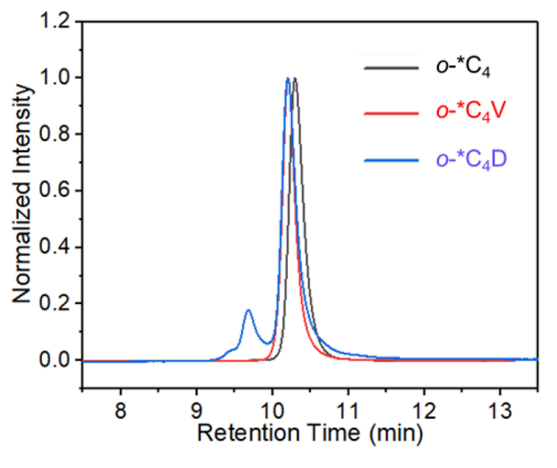

Figure S15. ${ }^{1} \mathrm{H}$ NMR spectra (a, c), and SEC profiles (b, d) of $o-{ }^{*} \mathrm{C}_{n} \mathrm{D}(n=2,4)$. 

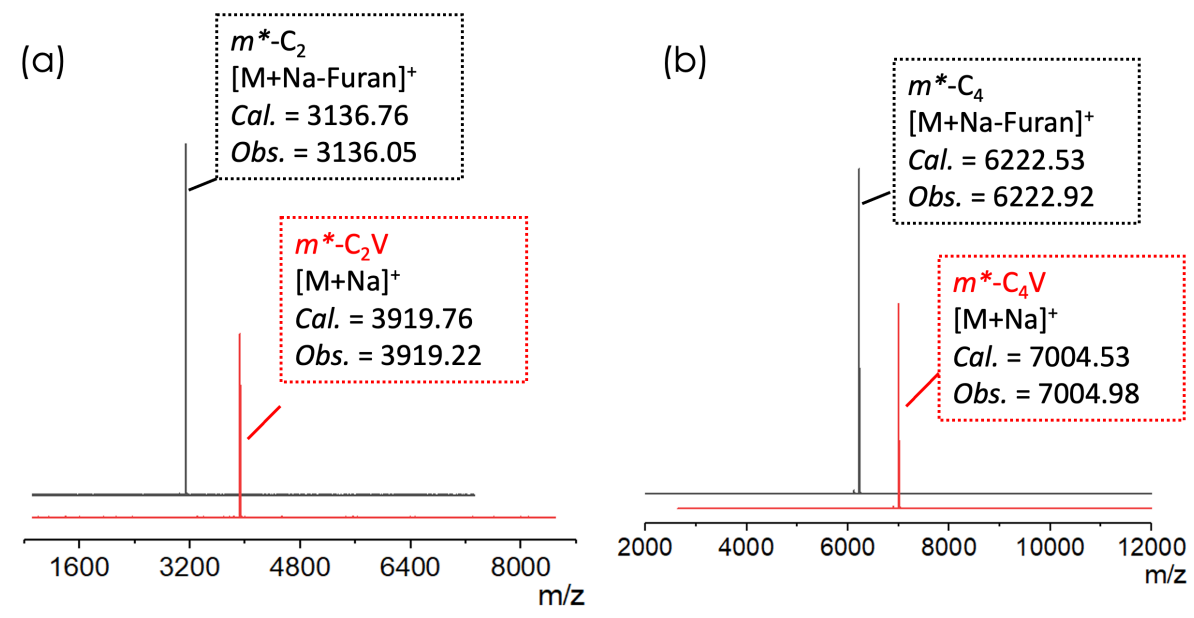

Figure S16. MALDI-ToF MS of $m^{*}{ }_{-} \mathrm{C}_{n} \mathrm{D}$.
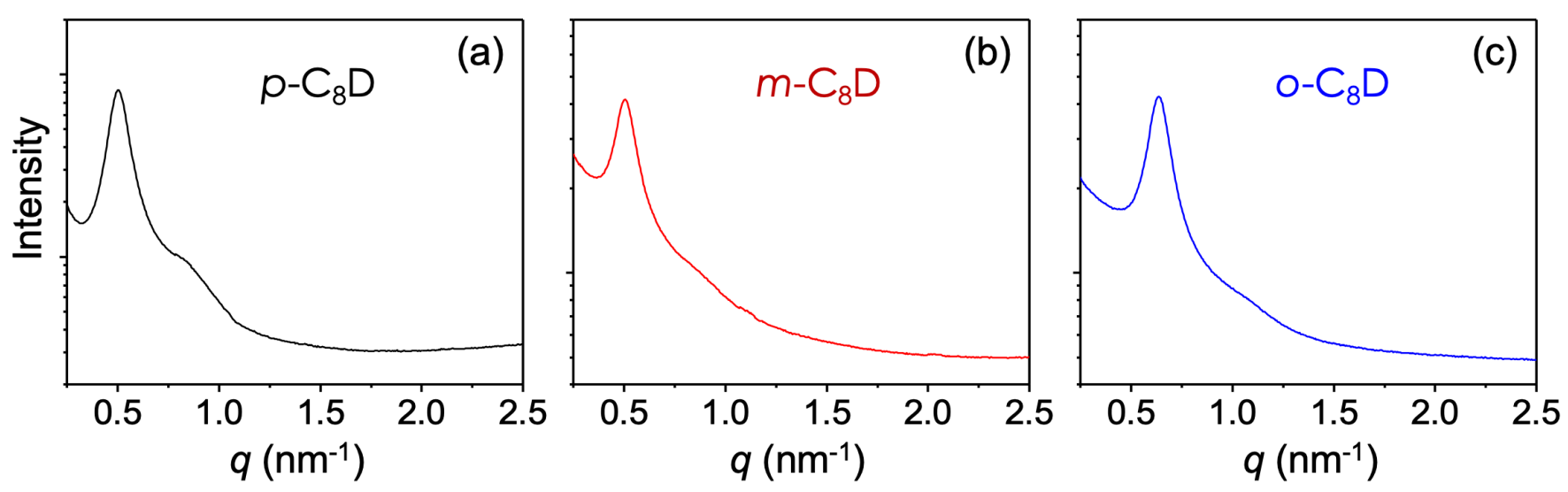

Figure S17. SAXS patterns of $p-\mathrm{C}_{8} \mathrm{D}(\mathrm{a}), m-\mathrm{C}_{8} \mathrm{D}(\mathrm{b})$, and $o-\mathrm{C}_{8} \mathrm{D}(\mathrm{c})$.

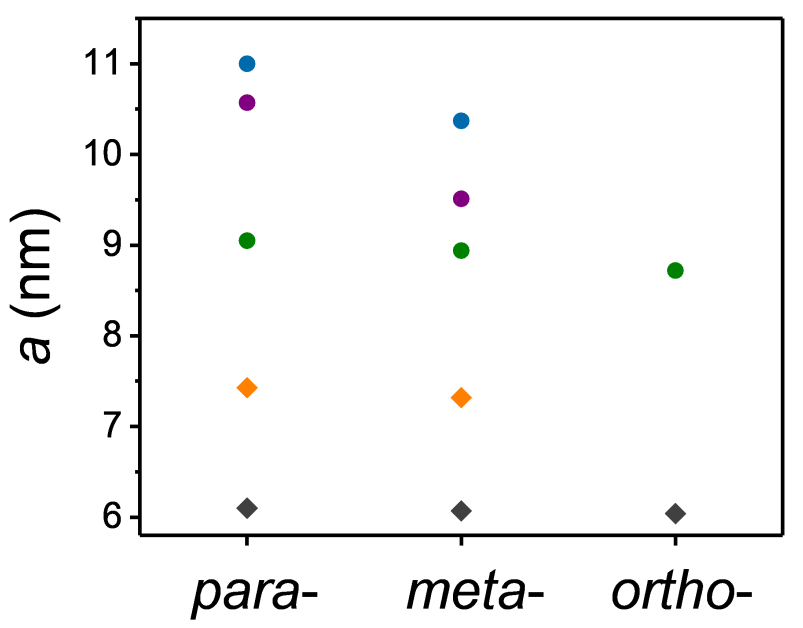

Figure S18. Difference of the lattice dimension between regioisomers with the same phase structures: lamellar periodicities for $n=1$ and 2 (square), and inter-column distances of HEX for $n=3,4$, and 5 (circle). Data adopted from Table 1. 


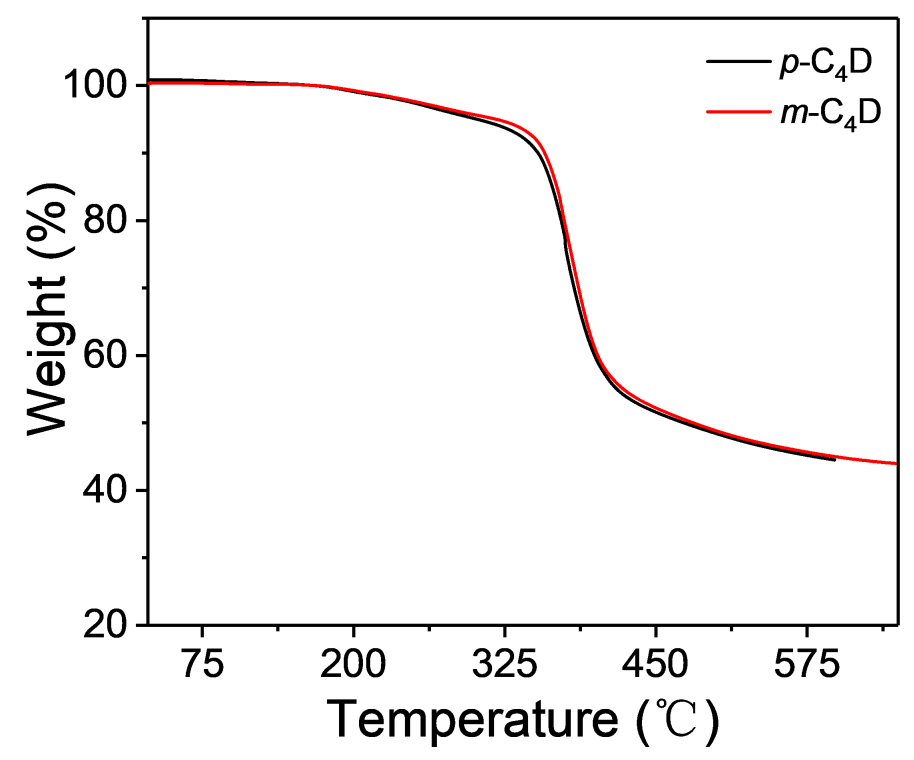

Figure S19. TGA curves of $p-\mathrm{C}_{4} \mathrm{D}$ and $m-\mathrm{C}_{4} \mathrm{D}$. Heating rate: $10{ }^{\circ} \mathrm{C} / \mathrm{min}$.

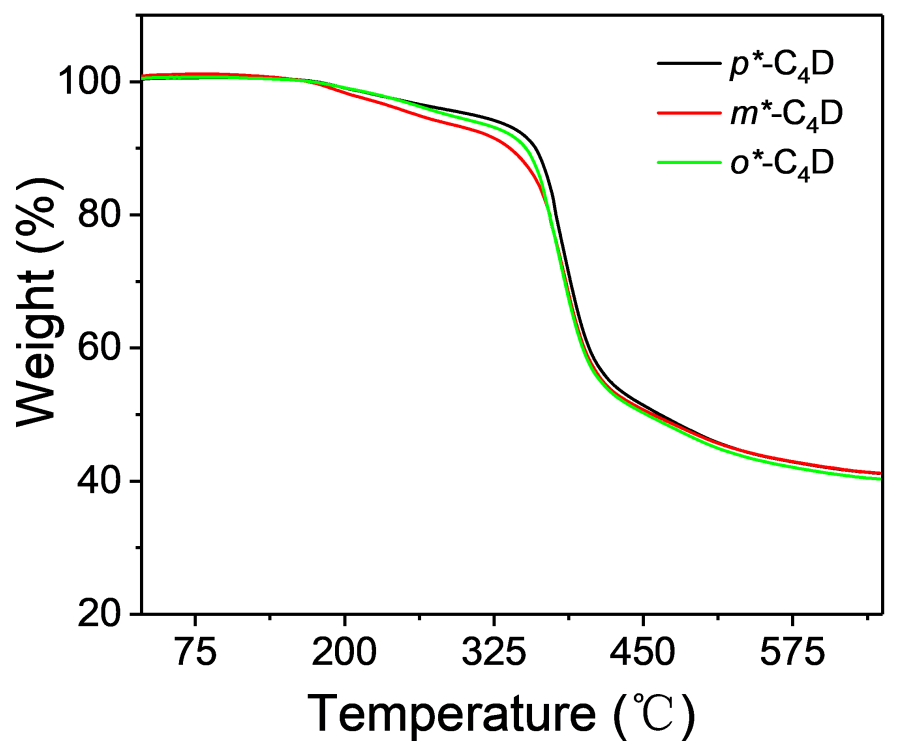

Figure S20. TGA curves of $p^{*} \mathrm{C}_{4} \mathrm{D}, m^{*}-\mathrm{C}_{4} \mathrm{D}$, and $o^{*}{ }_{-} \mathrm{C}_{4} \mathrm{D}$. Heating rate: $10{ }^{\circ} \mathrm{C} / \mathrm{min}$. 

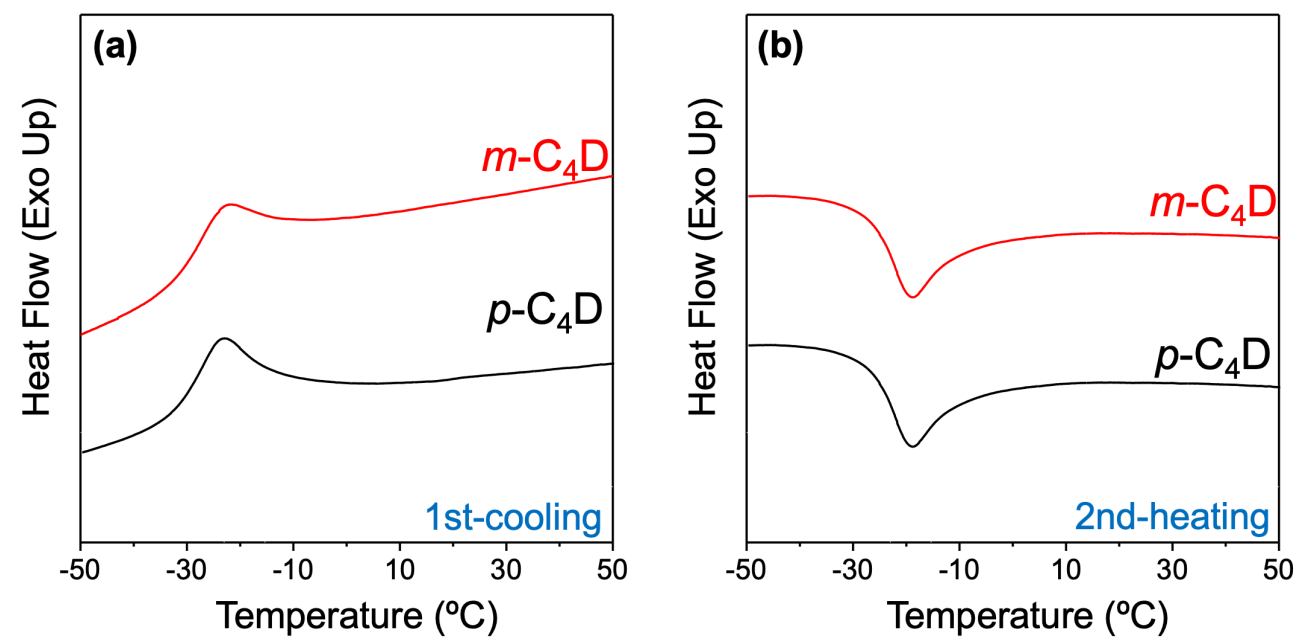

Figure S21. DSC thermograms of the $p-\mathrm{C}_{4} \mathrm{D}$ in the cooling (a) and heating (b) cycle. Heating/cooling rate: $5{ }^{\circ} \mathrm{C} / \mathrm{min}$.
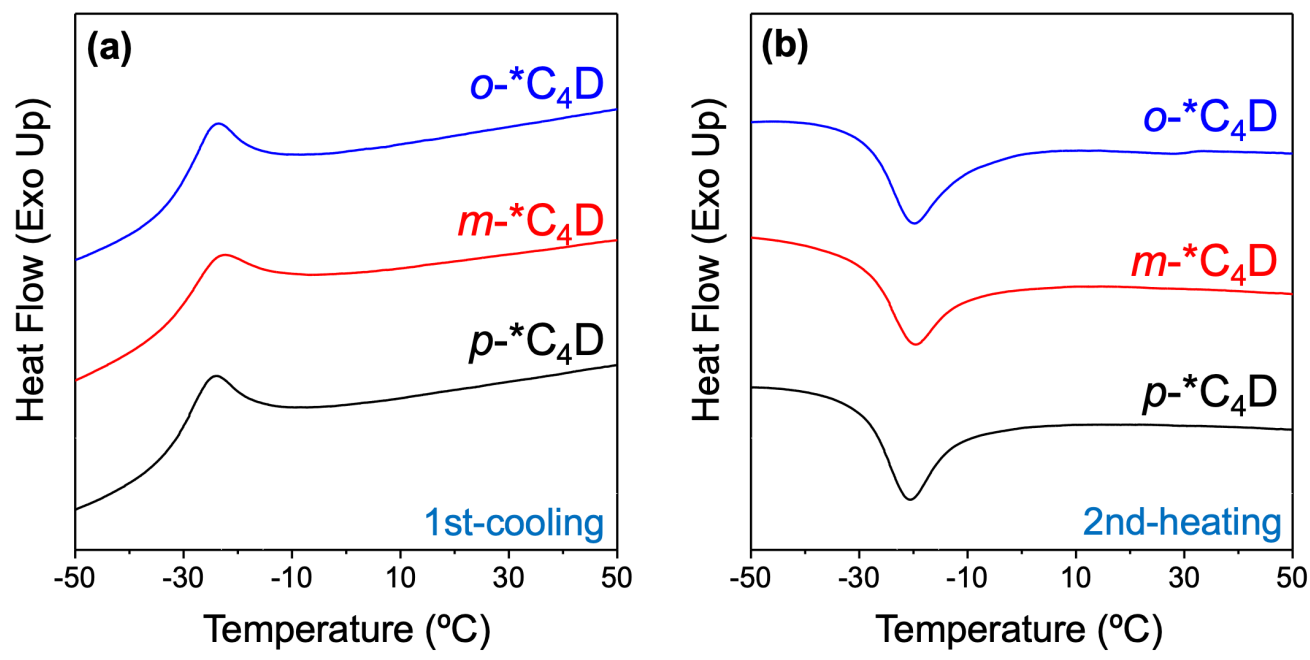

Figure S22. DSC thermograms of the $p^{*} \mathrm{C}_{4} \mathrm{D}, m^{*}-\mathrm{C}_{4} \mathrm{D}$, and $o^{*} \mathrm{C}_{4} \mathrm{D}$ in the cooling (a) and heating (b) cycle. Heating/cooling rate: $5^{\circ} \mathrm{C} / \mathrm{min}$.
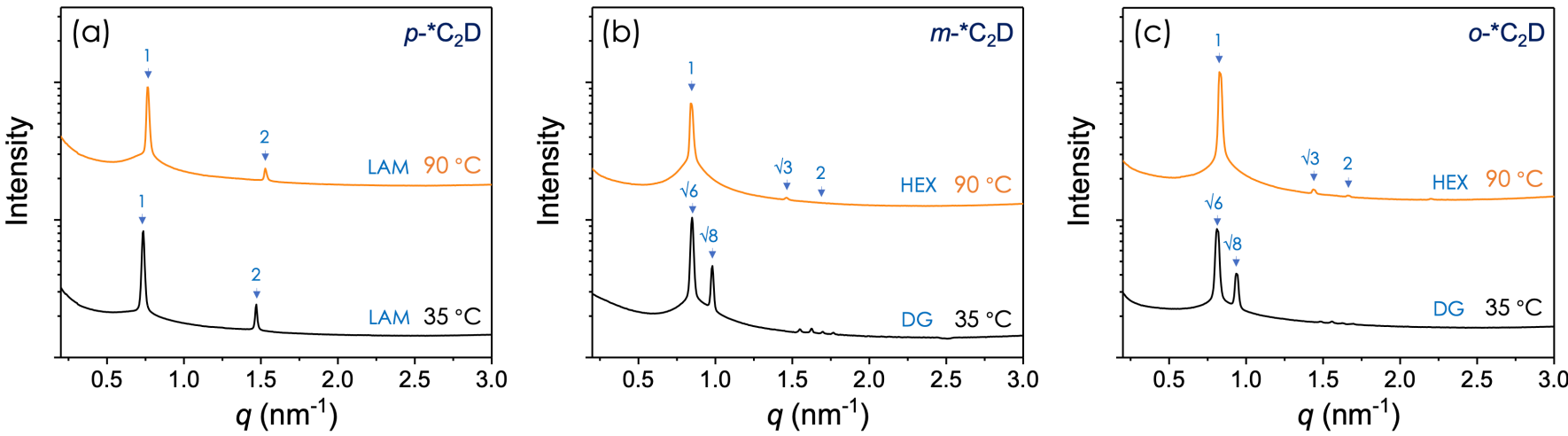

Figure S23. Temperature dependent SAXS profiles of the amphiphilic POSS chains: $p-{ }^{*} \mathrm{C}_{2} \mathrm{D}(\mathrm{a}), m-{ }^{*} \mathrm{C}_{2} \mathrm{D}$ (b), and $o-* \mathrm{C}_{2} \mathrm{D}(\mathrm{c})$. 

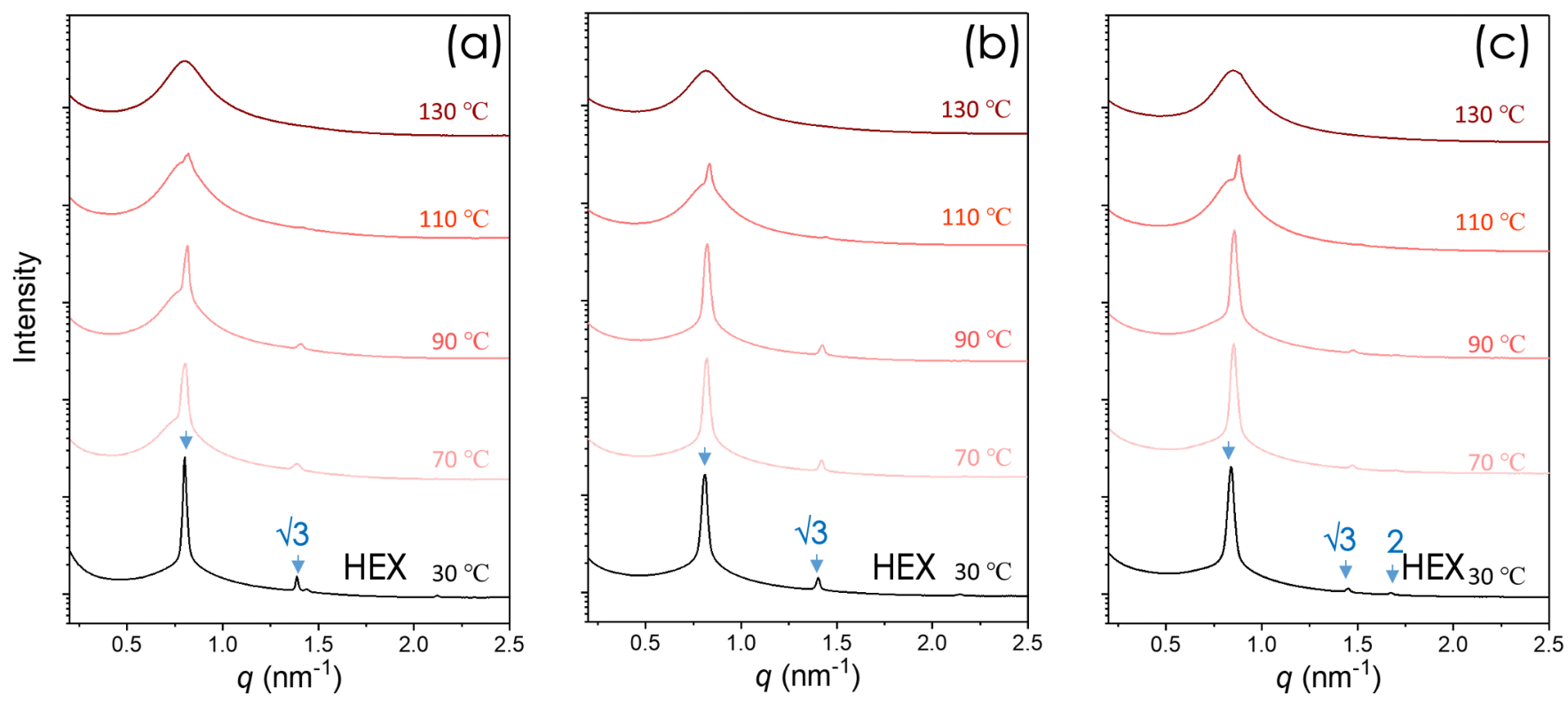

Figure S24. Temperature dependent SAXS profiles of the amphiphilic POSS chains: $p-C_{3} D(a), m-C_{3} D(b)$, and $o-\mathrm{C}_{3} \mathrm{D}(\mathrm{c})$.
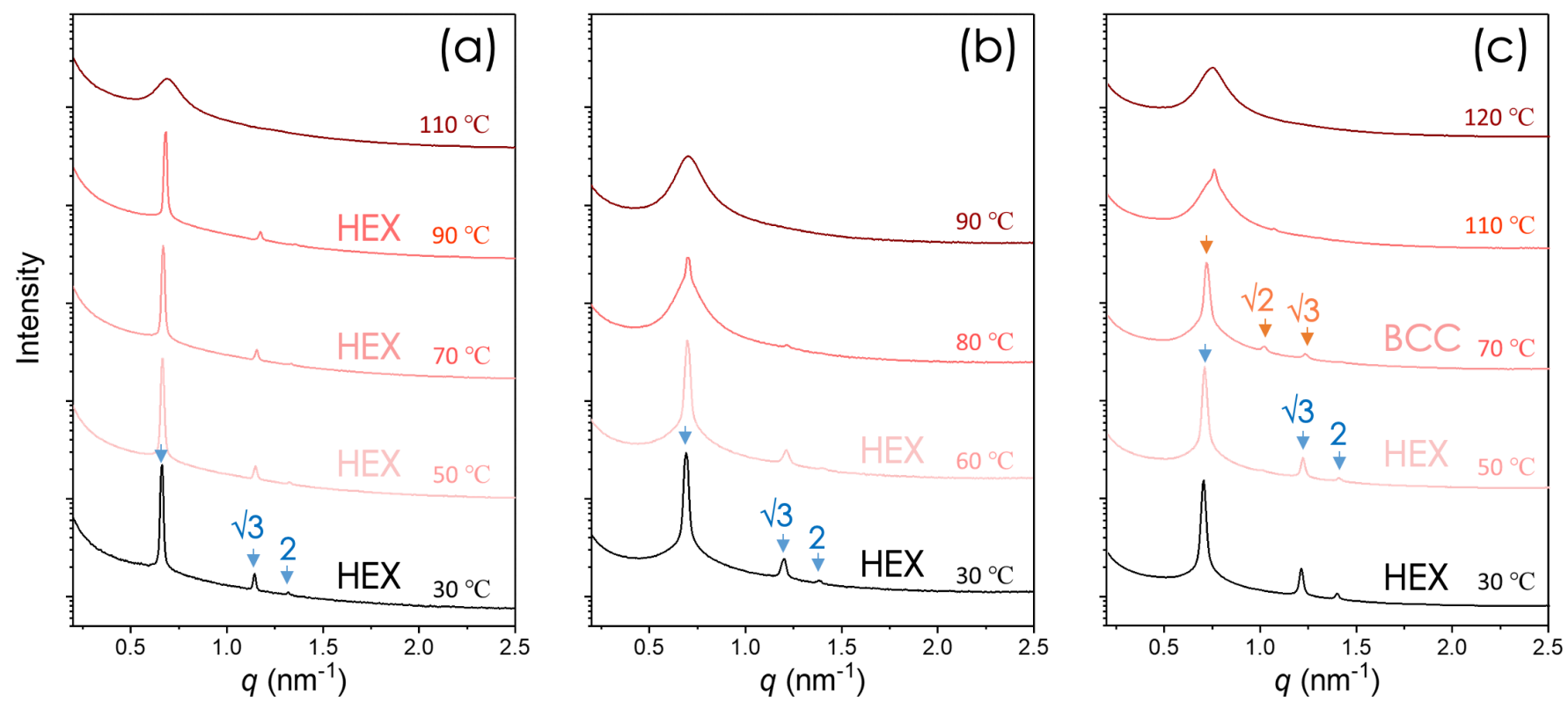

Figure S25. Temperature dependent SAXS profiles of the amphiphilic POSS chains: $p-{ }^{*} \mathrm{C}_{4} \mathrm{D}(\mathrm{a}), m-{ }^{*} \mathrm{C}_{4} \mathrm{D}$ (b), and $o-* \mathrm{C}_{4} \mathrm{D}(\mathrm{c})$. 
[1] Zhang, W.; Zhang, S. L.; Guo, Q. Y.; Lu, X. L.; Liu, Y. C.; Mao, J. L.; Wesdemiotis, C.; Li. T.; Li, Y. W.; Cheng, S. Z. D. Multilevel Manipulation of Supramolecular Structures of Giant Molecules via Macromolecular Composition and Sequence. ACS Macro Lett. 2018, 7, 635-640.

[2] Zhang, W.; Huang, M. J.; Su, H.; Zhang, S.; Yue, K.; Dong, X. H.; Li, X.; Liu, H.; Zhang, S.; Wesdemiotis, C.; Lotz, B.; Zhang, W. B.; Li, Y.; Cheng, S. Z. D. Toward Controlled Hierarchical Heterogeneities in Giant Molecules with Precisely Arranged Nano Building Blocks. ACS Cent. Sci. 2016, 2, 48-54. 